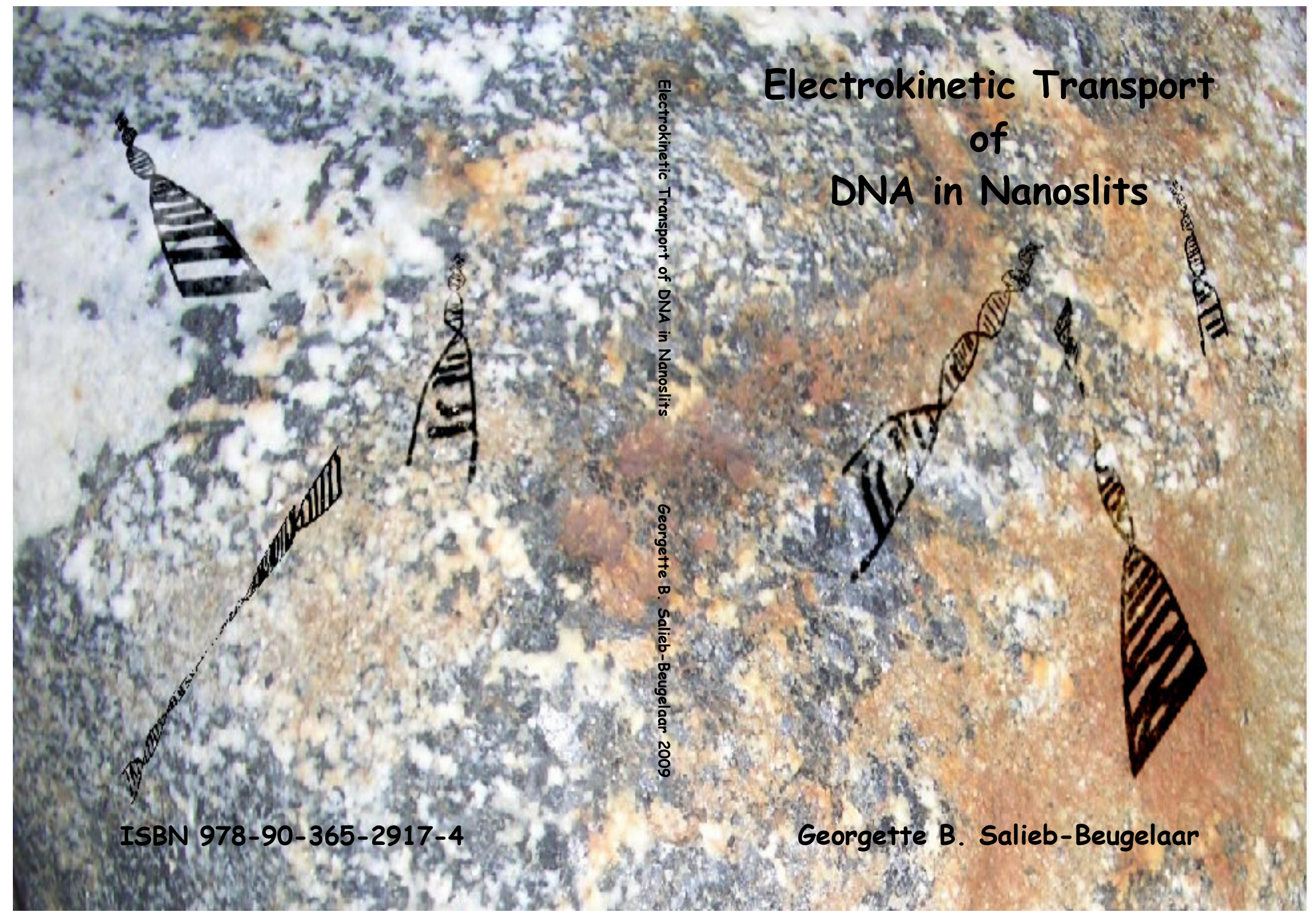


Filename: Cover Design For_WPS_Final

Directory: C: $\quad$ :Documents and Settings $\backslash$ Georgette.UTWENTEA174A94IDesktoplReady for printing

Template: C:LDocuments and SettingslGeorgette.UTWENTEA174A94\Application DatalMicrosoft \Templates\Normal.dot

Title:

Subject:

Author: Ljubomir Manola

Keywords:

Comments:

Creation Date: $\quad$ 10/28/2009 11:19:00 AM

Change Number: 2

Last Saved On: $\quad$ 10/28/2009 11:19:00 AM

Last Saved By: Georgette

Total Editing Time: 1 Minute

Last Printed On: $\quad$ 9/28/2009 4:33:00 PM

As of Last Complete Printing

Number of Pages: 1

Number of Words: 4 (approx.)

Number of Characters: 27 (approx.) 


\section{Electrokinetic Transport of \\ DNA in Nanoslits}


The described research has been carried out at the "Miniaturized Systems for Biomedical and Environmental Applications" group (BIOS) of the $\mathrm{MESA}^{+}$institute for nanotechnology at the University of Twente, Enschede, the Netherlands. The research was financially supported by the Dutch Ministry of Economic Affairs through a NanoNed grant (project: TSF7133).

Members of the committee:

Chairman prof. dr. A.J. Mouthaan University of Twente

Promotor prof. dr. ir. A. van den Berg University of Twente

Assistant promoter dr. J.C.T. Eijkel University of Twente

Members $\quad$ prof. dr. V. Subramaniam University of Twente prof. dr. J. Schmitz University of Twente dr. M.L. Bennink University of Twente prof. dr. P.S. Doyle MIT (US) prof. dr. A. Kristensen DTU Nanotech (DK)

\section{Euios $\frac{M E S A^{+}}{n}$ nano ned}

Title: $\quad$ ELECTROKINETIC TRANSPORT OF DNA IN NANOSLITS

Cover: $\quad$ Steingletschers (CH) Poem366 \& Grebeson Art, (C) 2009.

Author: $\quad$ Georgette B. Salieb-Beugelaar

ISBN: $\quad$ 978-90-365-2917-4

Printing: Wöhrmann Print Service, Zutphen, The Netherlands.
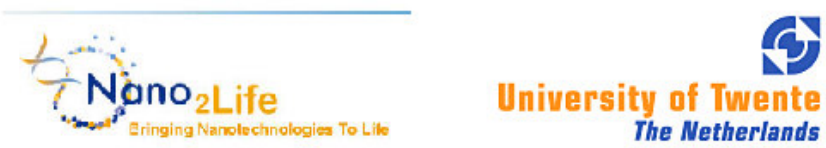


\title{
ELECTROKINETIC TRANSPORT OF DNA IN NANOSLITS
}

\author{
PROEFSCHRIFT
}

\author{
ter verkrijging van \\ de graad van doctor aan de Universiteit Twente \\ op gezag van de rector magnificus \\ prof. Dr. H. Brinksma \\ volgens het besluit van het College voor Promoties \\ in het openbaar te verdedigen \\ op vrijdag 30 oktober 2009 om 13:15
}

door

GEORGETTE BERNICE SALIEB-BEUGELAAR

geboren op 13 maart 1970

te 's Gravenhage 
Dit proefschrift is goedgekeurd door:

Promotor: $\quad$ prof. dr. A. van den Berg Assistent promotor: dr. J.C.T. Eijkel 
"Innovation is not the product of logical thought, although the result is tied to logical structure"

\section{Albert Einstein}



$\leftarrow$

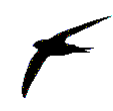

For my molecules of life....... 


\section{Contents}

1. Aim and Outline of Thesis $\quad 11$

$\begin{array}{lll}1.1 & \text { Introduction } & 12\end{array}$

$\begin{array}{ll}1.2 & \text { Electrokinetic transport of DNA in nanoslits } \\ 1.3\end{array}$

$\begin{array}{lll}1.3 & \text { Thesis outline } & 13\end{array}$

$\begin{array}{lll}1.4 & \text { References } & 14\end{array}$

2. Introduction $\quad \mathbf{1 5}$

$\begin{array}{ll}2.1 & 16\end{array}$

$\begin{array}{ll}2.2 \text { DNA structure } & 16\end{array}$

2.3 DNA in confinement 20

2.4 DNA electrophoresis 22

2.4.1 Free-solution electrophoresis 22

$\begin{array}{ll}\text { 2.4.2 Gel and capillary electrophoresis } & 24\end{array}$

2.4.2.1 Ogston sieving $\left(\mathrm{R}_{\mathrm{g}}<\mathrm{b}\right) \quad 24$

2.4.2.2 Biased reptation $\left(\mathrm{R}_{\mathrm{g}}>\mathrm{b}\right) \quad 25$

2.4.2.3 Entropic trapping $\left(\mathrm{R}_{\mathrm{g}} \sim \mathrm{b}\right) \quad 29$

2.4.3 Nanostructure electrophoresis 30

2.4.3.1 Free-solution electrophoresis in nanostructures 32

2.4.3.2 Electrostatic sieving, Ogston Sieving and entropic trapping 34

$\begin{array}{lll}\text { 2.4.3.3 Biased reptation } & 37\end{array}$

2.4.3.4 Electrophoresis in nanoslits and nanotubes 40

$\begin{array}{ll}2.5 & \text { Conclusions and perspectives }\end{array}$

2.6 References 44

3. DC Field Dependent DNA Mobility in 20 nm High Nanoslits 49

$\begin{array}{lll}3.1 & \text { Introduction } & 50\end{array}$

$\begin{array}{ll}3.2 & \text { Experimental protocol }\end{array}$

3.2.1 The nanoslit device 50

$\begin{array}{lll}3.2 .2 & \text { Electric setup } & 51\end{array}$

3.3 Results and discussion $\quad 51$

3.4 Conclusion 58

$\begin{array}{lll}3.5 & \text { References } & 59\end{array}$

4. AC/DC Field Dependent DNA mobility in 20 nm High Nanoslits 61

$\begin{array}{lll}4.1 & \text { Introduction } & 62\end{array}$

4.2 Experimental protocol 63

4.2.1 The nanoslit device $\quad 63$

$\begin{array}{lll}\text { 4.2.2 Electric setup } & 63\end{array}$

$\begin{array}{lll}4.3 & \text { Results and discussion } & 64\end{array}$

4.3.1.A Overall mobilities, $1 \mathrm{kHz}$ AC 66

4.3.1.B Overall mobilities, $100 \mathrm{~Hz} \mathrm{AC} \quad 67$ 
4.3.1.C Go-phase mobilities, $1 \mathrm{kHz} \quad 68$

4.3.1.D Go-phase mobilities, $100 \mathrm{~Hz} \quad 71$

4.3.1.E Summary of the obtained mobilities $(100 \mathrm{~Hz} ; 1 \mathrm{kHz})$

$\begin{array}{lll}\text { 4.3.2 Pathways and trapping sites } & 72\end{array}$

4.3.3 DNA orientation, increasing mobilities and biased reptation 73

$\begin{array}{lll}4.4 & \text { Conclusion } & 75\end{array}$

$\begin{array}{lll}4.5 & \text { References } & 76\end{array}$

5. DNA Mobility in 60 \& 120 nm High Nanoslits at Applied DC Fields $\quad 77$

$\begin{array}{lll}5.1 & \text { Introduction } & 78\end{array}$

$\begin{array}{lll}5.2 & \text { Experimental protocol } & 78\end{array}$

$\begin{array}{lll}5.2 .1 & \text { The nanoslit devices } & 78\end{array}$

$\begin{array}{ll}\text { 5.2.2 Electric setup } & 80\end{array}$

$\begin{array}{lll}5.2 .3 & \text { Measurement procedure } & 80\end{array}$

$\begin{array}{lll}5.3 & \text { Results and discussion } & 81\end{array}$

5.3.1 Mobility and nanoslit height $\quad 81$

$\begin{array}{lll}\text { 5.3.2 Pathways and trapping } & 82\end{array}$

$\begin{array}{lll}\text { 5.3.3 The force balance model } & 83\end{array}$

$\begin{array}{lll}\text { 5.3.4 Behavior at fields above } 1 \mathrm{MV} / \mathrm{m} & 85\end{array}$

5.3.5 Preliminary results with Litmus DNA 86

$\begin{array}{lll}5.4 & \text { Conclusion } & 87\end{array}$

$\begin{array}{lll}5.5 & \text { References } & 87\end{array}$

6. The Charge Patterned Nanoslit $\quad 89$

$\begin{array}{lll}6.1 & \text { Introduction } & 90\end{array}$

6.2 Device layout 91

6.3 Choice of material 92

6.4 Manufacturing process 92

$\begin{array}{lll}\text { 6.4.1 The top wafer } & 92\end{array}$

$\begin{array}{lll}\text { 6.4.2 The bottom wafer } & 96\end{array}$

6.4.3 Wafer bonding and dicing of the device 98

$\begin{array}{ll}\text { 6.4.4 Filling of the device } & 100\end{array}$

$\begin{array}{lll}6.5 & \text { Conclusions } & 100\end{array}$

$\begin{array}{lll}6.6 & \text { References } & 100\end{array}$

$\begin{array}{ll}\text { Appendix } & 101\end{array}$

$\begin{array}{ll}\text { 7. Conclusions and Prospectives } & 103\end{array}$

$\begin{array}{lll}7.1 & \text { Summary } & 104\end{array}$

$\begin{array}{lll}7.2 & \text { Outlook } & 105\end{array}$

7.2.1 DC field dependent DNA mobility in $20 \mathrm{~nm}$ high nanosli 106

7.2.2 AC/DC field dependent DNA mobility in $20 \mathrm{~nm}$ high nanoslits 106

7.2.3 DNA mobility in $60 \& 120 \mathrm{~nm}$ high nanoslits 107

$\begin{array}{lll}\text { 7.2.4 The charged-patterned nanoslit } & 107\end{array}$

Appendix 1: List of Abbreviations and Symbols 109 
Appendix 2: Single DNA Molecule Experiments

A.2.1 Introduction

A.2.2 The nanoslit device

114

A.2.3 The experimental setup

115

A.2.4 Electric potential measurement

117

A.2.5 Sample preparation

118

A.2.6 References

119

Samenvatting

121

Curriculum Vitae

List of publications

129

Acknowledgements / Dankwoord 
Filename: $\quad$ Front Page_For_WPS_Final

Directory: $\quad$ C:Documents and SettingsiGeorgette.UTWENTE-

A174A94lDesktoplDinsdag

Template:

C:IDocuments and SettingsiGeorgette.UTWENTE-

A174A94\Application DatalMicrosoft|Templates $\backslash$ Normal.dot

Title: 1

Subject:

Author:

Georgette

Keywords:

Comments:

Creation Date: $\quad$ 10/1/2009 3:48:00 PM

Change Number: $\quad 4$

Last Saved On: $\quad$ 10/1/2009 3:53:00 PM

Last Saved By: Georgette

Total Editing Time: 5 Minutes

Last Printed On: $\quad$ 10/1/2009 3:54:00 PM

As of Last Complete Printing

Number of Pages: 10

Number of Words: 753 (approx.)

Number of Characters: $\quad 4,295$ (approx.) 


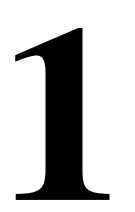

\section{Aim and Outline of Thesis}

In this chapter a brief introduction to the aims of the project, the investigation of the electrokinetic transport behaviour of DNA in nanoslits, is given. The advances of the use of nanofluidic devices and structures are briefly discussed. Finally an overview is presented of the subjects treated in the following chapters of this thesis. 


\subsection{Introduction}

The last three decades, the rapid development of micro Total Analysis Systems ( $\mu$ TAS) also called Lab on a Chip (LOC) systems, raised the perspective that these devices will be replacing larger equipment used in for example hospitals and industry ${ }^{1}$. Advantages of these systems are small sample volumes, fast analysis, high throughput, compability with portable and compact readout systems. The first commercial microfluidic platform for the analysis of DNA, RNA, proteins and cells was launched in $1994^{2}$. Recent developments in the field are for example microfluidic devices for size separation of DNA molecules by artificially created matrices ${ }^{3,4}$. These microfluidic devices are used to separate an entire ensemble of molecules. By introducing nanofluidics, single DNA molecules could be addressed into a LOC system. In the nanofluidic regime, many new phenomena were found, and investigation of single DNA molecules in this field can possibly lead to new LOC applications. An interesting example in this field is for example the stretching of a DNA molecule in a nanochannel as a result of the confinement ${ }^{5}$. The dimensions of confinement are very well controlled when using artificial nanostructures, which is a huge advantage when studying the fundamental physics and chemistry of DNA molecules. The mapping of restriction sites and protein binding sites along stretched DNA molecules was also presented in $2005^{6,7}$. The stretching could also be extremely interesting for the sequencing of a single DNA molecule or for the sequence mapping of DNA molecules by using fluorescent probes ${ }^{8}$. As can be concluded from this, nanofluidics is an extremely useful tool to develop a new generation of nanofluidic based LOC devices. Therefore, the aim of this thesis is to investigate the electrokinetic transport behaviour of single DNA molecules in nanoslits and to hopefully discover unknown properties of this molecule which could be used for the development of new (separation) LOC devices or to improve diagnostics.

\subsection{Electrokinetic transport of DNA in Nanoslits}

In the last decades, separation fundamentals of DNA have been investigated extensively in the world of gel electrophoresis. Excellent reviews in this field are for example those by Viovy ${ }^{9}$ or Slater ${ }^{10}$. The electrophoresis of DNA molecules, resulting in a separation of length is of high importance for many applied diagnostics as for example sequencing ${ }^{11,12}$ or genotyping ${ }^{13-15}$. For the last two mentioned techniques, based on the separation of length, still huge equipment is used. Therefore the development of "Lab on a Chip" (LOC) devices in this particular field is still desirable. Here we will focus on the fundamental behaviour of the DNA molecules when they are transported through the nanoslits by the use of an electrical field. The provided results will be compared with the main theories and models provided for gel electrophoresis.

This thesis describes the investigation of $\lambda$-DNA and Litmus DNA (XbaI digested) molecules electrokinetically transported through nanoslits. Chapter 3 of this project is the result of a collaboration between the University of Twente (UT), the University of Lund 
(UL, Sweden) and the University of Wildau (UW, Germany). The work at the UT was performed in the BIOS Lab-on-a-Chip group (part of the faculty of Electrical Engineering, Mathematics and Information Technology) of the Mesa ${ }^{+}$Institute for Nanotechnology. The work at the LU was performed at the Department of Physics/ Solid State Physics. Chapter 4 is the result of a collaboration between the University of Twente (UT) and the University of Barcelona (UB, Spain). The work at the UT was performed in the BIOS Lab-on-a-Chip group (part of the faculty of Electrical Engineering, Mathematics and Information Technology) of the $\mathrm{Mesa}^{+}$Institute for Nanotechnology. The work at the UB was performed at the Department of Electronics, Networking Research Center on Bioengineering, Biomaterials and Nanomedicine.

This project was funded by the Dutch Ministry of Economic Affairs through a NanoNed grant (TSF7133), Nano2Life Network of Excellence.

\subsection{Thesis outline}

Below the subject matter of each chapter of this thesis is presented.

In chapter 2 the important theories for free solution and gel electrophoresis are discussed. Examples of models discussed are biased reptation, Ogston sieving and entropic trapping. From this perspective, DNA separation using nanostructures is discussed and suggestions for future separation concepts are given.

Chapter 3 presents the results of experiments of $\lambda$-DNA in $20 \mathrm{~nm}$ high nanoslits when DC electrical fields are applied. A detailed description of the transport behaviour at various DC field strengths is given. Two types of movement were found, fluent and intermittent. Possible explanations for this behaviour are proposed. The mobility of Litmus DNA was also investigated. For both the mobility and the transport behaviour, differences between $\lambda$ DNA and Litmus DNA were found.

In chapter 4 the results of an investigation into the hypothesis of a dielectrophoretic trapping mechanism in the $20 \mathrm{~nm}$ high nanoslits are given. AC fields were superimposed onto DC fields to increase the local density of the electrical field lines. Also here the two types of movement (fluent and intermittent) were found. The observed transport behaviour of $\lambda$-DNA is described in detail and a possible explanation of the behaviour is given in terms of a biased reptation mechanism.

Chapter 5 describes the experimental results for the transport behaviour of $\lambda$-DNA in 60 $\mathrm{nm}$ and $120 \mathrm{~nm}$ deep nanoslits. Indications were found that the $\lambda$-DNA moves in a fundamentally different way in these slits than in the $20 \mathrm{~nm}$ slits. Intermittent movement of the $\lambda$-DNA molecules was only found in the $60 \mathrm{~nm}$ high nanoslits and for a small amount of the total number of $\lambda$-DNA molecules which were transported in a fluent way. 


\section{Chapter 1}

In chapter 6 the design and fabrication of a charged patterned nanoslit device is described. This device can be used to further investigate the transport behaviour of the DNA molecules in nanoslits by adjusting the double layer thickness and the surface charges.

Finally, in chapter 7 the conclusions of the work described in this thesis are summarized, Furthermore, several issues are suggested that can be useful for the improvement of future experiments with single DNA molecules in nanoslits. Next to this, suggestions for future research are given.

\subsection{References}

1. A. Manz, N. Graber, and H.M. Widmer, 1990. Sensors and Actuators B, 1, 1-6, 244248

2. D. Janasek, J. Franzke, and A. Manz, Nature, 2006, 442, 7101, 374-380.

3. O. Bakajin, T.A.J. Duke, J. Tegenfeldt, C.F. Chou, S.S. Chan, R.H. Austin, E.C. Cox, Analytical Chemistry, 2001, 73, 24, 6053-6056

4. J.Y. Han, J.P. Fu, R.B. Schoch, Lab on a Chip, 2008, 8, 1, 23-33.

5. J.O. Tegenfeldt, C. Prinz, H. Cao, S. Chou, W.W. Reisner, R. Riehn, Y.M. Wang, E.C. Cox, J.C. Sturm, P. Silberzan, R.H. Austin, 2004, PNAS, 2004, 101, 30, 10979 -10983.

6. R. Riehn, M. Lu, Y.-M. Wang, S.F. Lim, E.C. Cox, R.H. Austin, PNAS, 2005, 102, 29, 10012-10016.

7. Y.M. Wang, J.O. Tegenfeldt, W. Reisner, R. Riehn, X.-J. Guan, L. Guo, I. Golding, E.C. Cox, J. Sturm, R.H. Austin, PNAS, 2005, 102, 28, 9796-9801.

8. K. Jo, D.M. Dhingra, T. Odijk, J.J. de Pablo, M.D. Graham, R. Runnheim, D. Forrest, D.C. Schwartz, PNAS, 2007, 104, 8, 2673-2678.

9. J.L. Viovy, ReV. Mod. Phys., 2000, 72, 3, 813 - 872.

10. G. W. Slater, Electrophoresis 2002, 23, 3791-3816.

11. J. Guo, N. Xu, Z. Li, S. Zhang, J. Wu, D. H. Kim, M. Sano Marma, Q. Meng, H. Cao, X. Li, S. Shi, L. Yu, S. Kalachikov, J. J. Russo, N. J. Turro, J. Ju, Proc. Nat. Acad. Sci. USA, 2008, 105, 9145-9150

12. J. M. Prober, G. L. Trainor, R. J. Dam, F. W. Hobbs, C. W. Robertson, R. J. Zagursky, A. J. Cocuzza, M. A. Jensen, K. Baumeister, Science, 1987, 238, 4825, 336 - 341.

13. P. W. Reed, J. L. Davies, J. B. Copeman, S. T. Bennett, S. M. Palmer, L. E. Pritchard, S. C. L. Gough, Y. Kawaguchi, H. J. Cordell, K. M. Balfour, S. C. Jenkins, E. E. Powell, A. Vignal, J. A. Todd, Nat. Genet. 1994, 7, $390-395$.

14. A. Edwards, A. Civitello, H.A. Hammond, C.T. Caskey, Am. J Hum. Genet., 1991, 49, 746-756.

15. C.T. Caskey, A. Pizzuti ,Y.H. Fu, R.G. Fenwick, D.L. Nelson. Science, 1992, 256, 784 -789 . 
Filename: 1_Aim and outline of thesis_For WPS_Final

Directory: $\quad$ C:LDocuments and SettingsiGeorgette.UTWENTE-

A174A94\Desktop $\backslash$ Ready for printing $\backslash$ Dinsdag

Template:

C:Documents and Settings IGeorgette.UTWENTE-

A174A94\Application DatalMicrosoft|Templates $\backslash$ Normal.dot

Title: 1

Subject:

Author:

Georgette

Keywords:

Comments:

Creation Date: $\quad$ 9/29/2009 1:57:00 PM

Change Number: 2

Last Saved On: $\quad$ 9/29/2009 1:57:00 PM

Last Saved By: Georgette

Total Editing Time: 0 Minutes

Last Printed On: $\quad$ 9/29/2009 8:39:00 PM

As of Last Complete Printing

Number of Pages: 4

Number of Words: $\quad 1,296$ (approx.)

Number of Characters: 7,393 (approx.) 


\section{Introduction}

In this chapter, we recall the important theories developed for free-solution and gel electrophoresis of DNA such as the extended Ogston model, biased reptation and entropic trapping. From this perspective, suggestions for future concepts for fast DNA separation using nanostructures will be given.

This chapter was published in: G.B. Salieb-Beugelaar, K.D. Dorfman, A. Van den Berg and J.C.T. Eijkel, Lab Chip, 2009, 9, 2508-2523. 
Chapter 2

\subsection{Introduction}

Investigations in both nano- and microtechnology in the last decade have led to the development of new devices for analyzing and studying biomolecules such as DNA ${ }^{1-5}$. These devices have opened up a new world of possibilities for analyses by reducing the device size and sample consumption (lab-on-a-chip) ${ }^{6-10}$. In many instances, experiments have also shown that the dynamics of biomolecules in nanofluidic environments are markedly different from their behavior in macro- and microsystems. A general reason for this is the increased surface to volume ratio, leading to, amongst other factors, increased electrical and friction effects. For a macromolecule such as DNA, the transition towards nanoscale structures also leads to non-trivial confinement effects when the device length scales approach the pertinent length scales characterizing the DNA, such as its radius of gyration and persistence length (see section 2.2). The last several years have witnessed extensive experimental work on DNA transport in nano slits ${ }^{11-22}$, channels ${ }^{23-34}$, entropic traps and pillars ${ }^{35-45}$ and pores and other structures ${ }^{46-50}$. This chapter aims to explore the extent to which the available knowledge on the physicochemical properties of DNA in gels and the separation mechanisms underlying gel electrophoresis can be used to understand and interpret the results that have appeared thus far for DNA electrophoresis in nanostructures.

\subsection{DNA structure}

DNA or deoxyribonucleic acid is the molecule of life. It is a charged biopolymer (polyelectrolyte), built from only 4 different monomers which are placed in a very specific way (see figure 2-1). The monomers are called the nucleotides and they are constructed from the bases adenine $(A)$, thymine $(T)$, guanine $(G)$ and cytosine $(C)$, covalently linked to a deoxyribose sugar and a phosphate group. To form a DNA strand, the nucleotides are covalently linked via phosphodiester bonds. In the doublestranded DNA molecule depicted in figure 2-1, the two homologous strands coupled via hydrogen bonds in a complementary way and form the double helix structure originally described by Watson and Crick ${ }^{51}$. In the standard Watson-Crick base pairing, the adenine of one strand forms two hydrogen bonds with the thymine of the homologous strand, while guanine and cytosine pair via three hydrogen bonds. In the common helical form, known as B-DNA, the spacing between base pairs is $\sim 3.4 \AA$ in length. In the standard dogma, the sequence of basepairs codes for all necessary information to assemble the parts of a living organism, from bacteria flagella to the color of human eyes.

In this chapter, DNA molecules are assumed to be double-stranded unless specifically mentioned otherwise. In the double-stranded form, the bases are on the inside of the helix and "hidden" from the surrounding ionic environment. Hence, it is reasonable to treat long double stranded DNA as a homogeneous semi-flexible biopolymer consisting of $N$ 


\section{Introduction}

monomers of length $d$ (one basepair) and a total contour length $L=N d$ (contour length). The semi-flexible nature of DNA is quantified by the persistence length $p$, which is much larger than the size of one monomer and captures the stiffness of the molecule. A typical value for the persistence length of DNA is about $50 \mathrm{~nm}$ (about 150 base pairs). The apparent persistence length depends on a number of factors, including the ionic strength of the solution and any electrostatic forces (resulting from phosphate group repulsion) inside the molecule chain ${ }^{52-54}$. In many electrophoretic applications, the DNA is also stained with an intercalating dye for visualization, which can also influence its mechanical properties.

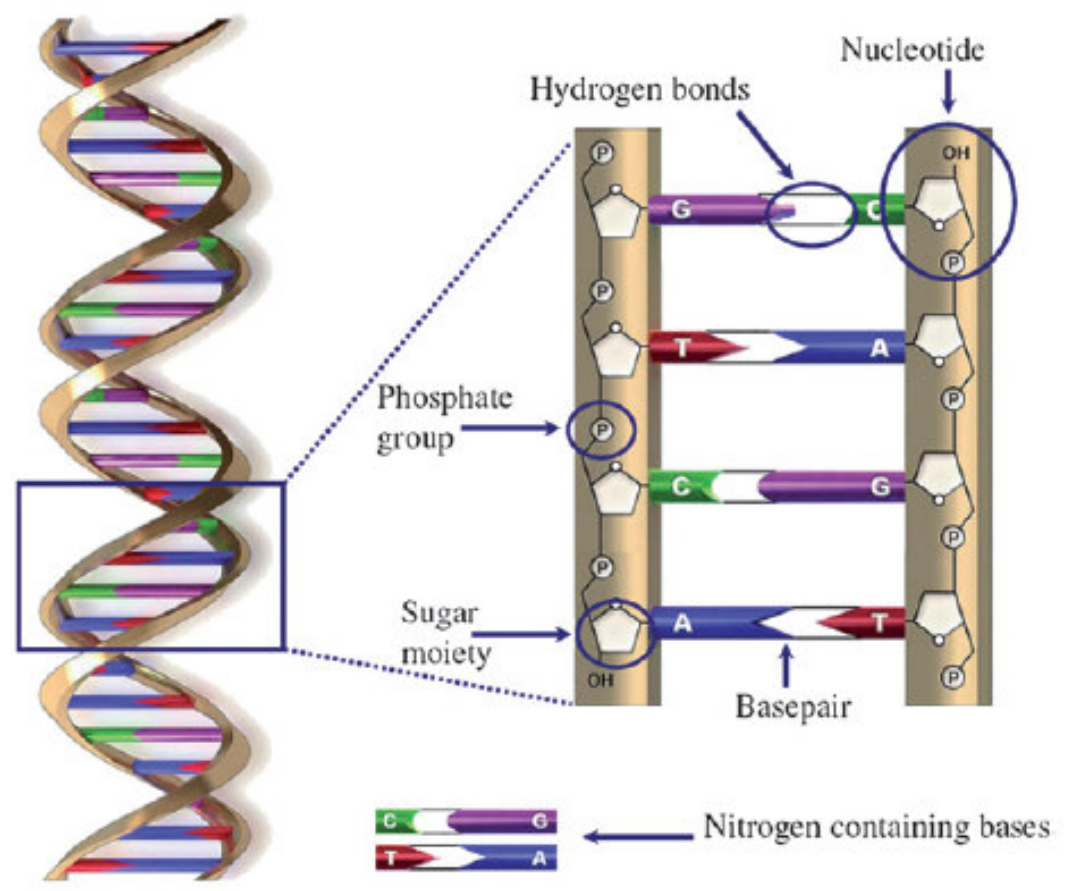

Figure 2-1. The double helix structure of DNA. It is constructed of four different building blocks called nucleotides. Each nucleotide is covalently linked with its sugar moiety to the phosphate group of its adjacent nucleotide. Nucleotides can also form basepairs (bp) via hydrogen bonding. Each adenosine is coupled with thymine and each cytosine with guanine.

When describing the dynamics of DNA molecules, the persistence length is often replaced by the Kuhn length ${ }^{55-58}$ to avoid carrying through the numerical prefactor,

$$
l_{\text {Kuhn }}=2 p
$$

In the simplest elastic model of DNA, each Kuhn segment is modeled as freely joined with the next segment, and depending on its length the biopolymer can be divided into $N$ Kuhn segments, 
Chapter 2

$$
N_{\text {Kuhn }}=\frac{L}{l_{\text {Kuhn }}}
$$

When the contour length is much larger than the Kuhn length $\left(l_{\text {Kuhn }}<<L\right)$, the molecule can be described by a random walk of Kuhn segments.

The charge of the biopolymer is also a very important parameter. In this chapter, we assume that the charge density $\sigma$ of the DNA molecule is uniform along the molecule. By using the Kuhn length as a base length scale, it will prove useful later to introduce the charge per Kuhn length,

$$
q_{\text {Kuhn }}=\frac{\sigma}{l_{\text {Kuhn }}}
$$

To describe the motion of DNA through a surrounding liquid, we will use the friction per Kuhn length,

$$
\xi_{\text {Kuhn }} \approx \eta l_{\text {Kuhn }}
$$

where $\eta$ is the viscosity of the solvent surrounding the molecule.

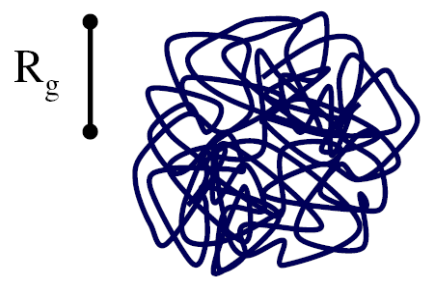

Figure 2-2. The 3D blob conformation of a DNA molecule in free solution. Picture after reference 59.

Imagine we extract DNA out of a blood sample and put the molecules into a buffer. What will happen to the molecules? Of course this will depend on the solution ${ }^{60}$. A commonly used buffer is the $1 \mathrm{x}$ Tris Borate Sodium EDTA buffer (TBE). When immersed in such a buffer, the DNA will now form a 3D blob in free solution (see figure 2-2). The radius of gyration $R_{g}$, which describes the average dimension of the molecule, depends strongly on the stiffness of the molecule. For a random walk of Kuhn segments without excluded volume, the radius of gyration is given by.

$$
R_{g}=l_{K u h n} N_{K u h n}^{1 / 2}
$$




\section{Introduction}

However, for very long DNA chains, the excluded volume interactions become important and introduce an extra repulsion that swells the chain. This leads to a scaling $R_{g} \sim N^{v}$. The excluded volume parameter (or Flory exponent) $v$ characterizes the DNA-solvent interactions and thus depends on the properties of both the molecule and the solvent ${ }^{61,62}$. For a good solvent, $v=3 / 5$.

As already discussed above, DNA molecules have negative phosphate groups attached to their backbone which provide electrostatic repulsion. The length scale characterizing when electrostatic interactions between the charges along the backbone are equal to thermal energy is the Bjerrum length ${ }^{63}$,

$$
l_{B}=\frac{e^{2}}{4 \pi \varepsilon_{b} \varepsilon_{0} k_{B} T}
$$

where $e$ is the elementary charge, $\varepsilon_{\mathrm{b}}$ the dielectric constant of the medium, $\varepsilon_{\mathrm{o}}$ the permittivity of vacuum and $k_{B} T$ is the Boltzmann factor.

In water at room temperature, the Bjerrum length is around $7 \AA$. Highly charged polymers such as DNA have their charges spaced much more closely than the Bjerrum length. As noted above, the electrostatic repulsion leads to an increase in the persistence length. However, when the charge spacing on the chain is less than the Bjerrum length, counterions are hypothesized to "condense" onto the chain to reduce the effective charge spacing on the backbone to the Bjerrum length, a phenomenon called Manning condensation. For further reading see also ref. 54 and 63-67.

The conformation of the molecule in free solution depends on the ionic strength of the buffer and the kind of counterions present. The negatively charged DNA molecule will attract oppositely charged ions, whereas negative charged ions are repulsed. In the fluid proximate to the chain, the counterion concentration then decays exponentially. The electrostatic potential in equilibrium may be found by using the general solution of the Poisson-Boltzmann equation, which is also called the Gouy-Chapman model ${ }^{68}$. The characteristic length scale for the decay of the charge layer around the DNA (or any charged surface) is called the Debye layer. The thickness of the layer is given by

$$
\lambda_{D}=\sqrt{\frac{\varepsilon_{0} \varepsilon_{b} k_{B} T}{2 e^{2} I}}
$$

The Debye length can be easily change in experiments by changing the ionic strength of the fluid, which is defined as

$$
I=\frac{1}{2} \sum_{i} z_{i}^{2} c_{i}
$$


Chapter 2

where $z_{i}$ the valence of ion $i$ and $c_{i}$ the concentration of ion $\mathrm{i}$. At an ionic strength of $10 \mathrm{mM}$ the Debye length is $\sim 1 \mathrm{~nm}$ and at an ionic strength of $100 \mu \mathrm{M}$ the Debye length is $\sim 10$ $\mathrm{nm}$. In most investigations mentioned in this chapter, a typical Debye layer thickness is between $1-3 \mathrm{~nm}$. The thickness of the Debye length therefore is thin when compared to the dimensions of the DNA molecule, unless specifically mentioned. The properties of this layer will be discussed further in the nanostructure section.

\subsection{DNA in confinement}

The change in the conformation of the DNA is a function of the degree of confinement. In the absence of any confinement, we model the DNA as the 3D blob shape as depicted in figure 2-2. When this molecule enters a nanostructure, it has to deform when the dimensions are smaller than the twice the radius of gyration. A nanostructure can be a capillary, nano channel, slit or a pore matrix (gel). In this chapter we use the word nanoslit when the confinement is strong in one dimension and in the other dimensions there is no or weak confinement, and nanochannel when the confinement is strong in two dimensions. For pore matrixes, we will assume that the confinement is tube-like. The precise characteristics of this tube-like confinement in a gel will be further explained in the gel electrophoresis (biased reptation) part of this chapter.

For capillaries, nanoslits and nanochannels, two theories are frequently invoked to describe the static conformation of confined DNA: the deflection (Odijk) model and the blob (de Gennes) model. The choice of model depends on the degree of confinement. Let us consider first the "Odijk" regime, where the DNA molecule is confined in a capillary or tube of whose diameter is smaller than the persistence length of DNA. The Odijk deflection theory ${ }^{69}$ assumes that, when the contour of the chain reaches the wall, it will be deflected and change its orientation (see also figure 2-3). The length scale characterizing the projection of the chain in the direction parallel to the channel walls is the deflection length

$$
l_{\text {deflection }} \sim\left(D^{2} p\right)^{1 / 3}
$$

In most circumstances, nanochannels do not possess a single length-scale characterizing their confinement. From a practical standpoint, the different lengths are a result of the fabrication process; the lithographic patterning of the substrate leads to a width $D_{\text {width }}$ that is, in general, different than the channel height $D_{\text {height }}$ obtained when the pattern is transferred into the substrate. In this case, the diameter D is replaced by the geometric averaged diameter

$$
D_{a v}=\sqrt{D_{\text {width }} D_{\text {height }}}
$$




\section{Introduction}

This deflection length is smaller than the persistence length and can be assumed as a rigid rod or segment. The complete molecule can now be seen as a semiflexible chain with $L /$ $l_{\text {deflection }}$ segments.
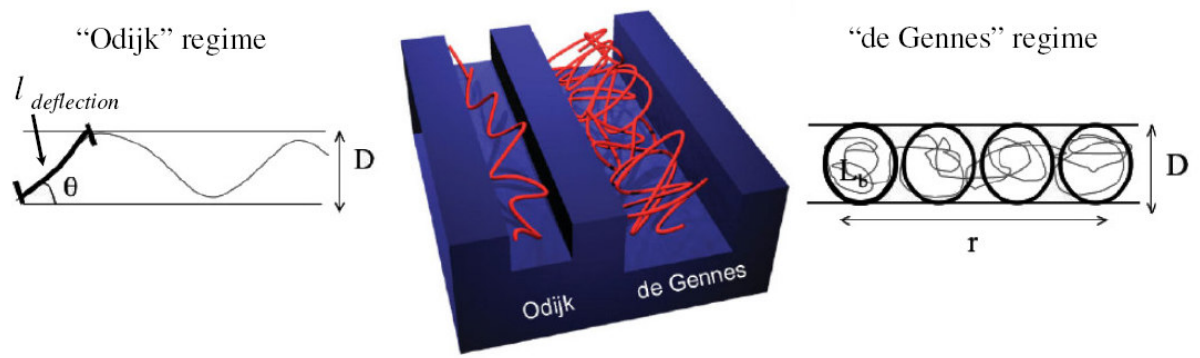

Figure 2-3. DNA molecules in two different regimes of confinement. In the Odijk regime, the DNA molecule is confined in a capillary or nanoslit whose characteristic cross-sectional length scale $D$ is smaller than the DNA persistence length $p$. In this way, the chain is deflecting off the walls and changing its direction. In Odijk theory the deflection length $l_{\text {deflection }}$ is introduced, which can be assumed as a rigid rod or segment ${ }^{69}$. In the de Gennes regime, the diameter of the capillary or nanoslit is larger than the persistence length. The molecule can now be defined as a series of blobs. As a result of the repulsion between the blobs and the confinement, the DNA molecule is extended to a length $r^{70}$. Reprinted with permission from ref. 1 and 26 .

The second theory applies to less stringent confinements than the Odijk theory. It is called the "blob" theory and was introduced by de Gennes in $1976{ }^{70}$ to describe the effect of confinement on polymers. This theory was later extended to describe polymers such as DNA in confinement ${ }^{71,72}$. The molecule can be considered as a chain of blobs. Each blob has a contour length $L_{b}$. As a result of the confinement of the DNA molecules and the repulsion between the blobs, the chain is extended to a distance

$$
r \approx L \sqrt{\frac{w_{e f f} p}{D^{2}}}
$$

where $\mathrm{w}_{\text {eff }}$ the effective width of the molecule and D the diameter of the capillary or nanochannel. The effective width is the intrinsic width, which includes the electrostatic contribution. Both theories provide different scaling of the dimensions, diffusion and relaxation times of polymers. For further reading, see Hsieh and Doyle ${ }^{73}$ and Odijk ${ }^{74}$ who clearly explain the models and their scaling. The parts of the "blob" theory which are relevant will be further discussed in the DNA electrophoresis section of this chapter. Having outlined the static conformations of DNA in free solution and confinement, let us now consider the electrophoresis of DNA, starting with free flow electrophoresis and continuing with gel and nanostructure electrophoresis 
Chapter 2

\subsection{DNA electrophoresis}

As discussed in the previous section, DNA molecules can be confined in several ways. In general, DNA electrophoresis requires some kind of confining geometry. As we will discuss in the following section, most DNA cannot be separated in free-solution so its interactions with the confining structure (either a gel, capillary or a nanostructure) are required in order to make electrophoretic mobility a function of molecular weight. From a practical standpoint, the gel, the capillary or nanostructure also suppresses backflow during the electrophoretic motion, which is equally important in obtaining a sharp separation.

\subsubsection{Free-solution electrophoresis}

Is it possible to separate DNA by size in free solution? As seen in figure 2-4, the mobility of short fragments is indeed a function of molecular weight, but the mobility eventually reaches a plateau at high molecular weights. The location of the plateau is a function of the buffer; for this particular experiment, the mobility becomes independent of molecular weight at $\sim 400 \mathrm{bp}$.

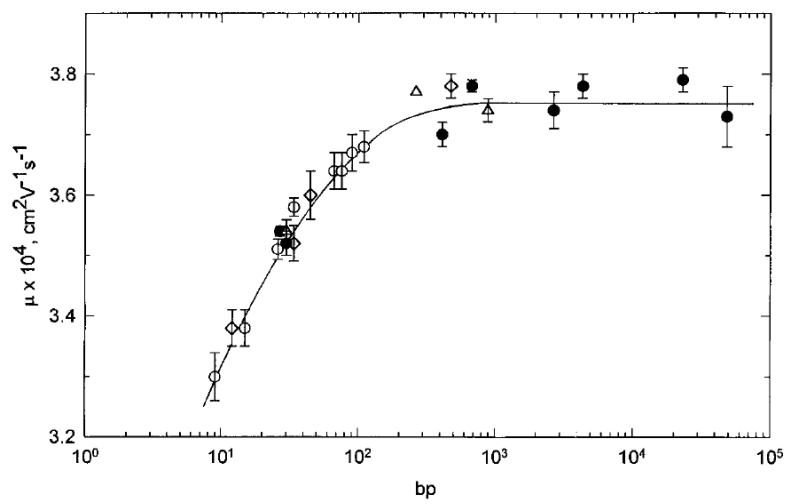

Figure 2-4. The free solution electrophoretic mobility of DNA molecules in TAE buffer. As can be clearly seen in this graph, the DNA molecules reach a plateau phase around a size of $400 \mathrm{bp}$. The plateau electrophoretic mobility here is $\sim 3.75 \times 10^{4} \mathrm{~cm}^{2} \mathrm{~V}^{-1} \mathrm{~s}^{-1}$. Thus, larger fragments cannot be separated from each other. Reprinted with permission from ref. 60.

The different regimes of free-solution DNA electrophoresis can be classified by the relative magnitudes of the persistence length $p$, the Debye length $\lambda_{\mathrm{D}}$, and the radius of gyration of the chain, $R_{g}$, following the work of Desruisseaux et al. ${ }^{75}$ (see figure 2-5). The molecularweight dependent regime (A) occurs in the limit $p>\lambda_{\mathrm{D}}>R_{g}$. 


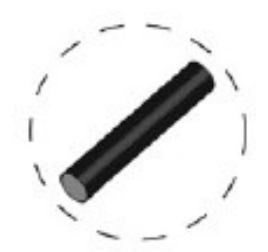

$$
p>\lambda_{D}>R_{g}
$$

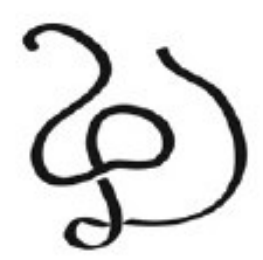

$$
R_{g}>p>\lambda_{D}
$$

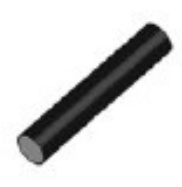

$$
p>R_{g}>\lambda_{D}
$$

Figure 2-5 The three different regimes of (bio) polymers in free solution. In regime A, the molecule can be treated as a charged rod in a sphere. Here, $p>\lambda_{\mathrm{D}}>R_{\mathrm{g}}$. Here separation is possible. In regime $\mathrm{B}$, the molecule can be seen as a random coil, $R_{\mathrm{g}}>p>\lambda_{\mathrm{D}}$. In regime $\mathrm{C}$, the molecule is seen as a long rod, where $p>R_{\mathrm{g}}>\mathrm{D}$. In regimes $\mathrm{B}$ and $\mathrm{C}$, no separation is possible. Image after ref. 75 .

In this case, we treat the DNA molecule as a rigid, charged rod. The radius of gyration is thus the size of the sphere swept out by the possible angular orientations of the rod due to rotational diffusion. In the large Debye limit depicted in figure 2-5A, the counterions are far from the chain and do not interact hydrodynamically with the rod. The resulting electrophoretic mobility is

$$
\mu_{0}=\frac{\sigma_{B}}{3 \pi \eta} \ln \left(\frac{L}{d}\right)
$$

for a charge density per Bjerrum length $\left(\mathrm{e}_{\mathrm{e}} / l_{\mathrm{B}}\right)$ of ${\sigma_{\mathrm{B}}}^{75}$. In regime $(\mathrm{A})$, DNA molecules can be separated by length when the molecules are small (so that $R_{g}$ small) and/or at very low salt concentrations (so that $\lambda_{D}$ is large).

The mobility becomes independent of length in both regimes (B) and (C) in figure 2-5. Once the radius of gyration becomes larger than the Debye length, we need to consider the interactions between the counterions and the chain. When an electric field is applied, the DNA will be attracted to the cathode and the counter ions will be attracted to the opposite direction, towards the anode. The hydrodynamic interactions are screened over the Debye length, so the mobility of the chain is equivalent to the mobility of a single segment of the chain. As shown by Manning, the relevant length scale for the hydrodynamics is the charge spacing $A$ and we thus have

$$
\mu_{0}=\frac{\sigma}{3 \pi \eta} \ln \left(\frac{\lambda_{D}}{A}\right)
$$

where $\sigma$ is the charge density after Manning condensation ${ }^{76}$. This hydrodynamic behavior is also called freely draining, and the conclusion is that it is impossible to separate DNA in free solution unless the DNA molecule is smaller than the Debye length. 
Chapter 2

According to the above mentioned regimes, it is clear that separation of biopolymers in free solution is very difficult. In regime A, only short DNA can be separated, and even then the salt concentration needs to be very low. In regime B and C, the DNA molecules act as freely-draining polyelectrolytes whose charge to friction ratio is not a function of the length of a molecule. For this reason, gel matrices are used to separate DNA molecules, which will be discussed in the following section.

\subsubsection{Gel and capillary electrophoresis}

Gels and polymer matrices are commonly used to analyze DNA molecules, for example in diagnostics. One of the most common gels is agarose, a polysaccharide of zero net charge. Agarose is a physical gel; agarose can be dissolved in buffer by heating and the gel is formed upon cooling. The pore sizes of agarose are hundreds of nanometers and thus appropriate for separating DNA from the $100 \mathrm{bp}$ up to tens of kilobasepairs. For separating smaller double-stranded DNA (20-1000 bp) and single-stranded DNA, polyacrylamide gels are more suitable. Polyacrylamide gels are chemically crosslinked and pore sizes down to a few nanometers can be achieved by controlling the chemistry used in the gel preparation. It is generally difficult to load gels into capillaries, so entangled polymer matrices are often employed for capillary electrophoresis. Common matrices include linear polyacrylamide and commercial formulations such as POP (Performance Optimized Polymer). In general, capillary electrophoresis is also used for separating shorter double-stranded DNA and single-stranded DNA, although long DNA can be separated by field-inversion capillary electrophoresis.

Useful reviews of DNA electrophoresis were written by Heller et al. ${ }^{77}$, Viovy ${ }^{78}$, Slater et al. ${ }^{79}$ and Deen ${ }^{80}$. When modeling DNA electrophoresis in a gel or entangled polymer network, it is common to model the sieving matrix as a maze or network of pores of size $b$. This is not strictly correct for entangled polymer networks due to reptation of the neutral polymer chains; the translation of theories for gels ${ }^{81,82}$ directly to entangled polymers has had many successes ${ }^{83-85}$ and some failures ${ }^{86}$. The ratio of pore size and the dimensions of the DNA molecule are very important for the way the DNA molecules migrates through the gel, which will be the subject of the following sections. We can distinguish three main separation regimes: (i) the Ogston sieving regime, where the pore size $b$ is larger than the radius of gyration $R_{g}$; (ii) the biased reptation regime, where $b$ is smaller than $R_{g}$; and (iii) the entropic trapping regime, where $b$ is around $R_{g}$. In the following part these three different regimes of separation will be briefly explained.

\subsubsection{Ogston sieving $\left(R_{\mathrm{g}}<\mathrm{b}\right)$}

Here the radius of gyration of the DNA molecules is smaller than the nominal pore size. In general, the DNA migrates through the network of pores without perturbation of their conformation. This is, of course, a somewhat simplified explanation. 
Real gels are inhomogeneous and possess a range of pore sizes, some of which could be smaller than the radius of gyration of the DNA. As we will see in the nanostructures section, fabrication allows one to create systems with uniform and well-defined pore structures that correspond directly to these Ogston sieving length scales ${ }^{35}$. The standard model for electrophoresis in this regime is normally called Ogston sieving ${ }^{87,88}$, although the model used for the interpretation of the mobility is more properly referred to as the extended Ogston model or the ORMC model ${ }^{87,89,90}$. The OMRC model states that, for a particle of size $R$ in a gel of concentration $c$, the mobility is equal to the fractional volume available to the particle,

$$
\frac{\mu}{\mu_{0}}=f(R, c)
$$

The "Ogston" part of the model refers to Ogston's ${ }^{87}$ calculation of the fractional volume for a point-sized particle in a random dispersion of fibers, which can be used with Eq. (214) to furnish the mobility

$$
\frac{\mu(R, c)}{\mu(R, 0)}=\exp [-K(R) c]
$$

where $K(\mathrm{R})=R+R_{f i b e r}$ and $R_{f i b e r}$ is a correction for the finite size of the gel fibers. This extended model has been very successful in the prediction of gel electrophoresis result through the use of Ferguson plots of $\ln \mu$ against the gel concentration. However, this model should only be used in low-field predictions. For medium to high field electrophoresis, the field dependent mobility shifts, which cannot be explained by this model. There are a number of phenomena missing in the ORMC model, such as the role of gel disorder, percolating pathways and dead-ends. Slater and co-workers ${ }^{91-100}$ have called into question the fundamental validity of the ORMC model for describing gel electrophoresis in the limit $\mathrm{R}_{\mathrm{g}}<\mathrm{b}$.

\subsubsection{Biased reptation $\left(R_{g}>b\right)$}

When the radius of gyration of the DNA is larger than the nominal pore size of the gel, the DNA must uncoil in order to enter the pore space. The DNA moves through the gel like a snake in a tube (the "reptation" part) and the electrical force causes preferential motion in the direction of the electric field (the "biased" part). The basic idea of biased reptation, which builds upon de Gennes' ${ }^{101}$ concept of reptation in polymer melts, was originally proposed by Lerman \& Frisch ${ }^{102}$ and Lumpkin \& Zimm ${ }^{103}$. Within the pores of the gel, the DNA is assumed to be in a Gaussian conformation and each pore contains $\left(b / l_{\text {Kuhn }}\right)^{2}$ Kuhn segments. As a result, a DNA molecule consisting of $N_{\text {Kuhn }}$ Kuhn segments can be envisioned as a series of $N_{b l o b}=N_{\text {Kuhn }}\left(l_{\text {Kuhn }} / b\right)^{2}$ blobs. As indicated in figure 2-6, as the 


\section{Chapter 2}

DNA reptates through the reptation tube, the blob at the rear of the chain is "destroyed" and a new blob is "created" at the front of the chain. In the absence of the field, there is an equal probability of moving to the left or right in figure 2-6 ${ }^{101}$. In the presence of an electric field of magnitude $E$, the reptative motion is biased so that the chain tends to form the new segment in the direction of the electric field.

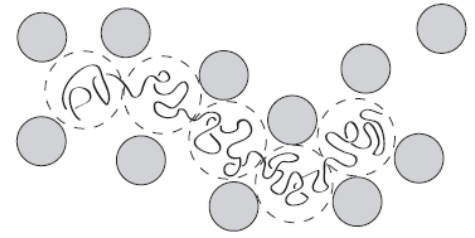

(a)

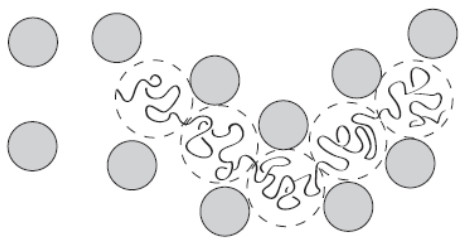

(b)

Figure 2-6 Schematic illustration of biased reptation from configuration (a) to configuration (b). At the rear of the chain, one blob is "destroyed" whereas at the front of the chain a new blob is "created".

In order for the DNA to stay inside the reptation tube, the electric field must be weak enough so that the entropic penalty for forming the hernia depicted in figure 2-7 is large compared to the decrease in enthalpy.

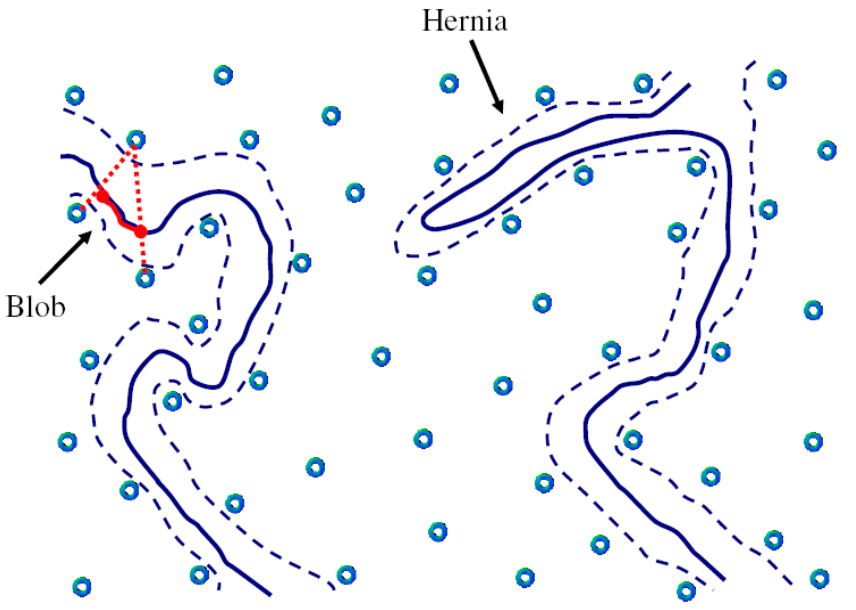

Figure 2-7 The tube model of DNA transport in a gel. The solid line represents the molecule, whereas the dotted lines are representing the tube. In A, the molecule moves through a network of pores. The part of the chain which is in the pore (red part) is called blob. In B, the molecule formed a loop or hernia. Picture after ref. 78.

Using a local force picture, the electric field strength must satisfy $E \ll k_{B} T / q_{\text {blob }} b$, where $k_{B} T$ is the Boltzmann factor and $q_{\mathrm{blob}}$ is the effective charge of one blob ${ }^{78}$. While the latter 


\section{Introduction}

provides a reasonable estimate for the upper bound of reptation in agarose gels, a more detailed model has suggested that biased reptation is inherently unstable and that hernias should form even at very weak fields ${ }^{104}$. The electrophoretic mobility of the chain is determined by a force balance on the chain of blobs. The electrical force $\mathrm{F}_{\mathrm{el}}$, acting on the chain is

$$
F_{e l}=Q E h_{x} / L
$$

where $Q$ is the effective charge and $L$ the contour length of the chain. The parameter $h_{x}$, which will play a key role shortly, is the net projection of the string of blobs in the direction of the electric field. The electrical force is opposed by a frictional force $\mathrm{F}$,

$$
F=v_{\text {tube }} \eta L
$$

where $v_{\text {tube }}$ is the curvilinear velocity of the DNA as it moves through the reptation tube and $\eta$ is the viscosity of the fluid. Balancing these two forces,

$$
v_{\text {tube }}=\mu_{0} E h_{x} / L
$$

and

$$
\mu_{0}=Q / \eta L
$$

where $\mu_{0}$ is the free-solution mobility. Thus, it takes a time $L / v_{\text {tube }}$ for the DNA to reptate a distance $h_{x}$ in the direction of the electric field. (This time is commonly referred to as the tube renewal time.) At this point, the DNA will have formed a new tube with a different projection $h_{\mathrm{x}}$. After the tube renewal process has repeated numerous times, the electrophoretic mobility of the reptating chain is given by

$$
\mu / \mu_{0}=\left\langle h_{x}^{2} / L^{2}\right\rangle
$$

where the brackets indicate an average over all of the reptation tubes.

Based on this simple model, one arrives at two different regimes for biased reptation. When the field is very weak, the string of blobs is itself a random walk. As a result, the characteristic size of the string of blobs is $h_{x} \sim L^{1 / 2}$ and the electrophoretic mobility scales like $L^{-1}$. This first regime, where a separation is possible, is called biased reptation without orientation. When the field is stronger, the string of blobs tends to be oriented in the direction of the electric field. In this case, the projection is $h_{x} \sim L$ and the mobility becomes independent of molecular weight. This second regime, where no separation is possible, is called biased reptation with orientation. 
Chapter 2

The above model of biased reptation has two shortcomings: (i) The chain is always assumed to move "head-first" in the direction of the electric field. While the reptation is biased towards that direction, there should also be some diffusive motion towards the back of the reptation tube. Adding backwards jumps into the biased reptation model ${ }^{105}$ leads to non-trivial results as the field strength increases, since backward jumps lead to J-shapes that trap the chain ${ }^{106}$; (ii) The biased reptation model predicts that the mobility scales like $E^{2}$ in the orientated regime. However, simulations of reptation-like motion by more complex models ${ }^{107-109}$ indicated that the mobility actually scales linearly with $E$. The origin of the linear scaling is a branched structure formed by the front end of the chain as it explores the different pores of the gel while dragging the rest of the chain behind it ${ }^{108}$. By incorporating this idea in a "biased reptation with fluctuations" model ${ }^{110}$, it is possible to capture both the $\mu \sim L^{-1}$ scaling in the unoriented regime and the $\mu \sim E$ scaling in the oriented regime. Moreover, the original biased reptation model is recovered when the pore size of the gel is close to the Kuhn length of the DNA ${ }^{111}$. The predictions of the biased reptation with fluctuations model agree well with experiments in a range of conditions ${ }^{112-}$ 115

The biased reptation model makes predictions about the electrophoretic mobility in two different regimes. There have also been a number of efforts to develop interpolation formulas that capture the behavior over the full range of molecular weights and electric fields. For example, Viovy ${ }^{116}$ proposed an interpolation formula

$$
\frac{\mu}{\mu_{0}}=\left\{\left[\frac{(b / l)^{2}}{3 N_{K u h n}}\right]^{2}+\left[\frac{2 \varepsilon_{k}(b / l)^{2}}{5+2 \alpha \varepsilon_{k}(b / l)^{2}}\right]^{2}\right\}^{1 / 2}
$$

where $\varepsilon_{k}=\eta l^{2} \mu_{0} \mathrm{E} / \mathrm{k}_{\mathrm{B}} \mathrm{T}$ and $\alpha$ is an $\mathrm{O}(1)$ parameter that captures $\mu_{0} / \mu$ in the large field limit ${ }^{117}$. A similar interpolation formula was also developed from the repton model ${ }^{109}$. More recently, Van Winkle et al. ${ }^{118}$ proposed a simple interpolation formula that captures the DNA mobility over a wide range of molecular weights, gel concentrations and electric fields. Although this formula is empirical in nature, the terms in the equation can sometimes be interpreted in the context of biased reptation theory ${ }^{79}$.

While we have discussed Ogston sieving and biased reptation separately, the transition between regimes is actually quite smooth. For example, in figure 2-8, the 5 different plots show the change in separation mechanism from Ogston sieving to reptation according to the concentration of the gel at an applied electric field of $270 \mathrm{~V} / \mathrm{cm}$. As can be seen in the plots (from left to right) the mobility decreases when the density of the polymer matrix is inceased, resulting in a higher resolution and a smaller range of DNA lengths which can be separated. 


\section{Introduction}

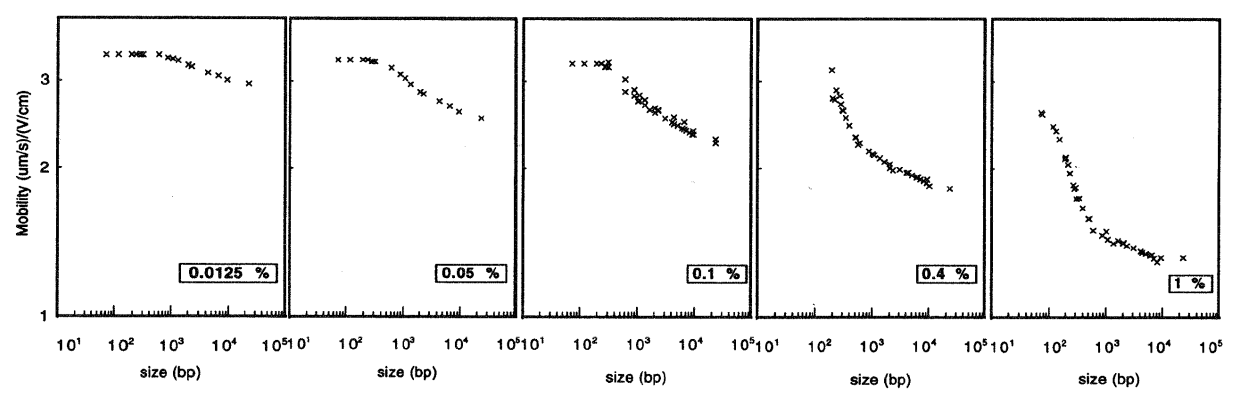

Figure 2-8 The transition from the Ogston sieving regime to the biased reptation regime. The mobility of various length DNA molecules at different percentages of the polymer hydroxyl propyl cellulose (HPC) is plotted. As can be seen from left to right, the mobility decreases when the percentage of the polymer is increased (and thus the density of the matrix is increased). Also, the resolution is increased by increasing the percentage of polymer. However as can be seen in last plot, large molecules ( $>\mathrm{kbp}$ ) have a very small difference in mobility, which results from the orientation of the molecule in the biased reptation regime. In the first plot, small DNA molecules cannot be separated at low matrix densities, but separation is possible at higher density. Reprinted with permission from ref. 119

\subsubsection{Entropic trapping $\left(R_{g} \sim b\right)$}

In this regime the pore dimensions are comparable to the dimensions of the radius of gyration of the molecule. As was the case in Ogston sieving, the inhomogeneity in a gel leads to some of the pore sizes being commensurate with the radius of gyration of the chain; most of the pores will be larger or smaller than the chain. Note that it is possible to make a uniform pore structure via colloidal templating ${ }^{120}$. Indeed, the latter study constitutes a definitive proof of entropic trapping, at least in the absence of an electric field. The existence of pore dimensions comparable to the radius of gyration provides two possibilities when the molecule is pulled through the gel by the applied electric field. First, the complete chain can be distributed among several pores (thus stretching the molecule), and second the molecule can be squeezed into one single pore. In a gel, usually, the pore size varies. This will provide a preference of the molecule for the larger pores, where the entropy loss is smallest. Squeezing the entire molecule in such a single pore will provide a minimal entropy loss, which is favored above stretching. In other words, larger pores can be seen as entropic traps. This phenomenon was first reported by Baumgartner ${ }^{121}$ and Muthukumar ${ }^{122}$ from computer simulations. Rousseau et al. ${ }^{123}$ showed the existence of an entropic trapping regime in polyacrylamide gel electrophoresis (PAA). When the applied electric field is low compared to the entropic trapping energy, the traps will decrease the mobility of the DNA molecules in a size-dependent fashion ${ }^{121,122,124,125}$. Entropic trapping is typical for highly concentrated gels, low fields and polymers which are flexible, as is the case for DNA. In the context of the ratio of radius of gyration to nominal pore size, the entropic trapping regime can be seen as a regime intermediate between the Ogston sieving regime and the biased reptation regime. 


\subsubsection{Nanostructure electrophoresis}

Having reviewed the extant theory for DNA electrophoresis in gels, we will now review recent results on DNA electrophoresis in nanostructures. We will classify the devices according to the regimes above (free-solution, Ogston sieving, reptation and entropic trapping) to make clear the connection (or lack thereof) between the gel electrophoresis theories and the phenomena observed in nanostructures.

The last few years have witnessed a number of experiments on DNA electrophoresis in nanoslits ${ }^{11-22}$, nanochannels ${ }^{23-34}$, channels with nanosize entropic traps and pillars ${ }^{35-45}$ and with nanopores and other structures ${ }^{46-50}$. The materials used for these nanofluidics experiments can develop a relatively strong surface potential depending on the buffer used for the experiment. For example, at $\mathrm{pH}>4$ the silanol groups of fused silica tend to dissociate, leaving a negatively charged surface. Counterions (in this case cations) are attracted to the negatively charged surface, leading to the formation of the Debye layer. While the Debye layer in a typical electrophoresis buffer is normally small relative to characteristic length scale of a microchannel, this is not always the case in a nanochannel. Upon decreasing the channel diameter, the number of surface charges per unit area begins to approach the number of mobile charges per unit volume of the bulk electrolyte. As a result the ratio of counter-ions to co-ions is changing because of the electroneutrality requirement. In figure $2-9$, the differences in the distribution of electric potential and ionic concentrations in both a microchannel and a nanochannel are presented. This figure illustrates that the electrical double layer can play an important role in nanostructures. Generally the electrical double layer is modeled by a Stern layer and a diffuse layer (figure 2-10). The Stern layer is the layer of immobilized counter ions very close to the charged surface of the channel. In the second layer or diffusive layer, the counter ions experience the (electrical) attraction of the surface, but less strongly when compared to the Stern layer, and as a result are able to diffuse freely. The border between the diffuse layer and the mobile layer is roughly equated in the shear plane, which is the plane where the liquid when pumped through the channel shears the wall.

The zeta potential, $\zeta$, is defined as the potential at the shear plane and is strongly dependent of the ionic strength of the solution. According to the Debye-Höckel theory, the potential decreases exponentially with distance $x$ from the plane of shear (equation 22 and figure 2$10)$.

$$
V_{x}=\zeta \cdot \exp \left(-x / \lambda_{D}\right)
$$

If the double layers are overlapping, as in the bottom picture of figure 2-9, this potential will not decay to zero in the entire nanochannel. In this way the counterions dominate and ion-selective nanochannels are created. This is not a new phenomenon - commercial ion selective membranes have been in production for a number years based on exactly the same physics. Rather, the novelty of studying electrokinetics in nanofabricated systems resides 
in the ability to (i) systematically vary the channel geometry and (ii) produce precise, simple geometries that are more amenable to theoretical modeling than many membrane technologies.

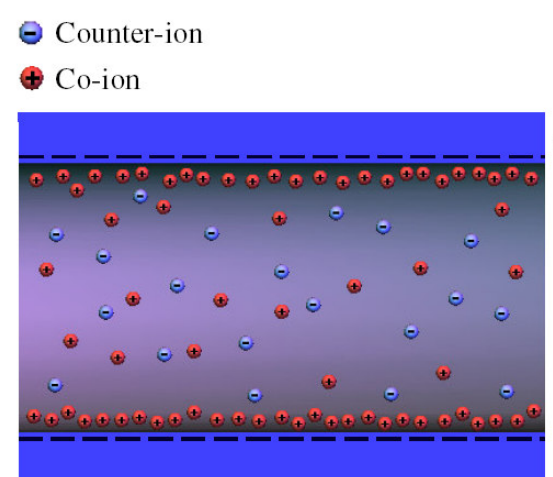

\section{Electrical}

potential $\Phi$

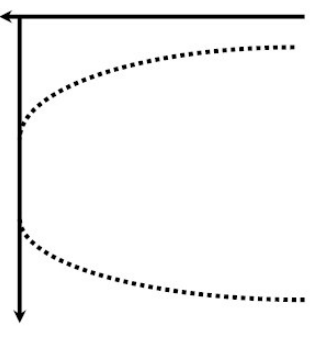

Microchannel
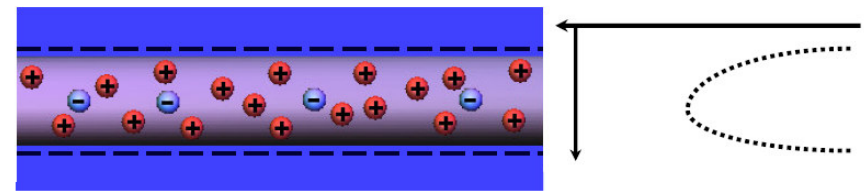

Ionic

\section{Concentration}
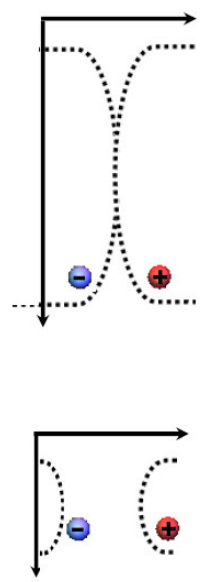

Nanochannel

Figure 2-9 The differences between a microchannel and a nanochannel. In a nanochannel the surface to volume ratio is much larger resulting in a comparable quantity of both surface charges and bulk charges. This leads to double layer overlap and a predominant presence of counterions in the channel. Picture after reference 126.

In our discussion thus far, we have only considered nanofluidic phenomena related to the electrical properties of the channel walls and solution ionic composition. We will now return to DNA, which is a large charged molecule. If such a molecule enters a nanostructure as for example a very tight nanoslit with a height of $20 \mathrm{~nm}$, the molecule has to also overcome an entropic barrier, since a large number of 3D conformations become forbidden. Once inside the channel, it will experience friction with the environment and the fluid inside this structure (just like within a network of pores inside a gel). Also, the DNA transport and conformations will be influenced by the charged surface and the resulting potential in the electrical double layers. In this section of the chapter, we will examine how the theories of gel electrophoresis can (or cannot) be applied to understand the nanostructure electrophoresis studies listed in the table at the end of this chapter. 


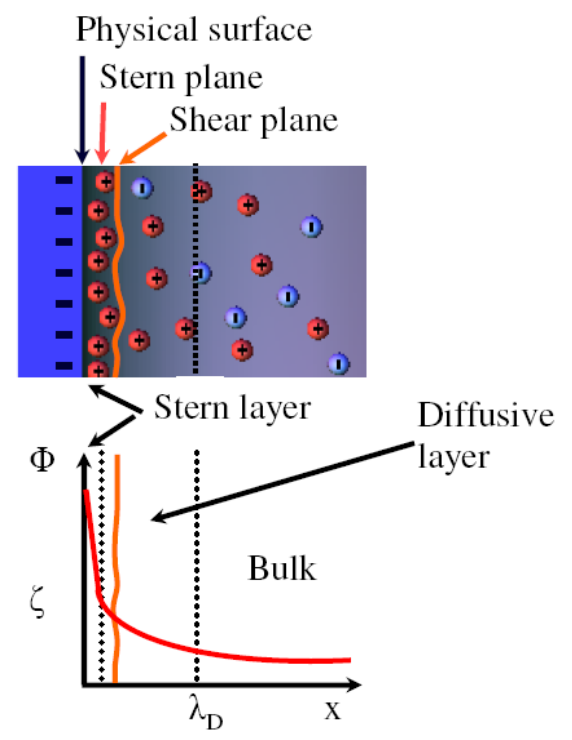

Figure 2-10 The electrical double layer (EDL). The EDL can be divided in a Stern layer and a diffuse layer. The thickness of the diffuse layer is characterized by the Debye length, which in this figure is presented as $\lambda_{\mathrm{D}}$. The potential at the shear plane (or surface of shear) which is located between the Stern layer and the diffuse layer is called the zeta potential $\zeta$.

\subsubsection{Free solution electrophoresis in nanostructures}

As we saw earlier, DNA in free-solution should only be separated by size if the Debye layer is large compared to the size of the molecule, which limits the separation to around $400 \mathrm{bp}$. However, the mobility difference is not particularly large so it is quite difficult to resolve the fragments. As demonstrated by Pennathur et al ${ }^{20}$, small DNA fragments can be rapidly separated in a nanochannel due to the double layer overlap. They studied DNA from $10 \mathrm{bp}-100 \mathrm{bp}$. The corresponding contour lengths, $3.4-34 \mathrm{~nm}$, are smaller than both the persistence length and the channel heights $(40 \mathrm{~nm}, 100 \mathrm{~nm}$ and $1560 \mathrm{~nm})$. The molecules were end labeled with Fluorescein or contained fluorescein-12-dUTP. The applied electric fields were $100-200 \mathrm{~V} / \mathrm{cm}$. They observed that the migration times depended on the ratio of the length of the DNA molecule, the half-depth of the channel and the ratio of the Debye length to the half depth. Best separation results for these fragment sizes were achieved in $100 \mathrm{~nm}$ fused silica nanochannels using sodium borate buffers at final concentrations of $1-10 \mathrm{mM}$. Pennathur et al. ${ }^{20}$ also compared gel free electrophoresis in a $100 \mathrm{~nm}$ high nanochannel to a $50 \mu \mathrm{m}$ high microchannel. Their results are reproduced in figure 2-11. Although we would expect to see some separation of the DNA in the $50 \mu \mathrm{m}$ microchannel, the length of the microchannel is in this case probably too small to perform separation of these small DNA molecules. 


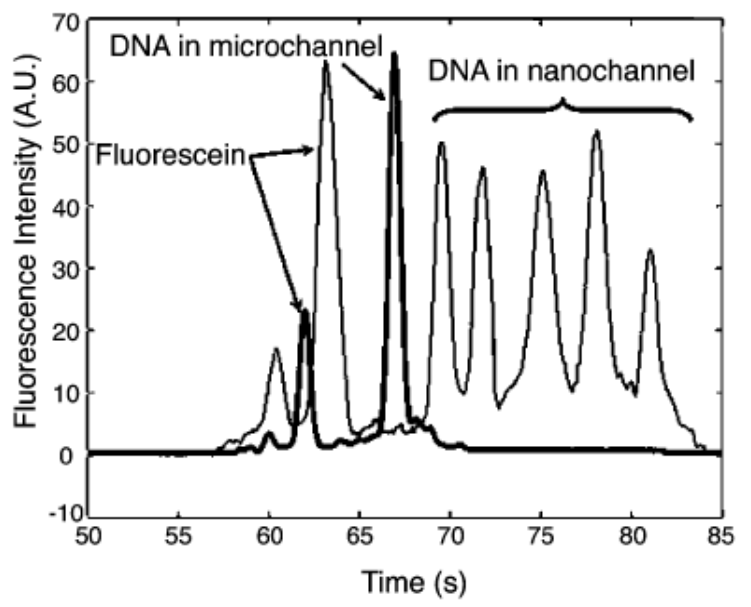

Figure 2-11 A comparison of a free solution separation provided in a $100 \mathrm{~nm}$ high nanochannel (thin line) and a $50 \mu \mathrm{m}$ high microchannel (thick line). The concentration of the sodium borate used was $10 \mathrm{mM}$ and the applied electric field $100 \mathrm{~V} / \mathrm{cm}$. It can be seen that the nanochannel provided a reasonable resolution over a separation distance of $20 \mathrm{~mm}$, while the results of the microchannel showed no separation at all over a distance of $23 \mathrm{~mm}$. The peaks of the DNA in the nanochannel are from fluorescein labeled nucleotide dUTP, $10 \mathrm{bp}, 25 \mathrm{bp}, 50 \mathrm{bp}$ and $100 \mathrm{bp}$ respectively. With permission reprinted from ref. ${ }^{20}$.

During the migration of DNA molecules through nanostructures, there is an interplay between the properties of the molecule and the electrical double layers. Also, steric and hydrodynamic effects influence the migration of the DNA molecule. Thus it is to be expected that the size range of the DNA molecules which can be separated with a certain nanostructure is related to the dimensions of the nanostructure. With the channels of 100 $\mathrm{nm}$ height, free solution separation of very small DNA fragments is possible.

Another interesting investigation was done by Campbell et al. ${ }^{50}$, however here only the influence of the confinement on $\lambda$ - DNA molecules was studied. They manufactured nano-capillaries with widths and depths down to $150 \mathrm{~nm}$ x $180 \mathrm{~nm}$. When comparing the mobilities of the $\lambda$ - DNA molecules, it was found that the capillaries with the smallest dimensions provided the highest mobilities. Even so, the velocity of the molecules could be controlled by the applied electric field. More examples of this relation between DNA size and structure size will be presented later.

Before we consider DNA electrophoresis in nanostructures, it is useful to conclude here with some of the advantages of the aforementioned methods. The important advantage of free flow electrophoresis is the speed of separation. The absence of a gel matrix results in another, perhaps even more important gain in time (no filling of capillaries). 


\subsubsection{Electrostatic sieving, Ogston Sieving and entropic trapping}

Even in the simple case of a straight nanochannel, one can still observe size and/or chargedependent sieving. The three important sieving mechanisms which can occur in nanostructures are presented in figure 2-12. Electrostatic sieving (A) occurs for small molecules (i.e., when the radius of gyration is smaller than the diameter or height of the capillary or channel) under conditions of double layer overlap. It is caused by electrostatic repulsion of the DNA molecules from the walls (ion exclusion). Since size-dependent sieving will always also occur, the second (B) regime cannot be separated from the first. The second (B) and the third regime $(\mathrm{C})$ are Ogston sieving and entropic trapping.

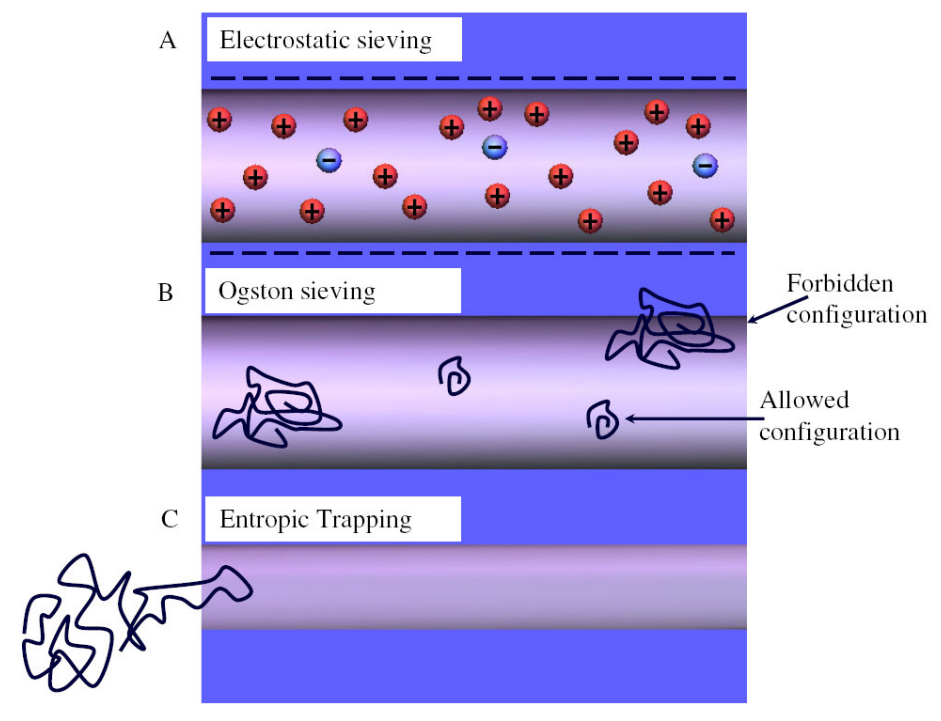

Figure 2-12 Three different regimes which can be distinguished in nanostructure DNA electrophoresis. The first regime is called electrostatic sieving (A). Here ions can be selected by using the surface charges and the electrical double layers. In B, Ogston sieving is presented. Smaller DNA molecules are able to pass through a nanostructure with a higher velocity than larger molecules. In $\mathrm{C}$, entropic trapping is presented. Entering the confined region requires overcoming an entropic barrier. Picture after reference 126

One of the simplest ways to implement these sieving mechanisms in a nanostructure is to make a periodic array of thin slits connected by deep wells. This geometry was originally used to separate long DNA via entropic trapping with a deep region several microns deep and a slit depth of $100 \mathrm{~nm}{ }^{37}$. More recently, downsizing both regions to the $10-100 \mathrm{~nm}$ regime has permitted the separation of shorter DNA and proteins in the so-called nanofilter 35. An example of a nanofilter (entropic trapping) array is presented in figure 2-13. The deep regions are called wells. The depth of the shallow and deep region is given by $\mathrm{d}_{\mathrm{s}}$ and 
$d_{w}$ respectively. The total period $\left(l_{t}\right)$ of one nanofilter is the length of the well $\left(1_{w}\right)$ and the shallow region $\left(l_{\mathrm{s}}\right)$ together. As a result of the geometry in the device, the electric field is not uniformly distributed. Inside the shallow regions, the electric field is the strongest, however the electric potential $\Phi$ monotonically decreases through the nanofilter. Depending on the length scale, at low electric fields the nanofilter can either operate in an Ogston sieving regime or in an entropic trapping regime. Entropic sorting was investigated by $\mathrm{Han}$ et al ${ }^{36,37,127}$ whereas $\mathrm{Fu}$ et al. ${ }^{35,40,38}$ and $\mathrm{Li}^{39}$ investigated Ogston sieving and entropic trapping. While the system illustrated in figure 2-13 operates in one dimension, it is also possible to pattern nanofilters in an asymmetric configuration to achieve continuous separations by the same sieving mechanisms ${ }^{38}$. An excellent review of these investigations is presented by Han et $\mathrm{al}^{127}$.

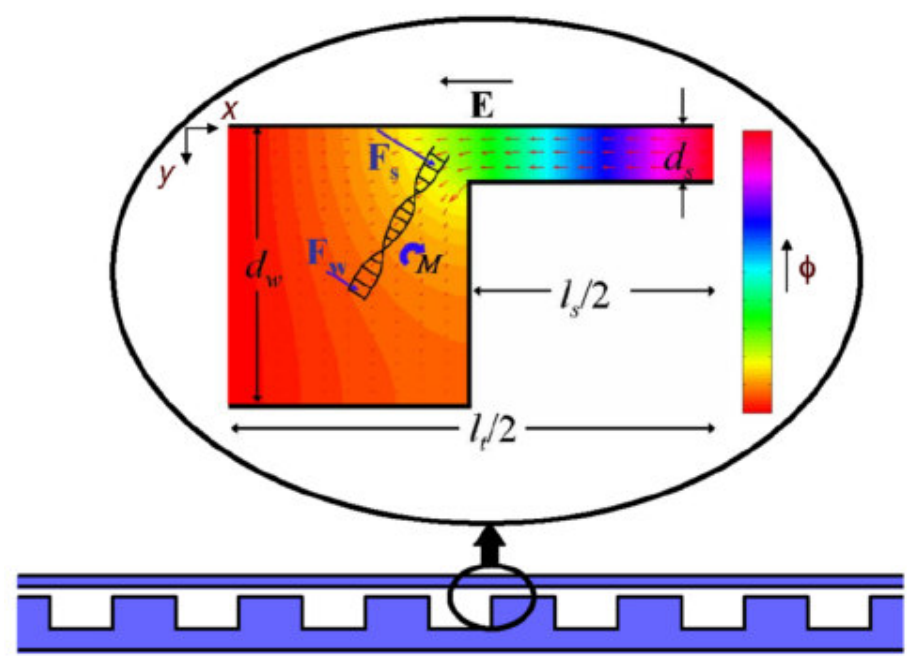

Figure 2-13 A nanofilter array, existing of an array of deep and shallow regions. The deep regions are called wells. It can be divided in unit cells of which one is presented in the inset. The total period $\left(l_{t}\right)$ of one nanofilter is the length of the deep region $\left(1_{w}\right)$ and the shallow region $\left(l_{s}\right)$ together. The depths of the shallow and deep region are given by $d_{s}$ and $d_{w}$ respectively. The electric potential is indicated by $\Phi$, where the color codes for the strength. As a result of the geometry of the device, the electric field is not uniform. Inside the shallow regions, the electric potential will be the highest. This non uniform electric field will exert forces $\left(\mathrm{F}_{\mathrm{s}}\right.$ and $\left.\mathrm{F}_{\mathrm{w}}\right)$ on the DNA molecule and resulting in a torque M. Reprinted with permission from ref. 133.

The first of such arrays were developed to separate large DNA molecules by entropic trapping. In these systems, the radius of gyration of these molecules was smaller than the well and much larger than the shallow regions. Remarkably, the larger molecules were able to migrate within a shorter time than the smaller molecules. In the simplest model of the separation process, the higher mobility for the large DNA was rationalized by the rate constant for forming a "beachhead" of DNA in the slit, which is kinetically favorable for larger molecules ${ }^{36}$. The physics of the entropic trapping system is a rich subject and has 
Chapter 2

been studied extensively by simulations ${ }^{128-132}$. Most importantly, these simulations allow one to account for the inhomogeneous field inside the well, which plays a key role in the escape process. When this sieving structure is further reduced to a nanofilter scale, with $325 \mathrm{~nm}$ deep wells and $73 \mathrm{~nm}$ slits ${ }^{40}$, it is possible to identify a cross-over between an Ogston sieving regime, where the small molecules have the higher mobility, and an entropic trapping regime, where the large molecules have the higher mobility. Figure 2-14 reproduces the mobility versus molecular weight data obtained by $\mathrm{Fu}$ et al. ${ }^{40}$. For this particular geometry, the crossover from Ogston sieving to entropic trapping occurs around $1.5 \mathrm{kbp}$. To relate this to the radii of gyration of the DNA, Fu et al. ${ }^{40}$ calculated the radius of gyration with the Kratky-Porod model, a general model that can be used to describe the conformation of semiflexible chains. This provided a radius of gyration of around $80 \mathrm{~nm}$ for a DNA length of $1.5 \mathrm{kbp}$. It can therefore be concluded from the results presented in figure 2-14 that the crossover occurs around $\mathrm{R}_{\mathrm{g}} / \mathrm{d}_{\mathrm{s}} \sim 1$. It can furthermore be seen that in the devices of these dimensions the mobility saturates at high fields. For example, in the particular geometry used by Fu et al. ${ }^{40}$, the mobility for molecules larger than $5 \mathrm{kbp}$ is independent of molecular weight.

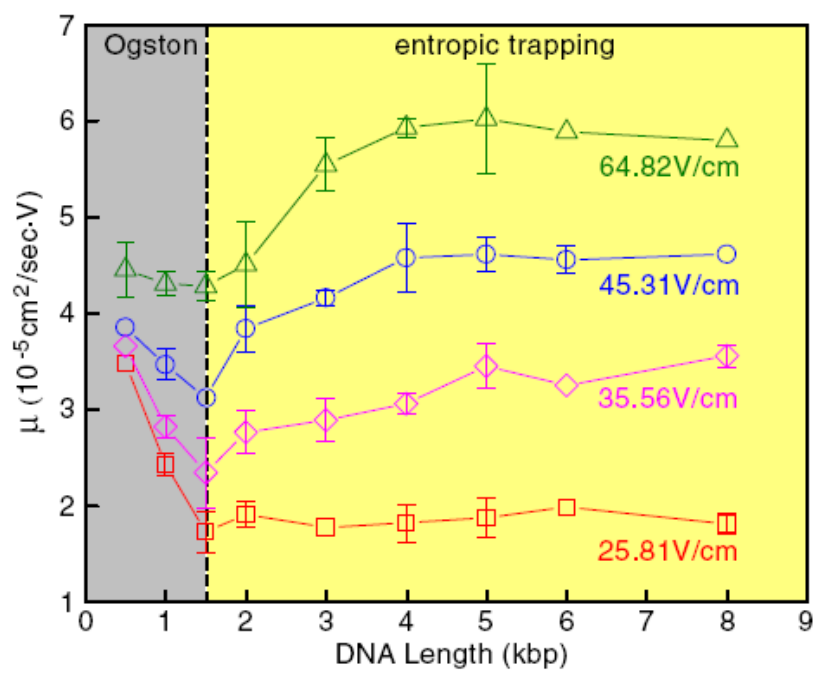

Figure 2-14 The crossover from Ogston sieving (grey part) to entropic trapping (yellow part). The vertical dashed line represents the crossover border, which is around $1.5 \mathrm{kbp}$ for this device. The dimension of the used nanofilter array are for the deep region (or well) $d_{d}=325 \mathrm{~nm}, d_{s}=73 \mathrm{~nm}$ with a periodicity of $1 \mu \mathrm{m}$. Reprinted with permission from ref 40 .

The electric field also appears to play a different role in the Ogston sieving and entropic trapping regimes. In the Ogston sieving regime, the mobility difference between the small DNA and the slower, large DNA decays as the electric field increases, eventually leading to a loss of resolution ${ }^{35}$. The mobility plateau can be attributed to a breakdown in the Ogston sieving mechanism at higher fields, where the entropic penalty for entering the slit becomes negligible compared to the decrease in enthalpic energy due to motion in the 
electric field ${ }^{40}$. The situation is opposite in the entropic trapping configuration. At low applied fields, for example, no separation is obtained. For a fixed molecular weight, the mobility increases with electric field due to a lowering of the barrier towards entering the slit.

It is also illuminating to consider what happens to short (rod-like) DNA molecules as the field increases. While experiments $\mathrm{s}^{35}$ have demonstrated that the mobility of DNA in the $100 \mathrm{bp}$ range begins to saturate at fields around $100 \mathrm{~V} / \mathrm{cm}$, Laachi et al ${ }^{133}$ proposed that this is actually the onset of band-inversion. As indicated in figure 2-14, at high electric fields, the DNA will become trapped at the entrance to the slit if rotational diffusion causes the molecule to become misaligned with the slit entrance. However, due to the difference in the electric field between the well and the slit, the electric field leads to a torque on the molecule. Thus, in addition to electric field induced migration, the field also rotates the DNA to an acceptable conformation near the shallow region entrance. This behavior was confirmed by simulations, which predict that the relative mobility increases with the electric field for a fixed ratio of $\mathrm{L} / \mathrm{d}_{\mathrm{s}}$. The model also predicts that longer DNA molecules (however still rigid), will exit the nanofilter array first when a high electric field is applied. While experiments on DNA electrophoresis in nanofilters at high fields indeed show this band-inverted behavior ${ }^{134}$, our understanding of the high-field electrophoresis in a nanofilter remains incomplete.

Based on the results presented in this section, it is clear that nanofilters could be a replacement for gel electrophoresis of short double-stranded DNA. Similar to gels, the key parameters controlling DNA electrophoresis in nanofilters are the dimensions of the array, the applied electric field and the length of the molecules to be separated. However, nanofilters possess a number of advantages over gels. In addition to their controllable and ordered geometries, nanofilters can be reused, and separations can be performed in less time.

\subsubsection{Biased reptation}

As discussed in the section on gel electrophoresis, biased reptation of DNA occurs when the molecules are inside a network of pores of which the pore dimensions are smaller than the radius of gyration of the molecule and an electric field is applied. The first investigations of DNA in nanostructures were performed by Volkmuth and Austin in 1992 ${ }^{135}$ using $150 \mathrm{~nm}$ deep nanochannels with micron-scale cylindrical obstacles. The purpose of these experiments was to mimic an agarose gel. They found that the mobility of the 5 $30 \mu \mathrm{m}$ long DNA molecules was independent of the DNA contour length $\mathrm{L}$ if no collisions with the obstacles occurred. However, as soon as the DNA molecules collide with the obstacles, their mobility became length dependent. This fundamental result has been confirmed by a number of subsequent separation experiments using micron-scale obstacles ${ }_{136,137}$ and submicron-scale obstacles ${ }^{44,45,138-141}$. In the context of nanopillar arrays, one particularly interesting question is the role of the array geometry ${ }^{44,45}$. In this investigation, 


\section{Chapter 2}

nanopillar arrays of which the distribution of the pillars was tilted or squared (see figure 2$15)$ were manufactured. DNA molecules (10 and $38 \mathrm{kbp}$; ApaI digested $\lambda$ - DNA) were seen reptating through this tilted distributed nanopillar structures, under the influence of an applied electric field of $50 \mathrm{~V} / \mathrm{cm}$. The movements of the molecules are described as a cycle of shrinking, stretching and hooking, which leads to a separation. When the DNA molecules moved in the squared distributed pillar array, the conformation of the molecules was not as predicted by reptation theory. Interestingly, the DNA molecules could not be separated in the squared distributed pillar array. Ogawa et al ${ }^{45}$ concluded that the reptilelike movement of DNA is very important for the separation of DNA by length. This suggests that the molecules are being delayed by the collisions according to their length while undergoing biased reptation with orientation through the tilted pillar structure. In contrast to this, the squared distributed pillar structure causes less collisions and thus the DNA molecules are only slightly deformed and the structures are to large to perform separation. Here again the possibility to manufacture entirely regular devices, with tilted or squared arrays, enables a better study of the fundamentals of biased reptation in a controlled geometry. The published results of Ogawa et al. ${ }^{44,45}$ of the separations were limited and therefore the capabilities of the manufactured devices should be further investigated.
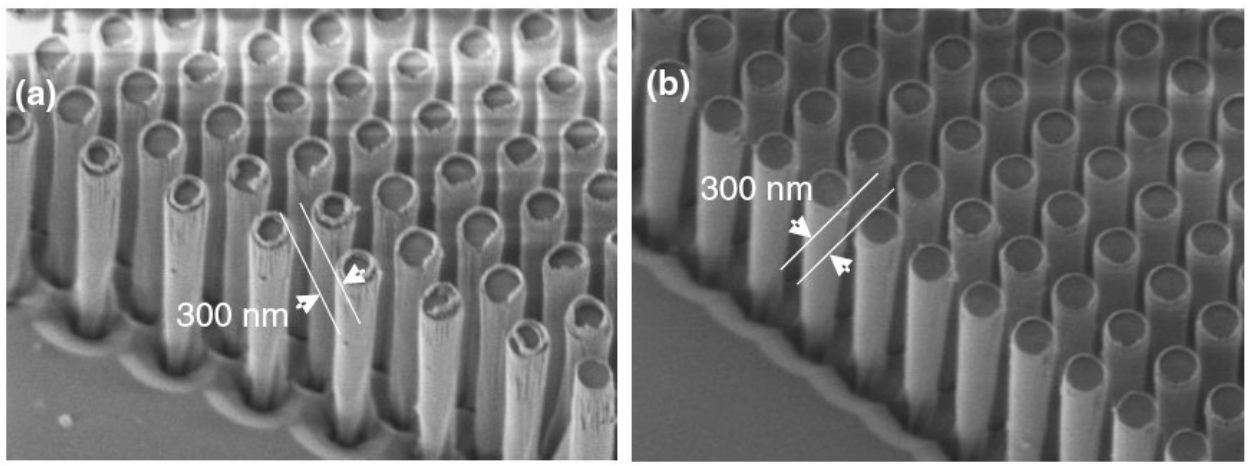

Figure 2-15 In A the distribution of the nanopillars is tilted, whereas the distribution in B is squared. Separation of DNA molecules was only possible with the tilted distribution, in which the molecules were reptating under influence of an electric field. In B, the molecules were changing their conformation, however no separation was realized. With permission reprinted from Ogawa et al. ${ }^{44}$.

Micropillar structures used for separations can also be used to "pre-stretch" the DNA prior to entering a nanofluidic area ${ }^{138}$. An example of such an application is presented in figure 2-16. Here, an area with micropillars is placed in front of a nanochannel array and will locally increase the electric field. The DNA molecules will partially uncoil as a result of the pillars ${ }^{43}$. The pre-stretching decreases the entropy barriers which the molecules have to overcome before they can enter a nanoslit and will thus simplify the introduction of the DNA molecules into the nanochannels. Nanopillar structures can also be used to study the uncoiling and recoiling behavior of DNA, as was done by Turner et al. ${ }^{42}$. 

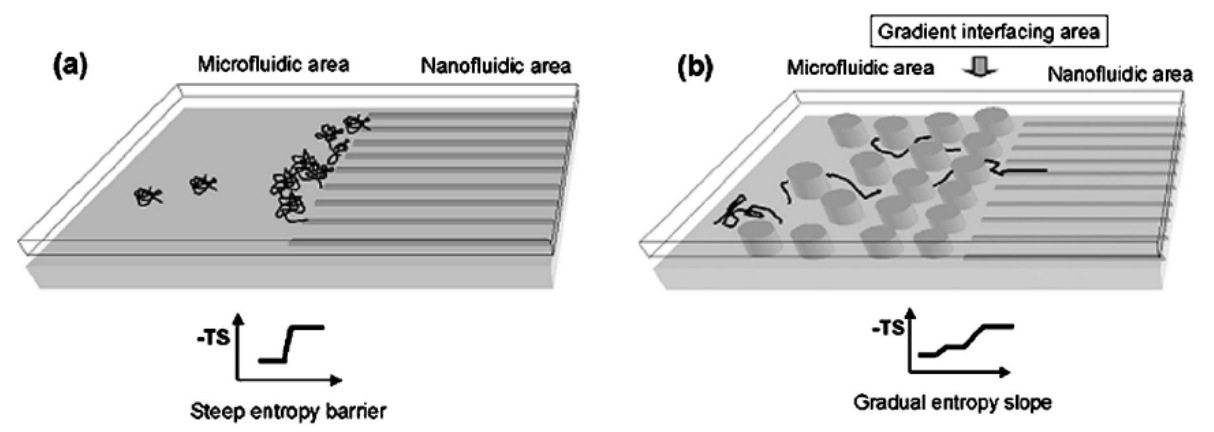

Figure 2-16 In A the coiled DNA molecules are forced into the nanochannel. This will result in a steep entropy barrier as can be seen in the graph below. In B, nanopillars in front of the entrance reduce the entropic barriers. The DNA molecules are pulled by the electric field, which results in uncoiling of the DNA molecules. As can be seen in the small graphs below the depicted devices, the entropy barrier to overcome is decreasing as a result of this uncoiling. With permission reprinted from ref. 43.

These nanopillars had a diameter of $35 \mathrm{~nm}$ and a center to center spacing of $160 \mathrm{~nm}$. The height of the channel was $60 \mathrm{~nm}$. DNA molecules were pulled into the nanopillar array by an electric field (see also figure 2-17).

(a)

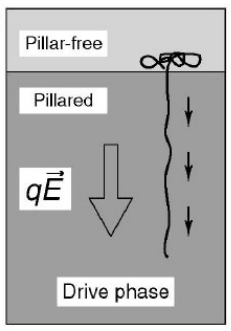

(b)

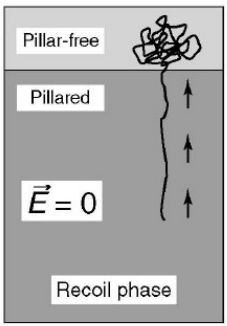

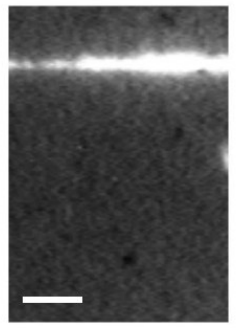

$t=0 s$

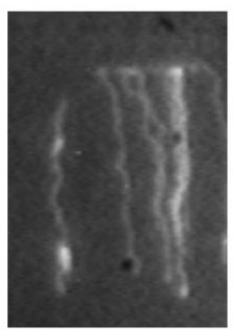

$\mathrm{t}=6.5 \mathrm{~s}$

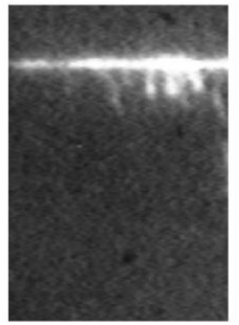

$t=1 \mathrm{~s}$

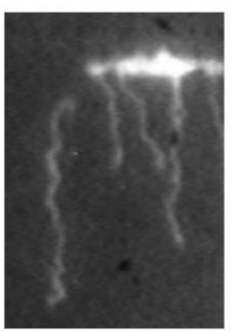

$\mathrm{t}=27.5 \mathrm{~s}$

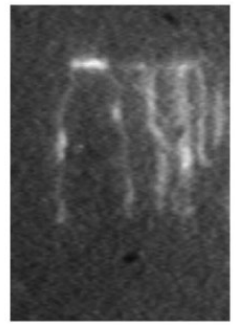

$\mathrm{t}=4 \mathrm{~s}$

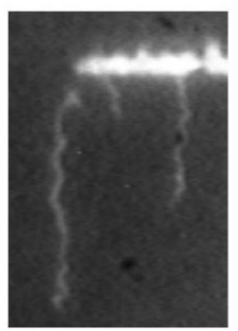

$\mathrm{t}=42.5 \mathrm{~s}$

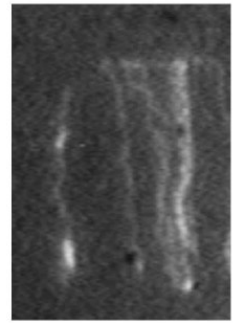

$t=6.5 s$

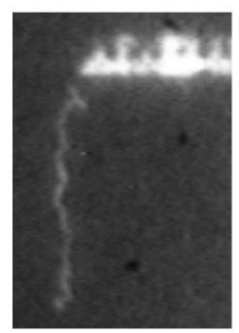

$\mathrm{t}=76.5 \mathrm{~s}$

Figure 2-17 DNA molecules are pulled into the pillared area by an applied electric field (A). When the electric field is turned of (B), the molecules which were partly inside the pillared area recoiled. Molecules which were entirely in the pillared area, remained there and showed only a slight contraction when the electric field was turned of. This slight contraction shows that the elastic entropy is negligible. Turner at al. ${ }^{42}$ suggested that in this way separation of large DNA molecules should be possible. Reprinted with permission from ref. 42 . 
Chapter 2

After the electric field was turned off, the DNA molecules which only partially entered the nanopillar array recoiled due to the lower entropic energy in the pillar free region. Molecules which where already completely in the nanopillar array, generally the shorter DNA, remained there. Turner et al. ${ }^{42}$ observed that molecules inside the pillar array, which remain there after turning the applied electric field off, do not experience a center of mass motion, only a slight contraction. According to Turner et al. ${ }^{42}$, this effect showed that the contribution of the elastic entropy is negligible and that a separation of large DNA molecules on length is possible, a result which was confirmed ${ }^{142}$.

\subsubsection{Electrophoresis in nanoslits and nanotubes}

Several authors have reported on DNA electrophoresis in the highly confined environment of nanoslits and nanotubes. Salieb-Beugelaar et al. ${ }^{18}$ presented an investigation of $\lambda$-DNA molecules in $20 \mathrm{~nm}$ high nanoslits. At low applied electric fields (below $300 \mathrm{~V} / \mathrm{cm}$ ) a more or less constant molecular extension of a few $\mu \mathrm{m}$ in the field direction and a fluent movement was observed, while at higher fields (above $300 \mathrm{~V} / \mathrm{cm}$ ), the molecular lengths strongly fluctuated and the movements of the molecules were intermittent. At low fields, the DNA therefore probably assumes a pancake shape, while at higher fields, the conformation continuously changes. The mobility was seen to strongly decrease with the applied field, which could not be explained. Cross et al. ${ }^{13}$ described the conformation of DNA molecules inside 19 and $70 \mathrm{~nm}$ high nanoslits as a pancake and presents a simple friction model with the walls to explain the observed decreased mobility. Another friction model was presented by Mannion et al. ${ }^{25}$, who investigated T4 DNA molecules (169 kbp) inside nanochannels of $90 \mathrm{~nm}$ wide and $100 \mathrm{~nm}$ height. The observed electrophoretic mobility of the stretched DNA molecules could with only partial success be described by a friction model of a cylinder inside a cylinder. The conclusion is that we still do not posses a satisfactory theoretical model for describing the electrophoretic transport of DNA in such strong confinement and further investigation is necessary. The control on the nanostructure dimensions certainly offers a huge advance here above the situation in gels.

The experimental parameters for the investigations discussed in the nanostructure electrophoresis section of this chapter are summarized in the table below. In the first column, the type of investigation is presented. The second and the third column provide information about the dimensions of the device and the type of DNA respectively. In the fourth and last column, the numbers of the references are given.

\subsection{Conclusions and perspectives}

The enormous experience and theoretical understanding gained about DNA molecules in gel electrophoresis has revealed the complexity of this elastic charged biopolymer as 


\section{Introduction}

it moves through networks of pores under applied electric fields. As was presented in this chapter, three main regimes are important in the separation of DNA molecules during gel electrophoresis, namely Ogston sieving, entropic trapping and biased reptation. These regimes are also relevant to investigations of nanostructure electrophoresis of DNA molecules. Instead of a network of pores, which provide confinement of the molecules in gels, it is now the nanochannel or slit which provides the confinement. Thus, the type of confinement can be quite different than in a gel. Nanostructures can be machined to have precise, periodic geometries, whereas gels are inherently random and possess a wide range of length scales. Also, the electric field direction and size can be controlled to a much larger extend in deterministic nanostructures, which can lead to new separation concepts. Nanostructures thus have an advantage over gels both for the study of fundamental phenomena and for manufacturing of devices precisely targeted to separate certain DNA sizes. 
Chapter 2

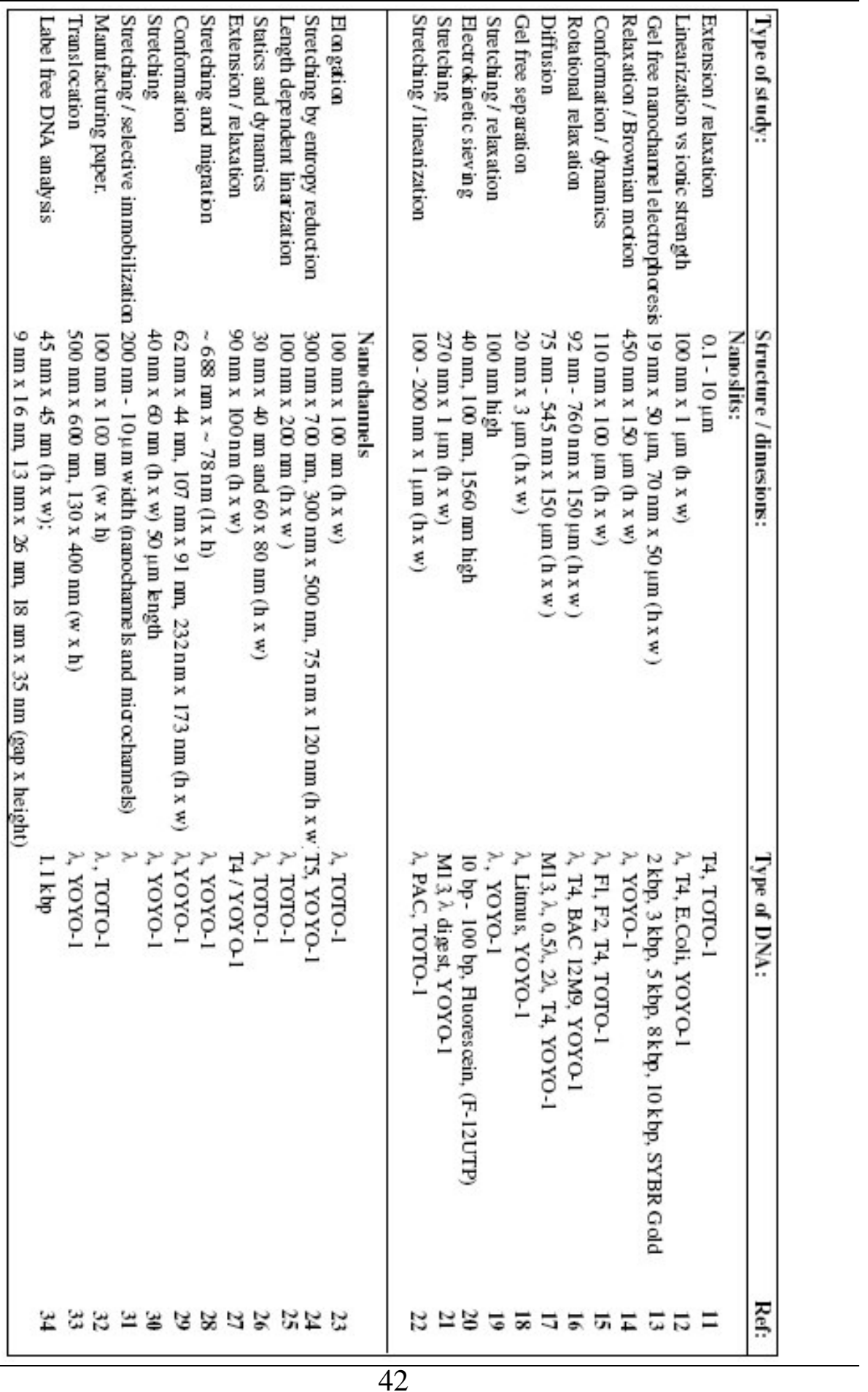




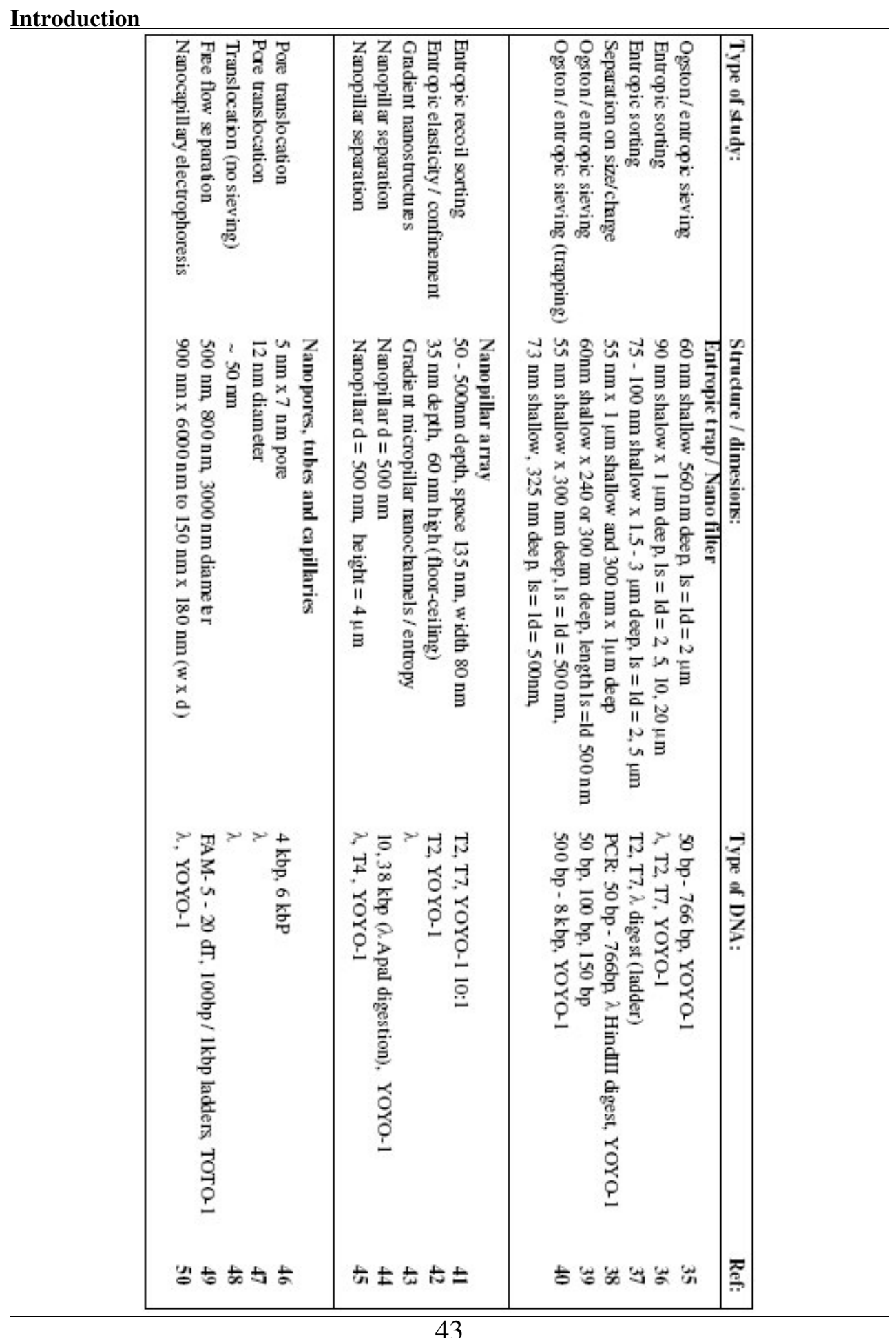




\subsection{References}

1. F. Persson, F. Westerlund, J.O. Tegenfeldt, A. Kristensen, Small 2009, 5, 2, 190 - 193.

2. R. Riehn, M. Lu, Y.M. Wang, S.F. Lim, E.C. Cox, R.H. Austin, Proc. Nat. Acad. Sci. USA, 2005, 102, 29, 10012 - 10016.

3. C.W. Kuo, K.H. Wei, C.H. Lin, J.Y. Shiu, P. Chen, Electrophoresis 2008, 29, 2931 2938.

4. L.R. Huang, E.C. Cox, R.H. Austin, J.C. Sturm, Science 2004, 304, 987 - 990.

5. S.W. Turner, A.M. Perez, A. Lopez, H.G. Craighead, J. Vac. Sci. Technol. B 1998, 16, $3835-3840$.

6. M.T. Blom, E. Chmela, R.E. Oosterbroek, R. Tijssen, A. van den Berg, Anal. Chem. 2003, 75, $6761-6768$.

7. J. P. Beech, J. O. Tegenfeldt, Lab on a Chip 2008, 8, 5, $657-659$.

8. Y.K. Jung, T.W. Kim, C. Jung, D.Y. Cho, H.G. Park, Small 2008, 4, 10, 1778 - 1784.

9. Y.K. Cho, J. Kim, Y. Lee, Y.A. Kim, K. Namkoong, H. Lim, K.W. Oh, S. Kim, J. Han, C. Park, Y.E. Pak, C.S. Ki, J.R. Choi, H.K. Myeong, C. Ko, Biosensors \& Bioelectronics 2006, 21, 2161 - 2169.

10. M.U. Kopp, A.J. de Mello, A. Manz, Science 1998, 5366, 1046 - 1048.

11. O. B. Bakajin, T. A. J. Duke, C. F. Chou, S. S. Chan, R. H. Austin, E. C. Cox, Phys. Rev. Lett., 1998, 80, 12, $2737-2740$.

12. K. Jo, D.M. Dhingra, T. Odijk, J. J. de Pablo, M. D. Graham, R. Runnheim, D. Forrest, D. C. Schwartz, Proc. Nat. Acad. Sci. USA, 2007, 104, 8, $2673-2678$.

13. J. D. Cross, E. A. Strychalski, H. G. Craighead, J. Appl. Phys., 2007, 102, 024701.

14. C. C. Hsieh, A. Balducci, P. S. Doyle, Nano Letters, 2008, 8, 1683 - 1688.

15. P. K. Lin, C. C. Fu, Y. L. Chen, Y. R. Chen, P. K. Wei, C. H. Kuan, W. S. Fann, Phys. Rev. E, 2007, 76, 011806.

16. C. C. Hsieh, A. Balducci, P. S. Doyle, Macromolecules, 2007, 40, 5196 - 5205.

17. A. Balducci, P. Mao, J. Han, P. S. Doyle, Macromolecules, 2006, 39, 6273 - 6281.

18. G. B. Salieb-Beugelaar, J. Teapal, J. van Nieuwkasteele, D. Wijnperlé, J. O. Tegenfeldt, F. Lisdat, A. van den Berg, J. C. T. Eijkel, Nano Lett., 2008, 8, 7, 17851790.

19. M. Krishnan, I. Mönch, P. Schwille, Nano Letters, 2007, 7, 1270 - 1275.

20. S. Pennathur, F. Baldessari, J. G. Santiago, M. G. Kattah, J. B. Steinman, P. J. Utz, Anal. Chem., 2007, 79, 8316-8322.

21. M. Foquet. J. Korlach, W. Zipfel, W. W. Webb, H. G. Graighead, Anal.Chem,2002, $74,6,1415-1422$.

22. R. Riehn, M. Lu, Y. Wang, S. F. Lim, E. C. Cox, R. H. Austin, Proc. Nat. Acad. Sci. USA, 2005, 102, 29, 10012 - 10016.

23. W. Li, J. O. Tegenfeldt, L. Chen, R. H. Austin, S. Y. Chou, P. A. Kohl, J. Krotine, J. C. Sturm, Nanotechnology, 2003, 14, $578-583$.

24. L. J. Guo, X. Cheng, C. F. Chuo, Nano Lett, 2004, 4, 1, 69 - 73.

25. J. O. Tegenfeldt, C. Prinz, H. Cao, S. Chou, W. W. Reisner, R. Riehn, Y. M. Wang, E. C. Cox, J. C. Sturm, P. Silberzan, R. H. Austin, Proc. Nat. Acad. Sci. USA, 2004, 101, $30,10979-10983$. 


\section{Introduction}

26. W. Reisner, K. J. Morton, R. Riehn, Y. M. Wang, Z. Yu, M. Rosen, J. C. Sturm, Phys. Rev. Let., 2005, 94, 196101.

27. J. T. Mannion, C. H. Reccius, J. D. Cross, H. G. Craighead, Biophys. J., 2006, 90, $4538-4545$.

28. D. Huh, K. L. Mills, X. Zhu, M. A. Burns, M. D. Thouless, S. Takayama, Nat. Mater, 2007, 6, $424-428$.

29. W. Reisner, J. P. Beech, N. B. Larsen, H. Flyvbjerg, A. Kristensen, J. O. Tegenfeldt, Phys. Rev. Let., 2007, 99, 058302.

30. K. Wang, S. Yue, L. Wang, A. Jin, C. Gu, P. Wang, H. Wang, X. Xu, Y. Wang, H. Niu, IEE Proc. Nanobiotechnol.,2006, 153, 1, 11 - 15.

31. B. Yang, V. R. Dukkipati, D. Li, B. L. Cardozo, S. W. Pang, J. Vac. Sci. Technolog. B., 2007, 25, 6, 2352- 2356.

32. W. Li, J. O. Tegenfeldt, L. Chen, R. H. Austin, S. Y. Chou, P. A. Kohl, J. Krotine, J. C. Sturm, Nanotechnology, 2003, 14, $578-583$.

33. D. Xia, T. C. Gamble, E. A. Mendoza, S. J. Koch, X. He, G. P. Lopez, S. R. J. Brueck, Nano Letters, 2008, 8, 6, 1610 - 1618.

34. X. Liang, S. Y. Chou, Nano Letters, 2008, 8, 5, $1472-1476$.

35. J. Fu, P. Mao, J. Han, Appl. Phys. Lett, 2005, 87, 263902.

36. J. Han, S. W. Turner, H. G. Craighead, Phys. Rev. Lett., 1999, 83, 8, 1688 - 1691.

37. J. Han, H. G. Craighead, Science, 2000, 28, 1026 - 1029.

38. J. Fu, R. B. Schoch, A. L. Stevens, S. R. Tannenbaum, J. Han, Nat. Nanotechno., 2007, 2, $121-128$.

39. Z. R. Li, G. R. Liu, Y. Z. Chen, J. S. Wang, H. Bow, Y. Cheng, J. Han Electrophoresis, 2008, 29, $329-339$.

40. J. Fu, J. Yoo, J. Han, Phys. Rev. Lett., 2006, 97, 018103.

41. S. M. Stavis, J. B. Edel, Y. Li, K. T. Samiee, D. Luo, H. G. Craighead, J. Appl. Phys., 2005, 98, 44903.

42. S. W. P. Turner, M. Cabodi, H. G. Craighead, Phys. Rev. Letters, 2002, 88, 12, 128103.

43. H. Cao, J. O. Tegenfeldt, R. H. Austin, S. Y. Chou, Appl. Phys. Lett., 2002, 81, 16, $3058-3060$.

44. R. Ogawa, N. Kaji, S. Hashioka, Y. Baba, Y. Horiike, Jap. Soc. Appl. Phys., 2007, 46, $2771-2774$.

45. R. Ogawa, H. Ogawa, A. Oki, S. Hashioka, Y. Horiike, Thin Solid Films, 2007, 515, $5167-5171$.

46. M. Gershow, J. A. Golovchenko, Nat. Nanotech., 2007, 775 - 779.

47. R. M. M. Smeets, U. F. Keyser, D. Krapf, M. Y. Wu, N. H. Dekker, C. Dekker, NanoLetters, 2006, 6, 1, 89 - 95.

48. R. Fan, R. Karnik, M. Yue, D. Li, A. Majumdar, P. Yang, Nano Letters, 2005, 5, 9, $1633-1637$.

49. X. Wang, S. Wang, V. Veerappan, C. K. Byun, H. Nguyen, B. Gendhar, R. D. Allen, S. Liu, Anal. Chem.,2008, 80, 14, 5583- 5589.

50. L.C. Campbell, M. J. Wilkinson, A. Manz, P. Camilleri, C. J. Humphreys, Lab on a Chip, 2004, 4, 225 - 229.

51. J.D. Watson, F.H.C. Crick, Nature 1953, 3, 171, 737 - 738. 
52. T. Odijk, J. Polym. Sci., 1977, 15, 477 - 483.

53. J. Skolnick, M. Fixman, Macromolecules, 1977, 10, 944 - 948.

54. G. S. Manning, Biophys. J. 2006, 91, $3607-3616$.

55. P.G. de Gennes, Scaling Concepts in Polymer Physics, Cornell University Press, Ithaca, NY, 1979.

56. D.W. Schaefer, J.F. Joanny, P. Pincus, Macromolecules 1980, 13, 1280 - 1289.

57. R.M. JendreJack, D.C. Schwartz, M.D. Graham, J.J. Pablo, J. Chem. Phys. 2003, 119, $1165-1173$.

58. D.E. Smith, T.T. Perkins, S. Chu, Macromolecules, 1996, 29, 1372 - 1373.

59. J. T. Mannion, H. G. Graighead, Biopolymers, 2006, 85, $131-142$.

60. N.C. Stellwagen, C. Gelfi, P.G. Righetti, Biopolymers, 1997, 42, 687 - 703.

61. P.J. Flory, S. Fisk, J. Phys. Chem., 1966, 44, 6, 2243 - 2248.

62. P.J. Flory, Science, 1975, 188, $1268-1275$.

63. G. S. Manning, J. Chem. Phys. 1969, 51, $924-939$.

64. G. S. Manning, Polyelectrolyte Binding 1979, 12, $443-449$.

65. G. S. Manning, Macromolecules 2007, 40, $8071-8081$.

66. J. L. Barrat, J.F. Joanny, Adv. Chem. Phys. 1996, 94, 1 - 66.

67. D.A. Hoagland, E. Arvanitidou, C. Welch, Macromolecules, 1999, 6180 - 6190.

68. W.B. Russel, D.A. Saville, W.K. Schowalter, ColoidalDispersions ,1989 (Cambridge University, Cambridge, England).

69. T. Odijk, Macromolecules 1983, 16, 1340 - 1344.

70. P. G. de Gennes, Macromolecules, 1976, 9, 587 - 593.

71. F. Brochard, P.G. de Gennes, J. Chem. Phys., 1977, 67, $52-56$.

72. M. Daoud, P.G. de Gennes, J. Phys. (Paris), 1977, 38, 85 - 93.

73. C.C.Hsieh, P.S. Doyle, Korea-Australia Rheology J., 2008, 127 - 142.

74. T. Odijk, Phys. Rev. E, 2008, 77, 06090.

75. C. Desruisseaux, D. Long, G. Drouin, G.W. Slater, Macromolecules, 2001, 34, 44 52.

76. G. S. Manning, J. Phys. Chem., 1981, 85, $1506-1515$.

77. C. Heller, C. Pakleza and J.L. Viovy, Electrophoresis, 1995, 16, 1423-1428.

78. J. L. Viovy, Rev. Mod. Phys., 2000, 72, 3, 813-872.

79. G. W. Slater, S. Guillouzic, M. G. Gauthier, J. F. Mercier, M. Kenward, L. C. McCormick, F. Tessier, Electrophoresis, 2002, 23, 3791 - 3816.

80. W. M. Deen, AIChE J., 1987, 33, 9, 1409 - 1425.

81. P.D. Grossman, D. S. Soane, Biopolymers, 1991 31, $1221-1228$.

82. T. Duke and J.-L. Viovy, Phys. Rev. E, 1994, 49, $2408-2416$.

83. C. Heller, Electrophoresis, 1998, 19, $1691-1698$.

84. L. Mitnik, L. Salome, J.-L. Viovy, C. Heller, J. Chromatogr. A, 1995, 710, 309 - 321.

85. C. Wu, M.A. Quesada, D.K. Schneider, R. Farinato, F.W. Studier, B. Chu, Electrophoresis, 1996, 17, 1103 - 1109.

86. C. Heller, Electrophoresis, 1999, 20, 1962 - 1977.

87. A.G. Ogston, Trans. Faraday Soc., 1958, 54, $1754-1757$.

88. J. C. Giddings, E. Kucera, C.P. Russel, M.N. Myers, J. Phys. Chem., 1968, 72, 4397 4408.

89. C.J.O.R. Morris, Prot. Biol. Fluids 1966, 14, 543 - 551. 


\section{Introduction}

90. D. Rodbard, A. Chrambach, Proc. Natl. Acad. Sci. 1970, 4, 970 - 977.

91. M.G. Gauthier, G.W. Slater, Electrophoresis, 2003, 24, 3, 441 - 451.

92. M.G. Gauthier, G.W. Slater, J. Chem. Phys. 2002, 117, 14, $6745-6756$.

93. J.F. Mercier, F. Tessier, G.W. Slater, Electrophoresis, 2001, 22, 13, 2631 - 2638.

94. J.F. Mercier, G.W. Slater, Macromolecules, 2001, 34, 10, 3437 - 3445.

95. J. Boileau, G.W. Slater, Electrophoresis, 2001, 22, 4, 673 - 683.

96. J. Labrie, J.F. Mercier, G.W. Slater, Electrophoresis, 200, 21, 5, 823 - 833.

97. J.F. Mercier, G.W. Slater, Electrophoresis, 1998, 19, 10, 1560 - 1565.

98. G.W. Slater, J.R. Treurniet, J. Chrom. A., 1997, 772, 39 - 48.

99. G.W. Slater, H.L. Guo, Electrophoresis, 1996, 17, 9, 1407 - 1415.

100.G.W. Slater, H.L. Guo, Electrophoresis, 1996, 17, 6, 977 - 988.

101.P. J. de Gennes, J. Chem. Phys., 1971, 55, 572 - 579.

102.L.S. Lerman, H. L. Frisch, Biopolymers, 1982, 21, 995 - 997.

103.O.J. Lumpkin, B. H. Zimm, Biopolymers, 1982, 21, 2315 - 2316.

104.A.N. Semenov, J. F. Joanny, Phys. Rev. E, 1997, 55, 789 - 799.

105.G.W. Slater, J. Noolandi, Biopolymers, 1986, 25, 431 - 454.

106. Noolandi, J., J. Rousseau, G. W. Slater, C. Turmel, and M. Lalande, Phys. Rev. Lett., $1987,58,2428-2431$.

107.T.A.J. Duke, J. Chem. Phys., 1990, 93, 9049 - 9055.

108.T.A.J. Duke, J.-L. Viovy, Phys. Rev. Lett., 1992, 68, 542 - 545.

109.G.T. Barkema, J. F. Marko,B. Widom, Phys. Rev. E , 1994, 49, 6, 5303 - 5309.

110.T.A.J. Duke, A.N. Semenov, J.L. Viovy, Phys. Rev. Lett., 1992, 69, 3260 - 3263.

111.A.N. Semenov, T. A. J. Duke, J. L. Viovy, 1995, Phys. Rev. E, 1995, 51, 2, 15201537.

112.T.A.J. Duke, J.L. Viovy, A.N. Semenov, Biopolymers, 1994, 34, 239 - 247.

113.C. Heller, T.A.J. Duke, J.L. Viovy, Biopolymers, 1994, 34, 249 - 259.

114.A. Pluen, B. Tinland, J. Sturm, G. Weill, Electrophoresis, 1998, 19, 1548 - 1559.

115.J. Yan, N. Best, J.Z. Zhang, H. Ren, R. Jiang, J. Hou, N.J. Dovichi, Electrophoresis, 1996, 17, 1037 - 1045.

116.J.L. Viovy, Mol. Biotechnol., 1996, 6, 31- 46.

117.N. Lee, S. Obukhov, M. Rubinstein, Electrophoresis, 1996, 17, 1011 - 1017.

118.D.H. van winkle, A. Beheshti, R.L. Rill, Electrophoresis, 2002, 23, 15 - 19.

119.A.E. Barron, C. Heller, 1997, Vieweg Press, Amsterdam, The Netherlands.

120.D. Nykypanchuk, H. H. Strey and D. A. Hoagland, Science, 2002, 297, 987 - 990.

121.A. Baumgartner, M. Muthukumar, J. Chem. Phys., 1987, 87, 3082 - 3088.

122.M. Muthukumar, A. Baumgartner, Macromolecules, 1989, 22, 1937 - 1941.

123.J. Rousseau, G. Drouin, G.W. Slater, Phys. Rev. Lett., 1997, 79, 10, 1945 - 1948.

124.G.W. Slater, H.L. Guo, Electrophoresis, 1995, 16, 11 - 15.

125.G.I. Nixon, G.W. Slater, Phys. Rev. E,1996, 53, 4969 - 4980.

126.P. Abgrall, N. T. Nguyen, Anal. Chem., 2008, 80, 2326 - 2341.

127.J. Han, J. Fu, R.B. Schoch, Lab on a Chip 2008, 8, 23 - 33.

128.F. Tessier, J. Labrie, G.W. Slater, Macromolecules, 2002, 35, $4791-4800$.

129.Z. Chen, F.A. Escobedo, J. Mol. Sim., 2003, 29, 417 - 425.

130.M. Streek, F. Schmid, T.T. Duong, A. Ros, J. Biotechnol., 2004, 112, 79 - 89.

131.S.H. Kim, A. S. Panwar, S. Kumar, K.H. Ahn, S. J. Lee, J. Chem. Phys., 2004, 121, 


\section{Chapter 2}

$9116-9122$.

132.A.S. Panwar, S. Kumar, Macromolecules, 2006, 39, 1279 - 1289.

133.N. Laachi, C, Declet, C. Matson, K. D. Dorfman, Phys. Rev. Lett., 2007, 98, 098106.

134.E.A. Strychalski, H.W. Lau, L. Archer, H.G. Craighead, Proceedings of the 12th International Conference on Miniaturized Systems for Chemistry and Life Sciences (uTAS), San Diego, CA USA, October 12-16, 2008, 856 - 858.

135.W.D. Volkmuth and R.H. Austin, Nature, 1992, 358, $600-602$.

136.P. S. Doyle, J. Bibette, A. Bancaud, J.-L. Viovy, Science, 2002, 295, 2237.

137.N. Minc, C. Fuetterer, K. D. Dorfman, A. Bancaud, C. Goubault, C. Gosse, J. L Viovy, Anal. Chem., 2004, 76, 3770 - 3776.

138. N. Kaji, Y. Tezuka, Y. Takamura, M. Ueda, T. Nishimoto, H. Nakanishi, Y. Horiike, Y. Baba, Anal. Chem., 2004, 76, 15 - 22.

139.Y. C. Chan, M. Carles, N. J Sucher, M. Wong, Y. Zohar, J. Micromech. Microeng., 2003, 13, $914-921$.

140.Y. C. Chan, Y.-K. Lee, Y. Zohar, J. Micromech. Microeng., 2006, 16, 699 - 707.

141.J. Shi, A. P. Fang, L. Malaquin, A. Pepin, D. Decanini, J.-L. Viovy, Y. Chen, Appl. Phys. Lett., 2007, 91, 153114.

142.M. Cabodi, S.W.P. Turner and H.G. Craighead, Anal. Chem., 2002, 74, 5169-5174. 
Filename: 2_Introduction_For_WPS_Final_Reserve

Directory: $\quad$ C:Locuments and Settings/Georgette.UTWENTE-

A174A94\Desktop\Ready for printing $\backslash$ Reserve

Template:

C:IDocuments and Settings $\backslash$ Georgette.UTWENTE-

A174A94\Application DatalMicrosoft|Templates $\backslash$ Normal.dot

Title: 1

Subject:

Author:

Georgette

Keywords:

Comments:

Creation Date: $\quad$ 9/29/2009 1:55:00 PM

Change Number: 2

Last Saved On: $\quad$ 9/29/2009 1:55:00 PM

Last Saved By: Georgette

Total Editing Time: 1 Minute

Last Printed On: $\quad$ 9/29/2009 1:55:00 PM

As of Last Complete Printing

Number of Pages: 34

Number of Words: $\quad$ 12,316 (approx.)

Number of Characters: $\quad 58,137$ (approx.) 


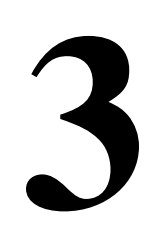

\section{Field Dependent DNA Mobility}

In this chapter investigations on the electrokinetic transport behaviour of $\lambda$-DNA in $20 \mathrm{~nm}$ high fused silica nanoslits is presented. DC electric fields are applied varying from 4 to 200 $\mathrm{kV} / \mathrm{m}$. We find that the mobilities are both field- and length-dependent. Furthermore, a change in transport behavior is found between low and high applied fields. In high fields the DNA molecules move in an intermittent way, whereas in low fields the molecules move in a fluent way. Different mobilities are found for $\lambda$-DNA and Litmus DNA, suggesting that electrophoresis in narrow nanoslits can be used for DNA separation.

Parts of this chapter was published in: G. B. Salieb-Beugelaar, J. Teapal, J. van Nieuwkasteele, D. Wijnperlé, J. O. Tegenfeldt, F. Lisdat, A. van den Berg, J. C. T. Eijkel, Nano Letters, 2008, 8, 7, 1785-1790. 


\subsection{Introduction}

The investigation of the behavior of confined DNA in a nanofluidic environment offers a promising way to explore its physical and structural properties ${ }^{1-10}$. The results can form the basis for new devices for DNA analysis, to improve diagnostics or to develop tools for the study of DNA-protein interactions ${ }^{11-12}$. General reviews useful in this context on DNA separation ${ }^{13-14}$, and on nanofluidics and nanotechnology ${ }^{15-17}$ have been published. DNA in a microfluidic environment will have a characteristic 3D "blob" shape. In a 2D nanofluidic environment ("nanoslit") DNA will not be able to take this shape but instead, must form a 2D pancake shape ${ }^{18-19}$. In a $1 D$ nanofluidic environment finally, DNA must stretch to a 1D "wormlike" molecule ${ }^{20}$. These nanofluidic conformations of DNA have been widely investigated in many different ways, and interpreted using models from polymer physics. The transport of these molecules furthermore has been explained with classical viscous friction models. Examples of phenomena observed are the compression and extension of single DNA molecules in nanochannels ${ }^{21}$, and the conformation change when the biopolymer is undergoing an entropically induced motion ${ }^{5}$. Still unexplained behavior of this molecule was recently described ${ }^{22}$ when silicon dioxide - glass nanoslits were used and a spontaneous stretching and attraction of DNA to the sidewall of the slits was found. Apart from conformational studies, the mobility of DNA in a nanofluidic environment has also been investigated several times. Recent papers report size-dependent mobility and possibly the start of the development of new gel free, size dependent separation devices ${ }^{23-}$ ${ }^{24}$. In the study presented here, we investigate the field-dependent mobility of DNA in 20 nm high nanoslits. We show that application of a DC electric field can strongly influence DNA mobility. As a possible explanation of the observed behavior we suggest dielectrophoretic and/or steric trapping.

\subsection{Experimental protocol}

\subsubsection{The nanoslit device}

Fused silica nanoslit devices with heights of $20 \mathrm{~nm}$ were manufactured as described in Appendix 1 at the end of this thesis. Of these devices both the surface roughness as the height of the nanoslits was studied.

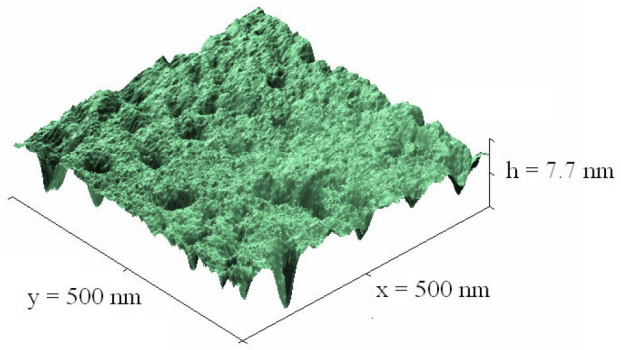

Figure 3-1. The surface roughness of the nanoslits as measured by AFM and using tips with a radius of $2 \mathrm{~nm}$. Although the rms value is between $0.8 \mathrm{~nm}-1.1 \mathrm{~nm}$, holes occur with a depth of down to 8 nm. 


\section{Field Dependent DNA Mobility}

\subsubsection{Electric setup}

The experimental setup was used as described in Appendix 1 at the end of this thesis. The Pt electrodes in the reservoirs were connected to a DC power supply (Keithley; high voltage source meter, model 2410). The YOYO-1 labelled $\lambda$-DNA/ Litmus molecules were pulled into the nanoslits by applying electric fields in DC between 6 and $200 \mathrm{kV} / \mathrm{m}$.

\subsection{Results and discussion}

DNA transport experiments were carried out at electric fields of $6 \mathrm{kV} / \mathrm{m}$ to $200 \mathrm{kV} / \mathrm{m}$. At low electric fields (below $30 \mathrm{kV} / \mathrm{m}$ ) a fluent movement of the $\lambda$-DNA molecules was observed in the nanoslits.

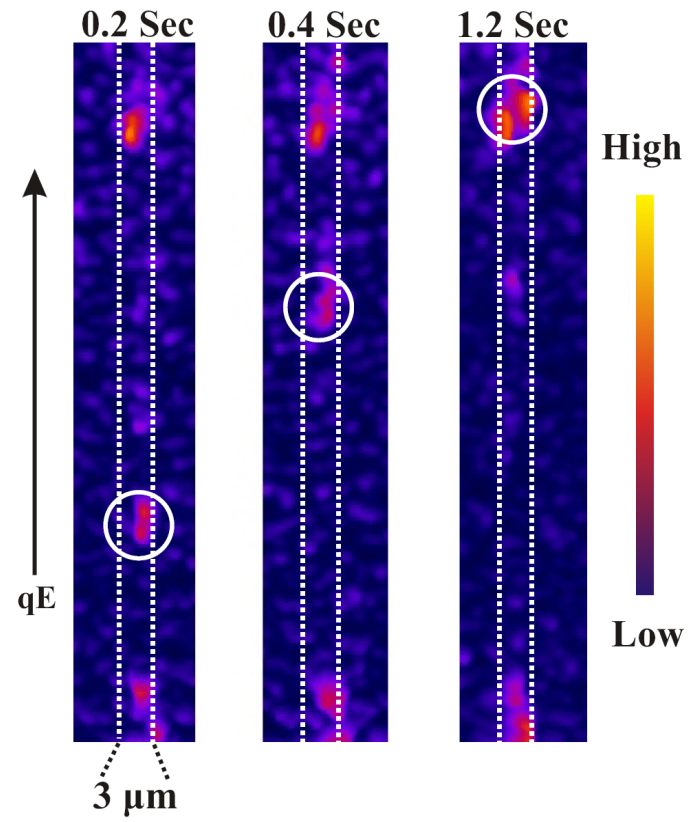

Figure 3-1 An example of a typical analysis of DNA movement is presented here in three different video frames. The number of frames during which an intermittently moving molecule would move vary between molecules. The tracked DNA is in the white circle. The color coding runs from yellow (high fluorescent emission) to blue (low fluorescent emission). Out of these data, plots as presented in figure 4 were created to calculate DNA mobility.

At high electric fields ( $30 \mathrm{kV} / \mathrm{m}$ and above) an intermittent movement of $\lambda$-DNA molecules was observed. Figure 3-1 shows three successive video frames of $\lambda$-DNA molecules in a nanoslit at an applied field of $200 \mathrm{kV} / \mathrm{m}$. One $\lambda$-DNA molecule (red area within a white circle) is moving in the three frames. The other, not moving yellow-red areas 
Chapter 3

represent $\lambda$-DNA which was permanently trapped during this experiment. From the displacement of the molecules (encircled by the white circles to visualize the DNA molecule tracked is presented in figure 3-1), the mobility of the center of mass of the DNA was calculated. Figure 3-2 shows an example of an intermittent movement as a distance / time graph. Part A in this graph represents the movement during the three frames displayed in figure 3-1. Part B represents a time interval within part A, in which the molecule was trapped. To study in detail the intermittent movement and the possible occurrence of traps and preferential pathways, the video frames were superimposed. An example of thus constructed pathways, from the video frames of the same experiment used in figure 3-1, is shown in figure 3-3. Roughly two different traps were found. First, traps existed where most $\lambda$-DNA molecules were delayed or temporarily trapped and second, traps where some of the molecules got trapped, whereas other molecules moved through fluently. Possible explanations for the trapping mechanisms could be steric trapping or dielectrophoresis ${ }^{25-26}$. Steric trapping can occur at places were the surface roughness is so large that it presents a mechanical obstacle in the slit. When DNA that is moving through the nanoslit as a result of the applied electric field meets the obstacle and is pulled into, around or against it by the electric field, it can be trapped in this way ${ }^{27}$. It subsequently will depend on the shape of the obstacle and the strength of the electric field, whether the $\lambda$-DNA is able to escape which will only be possible due to the thermal movement (diffusion). This type of trapping is expected to be highly field-dependent ${ }^{28}$, as is indeed observed.

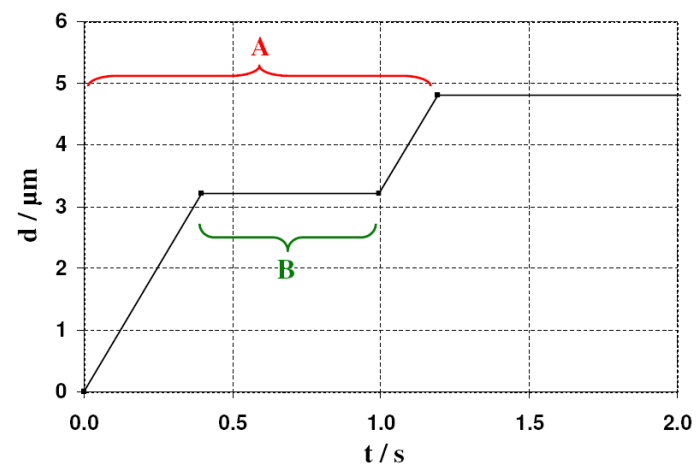

Figure 3-2. The intermittent movement of a single DNA molecule at a high electric field of 200 $\mathrm{kV} / \mathrm{m}$. The data provided out of the video frames of figure 3-1 were used to calculate part A of this graph. Part B represent the time in which the molecule was trapped.

Alternatively, temporary trapping may be explained by dielectrophoresis. Due to the roughness of the surface (for example the holes visible at the AFM scan in figure 3-2), the electric field will be non-uniform inside the nanoslits, resulting in high local electric field gradients. Since the dielectrophoretic force is proportional to the squared applied field gradient, charged molecules can be trapped at such places ${ }^{29}$. The higher the electric field, the more difficult it is to escape out of these traps. The trapping of DNA molecules by dielectrophoresis has been reported in several papers ${ }^{29-32}$. Ajdari and Prost ${ }^{28}$ predicted an 


\section{Field Dependent DNA Mobility}

exponential dependence of the trapping time on the electric field strength. A combination of both steric trapping and dielectrophoresis at sites of surface roughness is also possible. Using the approach presented in figures 3-1 and 3-2, we calculated the mobility at different applied fields from observed $\lambda$-DNA molecule movement.

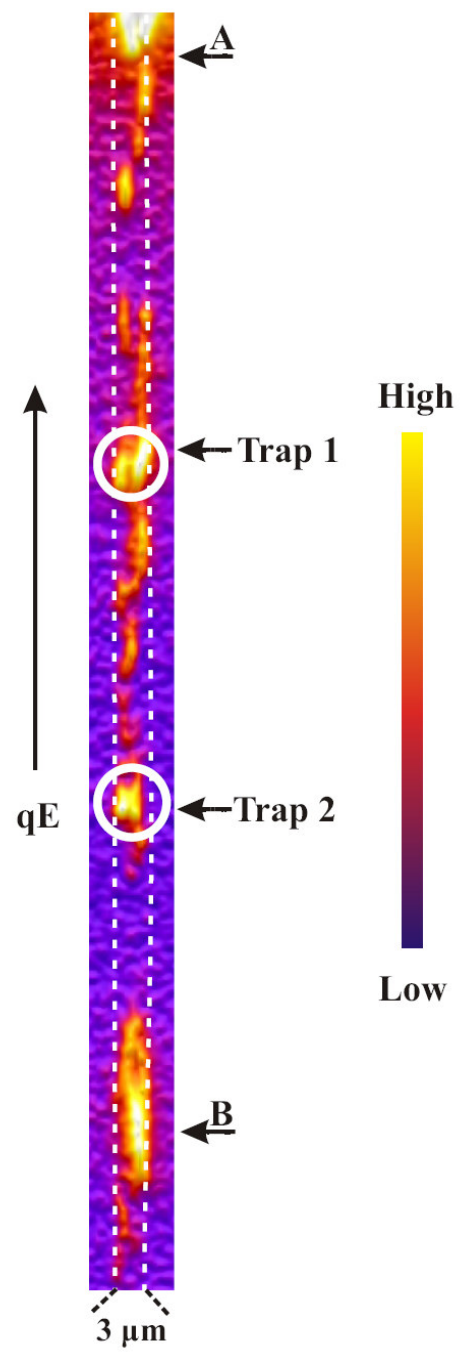

Figure 3-3. By superimposing all the frames of one movie, the pathways of the DNA molecules can be traced inside a particular part of a nanoslit. Again, the same movie as in figure 3-1 is taken. A is the exit of the nanoslit, whereas at the location of $\mathrm{B}$ molecules remained permanently trapped during this experiment. The white circles (trap 1 and 2) represent two locations inside the nanoslit where the molecules were transiently trapped and then continued moving. Interestingly, the molecules seem to follow preferred pathways inside these nanoslits. 
Chapter 3

For the fluent movements at low applied fields, a direct calculation of the mobility was possible, while for the intermittent movements two different values of the mobility were calculated, namely an overall mobility which included the trapping times and a mobility for the phases of movement alone. It is important to mention that the limited frame rate $(5 \mathrm{~Hz})$ will cause a certain underestimation of the mobility determined for the movement phases during intermittent movement. Another source of error for the mobility data is that a fraction of molecules was permanently trapped during the entire duration of the experiment (typically 60 s.). This fraction increased with increasing applied electric field. These molecules were not included in the data used to calculate the mobility. The true average mobility will for this reason be even lower than presented here. Figure 3-4 displays an overview of the mobilities measured at all applied fields. We will now compare our results with the free solution mobility reported for DNA and the mobilities of DNA inside nanoslits of comparable dimensions as reported by Cross and coworkers ${ }^{23}$.

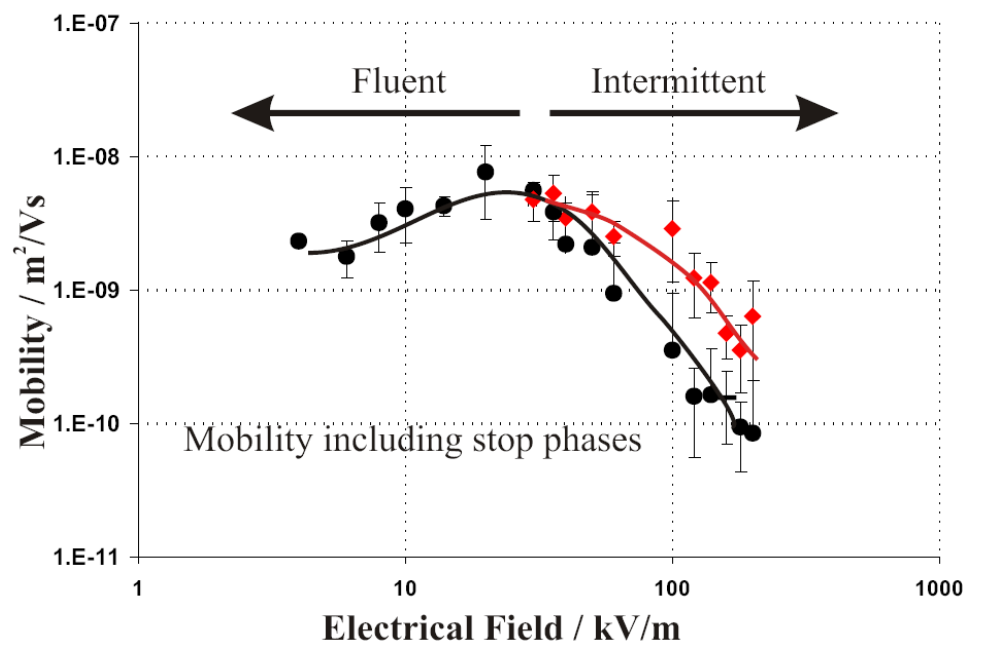

Figure 3-4 The mobilities of $\lambda$-DNA molecules in different applied fields. The mobility of 15 or more $\lambda$-DNA molecules was calculated for each data point. At fields lower than $30 \mathrm{kV} / \mathrm{m}$ the movement of the molecules is fluent, whereas above $30 \mathrm{kV} / \mathrm{m}$ the movement is intermittent. For the intermittent movements we have calculated two different mobilities. First an overall mobility in which we include both periods in which the molecule moved and was trapped, and second a mobility calculated only from the movement phases (red line). The lines drawn are only an indication for the eye. Error bars represent the standard deviation.

The experiments in Cross' investigation were performed at low electric fields $(2-6 \mathrm{kV} / \mathrm{m})$ in (partly) $19 \mathrm{~nm}$ high fused silica nanoslits. The free solution mobility they found was $1.1^{*} 10^{-8} \mathrm{~m}^{2} / \mathrm{Vs}$ (8 $\mathrm{kb}$ DNA molecules). Cross et al. presented a theoretical calculation based on a simple friction model to explain the observed mobility. Applying this calculation to our (longer) $\lambda$-DNA, the calculated mobility in the nanoslit is $7.2 * 10^{-9}$ $\mathrm{m}^{2} / \mathrm{Vs}$. In the electric fields below $30 \mathrm{kV} / \mathrm{m}$ where fluent movement occurred, the 


\section{Field Dependent DNA Mobility}

mobility we found was about a factor of 2 smaller than this value ${ }^{23}$. Since the fields we applied are comparable, similar mobilities would be expected. Possible explanations for the low mobility we found can be a difference in surface roughness as a result of the fabrication or material method used, the fact that Cross' equation cannot be extrapolated to longer DNA lengths. Two other possible explanations revolve around the fact that Cross used a slightly lower PVP concentration than we did (2\% instead of $2.5 \%)$ which could cause a different level of residual EOF or less friction with PVP.

Above $30 \mathrm{kV} / \mathrm{m}$, the mobility decreases rapidly with electric field and a transition from fluent movement to intermittent movement is observed. The mobility calculated from the movement phases decreases to $5 \%$ of the free solution mobility at $200 \mathrm{kV} / \mathrm{m}$, whereas the overall mobility in which the trapping times are included is only $1 \%$ of the free solution mobility. To our knowledge, such a strong field dependent mobility has not been reported before in literature.

To investigate the field dependent trapping inside the nanoslits in more detail, frames of experiments at different applied electric fields were superimposed in the same way as for figure 3-3 (figure 3-5). The experiment shown started at high electric field to pull the $\lambda$ DNA molecules into the nanoslits. In this figure 3-5, A indicates the exit of the nanoslit, whereas B and C show examples of traps. Clearly in fields of $50 \mathrm{kV} / \mathrm{m}$ and above, more $\lambda$ DNA molecules are trapped and at more locations. When the field subsequently was decreased, molecules were less and less trapped. In a few traps however, $\lambda$-DNA molecules remained trapped also at the low voltages. Detailed pictures of DNA in trap B and C at 10 $\mathrm{kV} / \mathrm{m}$ show hook-shaped $\lambda$-DNA molecules, which were not able to escape out of these traps during the entire experiment (and thus not included in the calculations). When figure $3-5 \mathrm{~B}$ and $\mathrm{C}$ are compared, it can be seen that the lengths of these $\lambda$-DNA molecules differ on the eye. However, it was observed that trapped molecules were able to stretch during the experiments and therefore it is difficult to estimate the length of these trapped $\lambda$-DNA molecules. Next to the stretching, it was seen that the intensity of the fluorescent signal could vary during stretching events. Important to mention is that during the experiments we found no proof of breakage of $\lambda$-DNA molecules. To check the molecules on their lengths afterwards is complicated. The final concentration in the waste vial is too low, for a direct control on an agarose gel for example. The extra steps necessary to check the lengths in this way, could also break these molecules. At applied electric fields of higher than 30 $\mathrm{kV} / \mathrm{m}$, most $\lambda$-DNA molecules were delayed or trapped at B and C. When an electric field of $10 \mathrm{kV} / \mathrm{m}$ was applied, $\lambda$-DNA molecules were not delayed or trapped at $\mathrm{B}$ and $\mathrm{C}$ anymore, but were able to flow fluently through the entire part of the nanoslit, albeit on average with some delay. Besides the field dependence of DNA movement in the region of intermittent movement it was also investigated how the field is influencing the mobility in the region of fluent movement. From $4-30 \mathrm{kV} / \mathrm{m}$ an increase in mobility is found with increasing DC field. This could be explained with the biased reptation model as presented by Slater and Noolandi in 1985. In chapter 2 (section 2.4.2.2) biased reptation is explained. In brief, the DNA moves through the gel like a snake in a tube (the "reptation" part) and the electric force causes preferential motion in the direction of the electric field (the 
Chapter 3

"biased" part). The preferential pathways we find inside the nanoslits can be seen as the "tubes" of the DNA molecules.
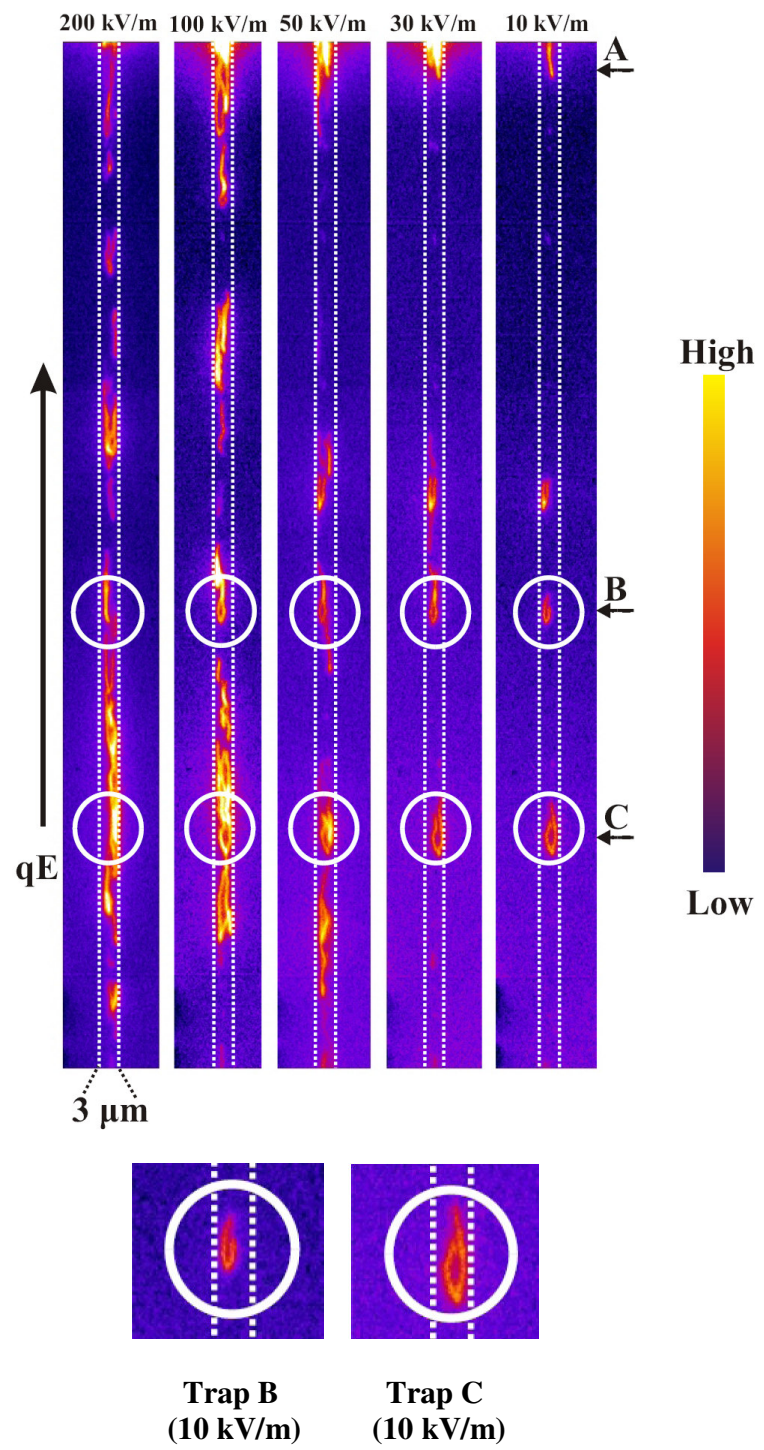

Figure 3-5 By superimposing 300 consecutive frames of experiments at different applied electric field strength, the trapping of $\lambda$-DNA in a nanoslit can be studied. A indicates the exit of the nanoslit. The white circles (B and C) indicate examples of traps. At $10 \mathrm{kV} / \mathrm{m}$ some DNA molecules were delayed at these points, but at electric fields of $30 \mathrm{kV} / \mathrm{m}$ and above most $\lambda$-DNA was temporarily delayed or trapped at these locations. 


\section{Field Dependent DNA Mobility}

As long as the molecule is not fully oriented in the direction of the applied DC field inside the nanoslit, it will increase in velocity (and thus mobility) with increasing DC field strength. This could explain the first part of the mobility plot for the $20 \mathrm{~nm}$ high nanoslits in figure 3-4, where the mobility is increasing. At DC fields above $30 \mathrm{kV} / \mathrm{m}$, the intermittent movements prevent the molecules from the fully orienting resulting after being trapped.
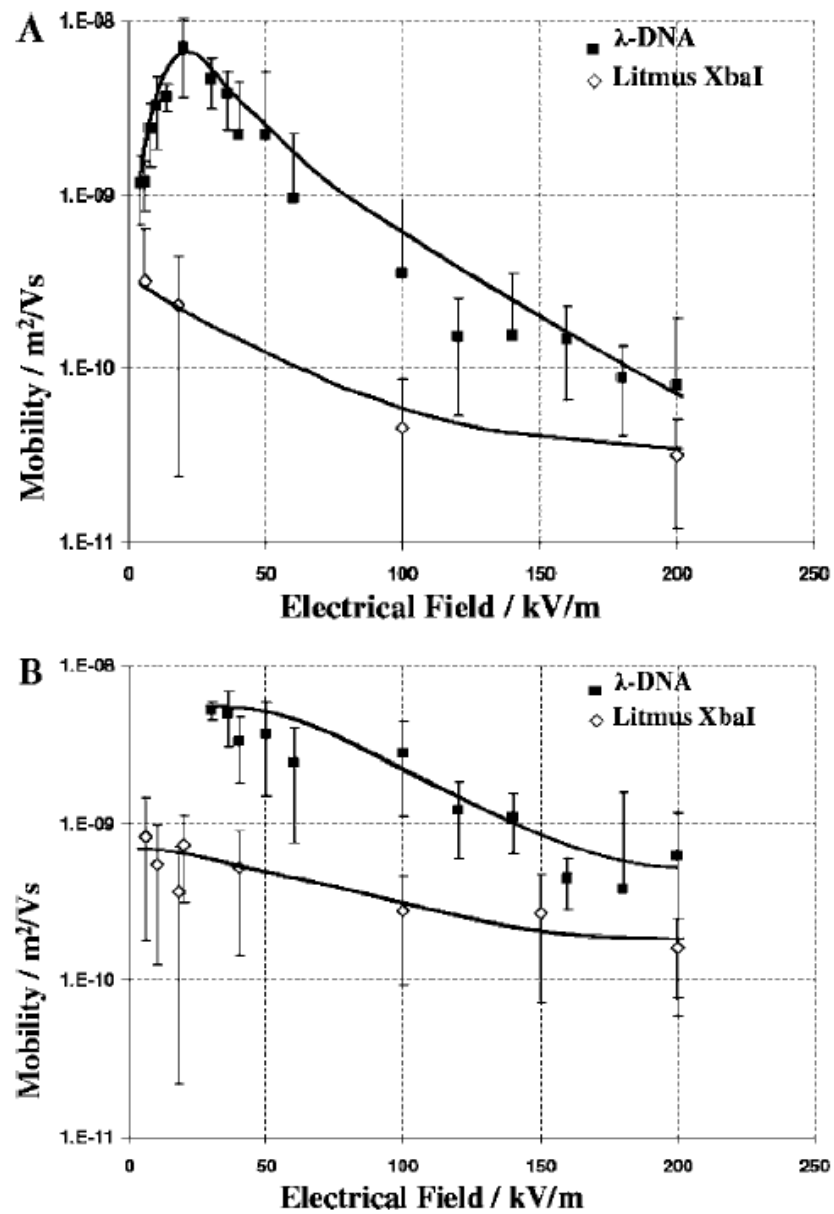

Figure 3-6 A) The overall mobility of $\lambda$-DNA and Litmus DNA (XbaI digested). The preliminary data of Litmus DNA indicate that the overall mobility is much lower than for $\lambda$-DNA. B) The mobility calculated only from the moving phases between trapping events. Litmus DNA (XbaI digested) moves in an intermittent way at electric fields between 6 and $30 \mathrm{kV} / \mathrm{m}$, where $\lambda$-DNA molecules move fluently through the nanoslits. The lines drawn are only an indication for the eye. Error bars represent the standard deviation. 
Chapter 3

It was investigated whether the observed field-dependent behavior was different for another type of DNA molecule namely Litmus DNA (digested with XbaI, $2.8 \mathrm{kbp}$ ). Figure 3-6 A and B compare the mobility of $\lambda$-DNA with preliminary results for the mobility of Litmus DNA (XbaI digested). Figure 3-6A shows that the overall mobility for $\lambda$-DNA is indeed considerably higher than the overall mobility of Litmus DNA. This size-dependent mobility could offer a basis for a length-dependent DNA separation. Figure 3-6B shows that also the mobilities during the moving phases differ. The mobility of $\lambda$-DNA is higher at all investigated fields than the mobility of Litmus DNA. This difference is as yet unexplained, but possibly transient trapping of parts of the molecule play a role. We found that even at the low applied field of $6 \mathrm{kV} / \mathrm{m}$ still intermittent Litmus DNA movement was observed. Thus, the shorter Litmus DNA is still trapped at electric fields where the longer $\lambda$-DNA moves in a fluent way through the nanoslits. This behavior could possibly be explained by dielectrophoretic trapping, if it is assumed that the dielectrophoretic traps are smaller than the size of $\lambda$-DNA but closer to the dimensions of Litmus DNA. In that case the larger $\lambda$-DNA will be able to escape more easily than the Litmus DNA at high applied electric field since a smaller part of the $\lambda$-DNA molecule is trapped by dielectrophoresis than the Litmus DNA. We investigated this phenomenon in more detail in chapter 4, since differences in field-dependent mobility could enable separation of DNA. Especially, the ease with which an applied field can be changed would offer a great advantage for separations, offering a convenient control parameter.

\subsection{Conclusion}

To our knowledge, we reported for the first time a field dependent mobility of $\lambda$-DNA molecules in nanoslits at applied high electric fields. At low electric fields (below 30 $\mathrm{kV} / \mathrm{m}$ ) a fluent movement was seen with a mobility a factor of two lower than the mobility measured in nanoslits of comparable dimensions by Cross and coworkers ${ }^{23}$. At electric fields of $30 \mathrm{kV} / \mathrm{m}$ and above, an intermittent movement and strongly field-dependent trapping of the DNA was observed, resulting in a strongly reduced mobility at high fields. Preliminary results show a difference in trapping behavior of $\lambda$-DNA and Litmus DNA at low applied electric fields which might be explained by dielectrophoretic trapping and/or mechanical trapping. Together with the recently published size dependent DNA mobility in nanochannels of Cross and coworkers ${ }^{23}$, the observed behavior can open the door for the development of new gel free separation devices.

\section{Acknowledgements}

We thank Kevin Dorfman and Jean Louis Viovy for the inspiring discussions, Wouter Sparreboom for the support with the electric potential measurements and Martin Siekman for the help with the AFM measurements. Financial support of NanoNed and Nano2Life is gratefully acknowledged. Jonas Tegenfeldt's work is financed by the Swedish Research Council, grant 2002-5972. 


\section{Field Dependent DNA Mobility}

\subsection{References}

1. W. Reisner, K. J. Morton, R. Riehn, Y. M. Wang, Z. Yu, M. Rosen, J. C. Sturm, S. Y. Chou, E. Frey, R. Austin, Phys. Rev. Let., 2005, 94, 196101.

2. J. O. Tegenfeldt, C. Prinz, H. Cao, S. Chou, W. W. Reisner, R. Riehn, Y. M. Wang, E. C. Cox, J. C. Sturm, P. Silberzan, R. H. Austin, Proc. Nat. Acad. Sci. USA, 2004, 101, 30, $10979-10983$.

3. A. Balducci, P. Mao, J. Han, P. S. Doyle, Macromolecules, 2006, 39, 6273-6281.

4. P.K. Lin, C. C. Fu, Y. L. Chen, Y. R. Chen, P. K. Wei, C. H. Kuan, W. S. Fann, Phys. Rev. E, 2007, 76, 011806.

5. J. T. Mannion, C. H. Reccius, J. D. Cross, H. G. Craighead, Biophys. J., 2006, 90, 4538- 4545.

6. C. H. Reccius, J. T. Mannion, J. D. Cross, H. G. Craighead, Phys. Rev. Let., 2005, 95, 268101.

7. C. C. Hsieh, A. Balducci, P. S. Doyle, Macromolecules, 2007, 40, 5196-5205.

8. L.C. Campbell, M. J. Wilkinson, A. Manz, P. Camilleri, C. J. Humphreys, Lab on a Chip, 2004, 4, 225-229.

9. J. Fu, P. Mao, Appl. Phys. Let., 2005, 87, 263902.

10. R. Riehn, R. H. Austin, J. C. Sturm, Nano Lett., 2006, 6, 1973-1976.

11. Y. M. Wang, J. O. Tegenfeldt, J. Sturm, R. H. Austin, Nanotechnology, 2005, 16, 1993-1999.

12. Y. M. Wang, J. O. Tegenfeldt, W. Reisner, R. Riehn, X. J. Guan, I. Golding, E. C. Cox, J. Sturm, R. H. Austin, Proc. Nat. Acad. Sci. USA, 2005, 102, 28, 9796-9801.

13. J. L. Viovy, Rev. Mod. Phys., 2000, Vol. 72, No. 3, 813-872.

14. G. W. Slater, Electrophoresis, 2002, 23, 3791-3816.

15. J. C. T. Eijkel, A. van den Berg, A., Electrophoresis, 2006, 27, 677-685.

16. J. C. T. Eijkel, A. van den Berg, A., Lab on a Chip, 2006, 6, 1, 19-23.

17. J.O. Tegenfeldt, C. Prinz, H. Cao, R. L. Huang, R. H. Austin, S. Y. Chou, S. Y, E. C. Cox, J. C. Sturm, Anal. Bioanal. Chem., 2004, 378, 1678-1692.

18. F. Brochard, Le Journal de Physique 1977, 38, 1285-1291.

19. F. Brochard, P. G. de Gennes, J. Chem. Phys., 1977, 67, 52-56.

20. T. Odijk, Macromolecules 1983, 16, 1340-1344.

21. C. H. Reccius, J. T. Mannion, J. D. Cross, H. G. Craighead, Phys. Rev. Let., 2005, 95, 268101.

22. M. Krishnan, I. Mönch, P. Schwille, Nano Lett., 2007, 7, 1270-1275.

23. J. D. Cross, E. A. Strychalski, H. G. Craighead, J. Appl. Phys. 2007, 102, 024701

24. S. Pennathur, F. Baldessari, J. G. Santiago, M. G. Kattah, J. B. Steinman, P. J. Utz, Anal. Chem., 2007, 79, 8316-8322.

25. J. Regtmeier, T. T. Duong, R. Eichhorn, D. Anselmetti, A. Ros, Anal. Chem., 2007, 79, 3925-32.

26. C. L. Asbury, A. H. Diercks, G. van den Engh, Electrophoresis, 2002, 23, 2658-2666.

27. M. G. Gauthier, G. W. Slater, J. Chem. Phys. 2002, 117, 6745-6756.

28. A. Ajdari, J. Prost, Proc. Nat. Acad. Sci, 1991, 88, 4468-4471.

29. C. F. Chou, J. O. Tegenfeldt, O. Bakajin, S. S. Chan, E. C. Cox, N. Darnton, T. Duke, 


\section{Chapter 3}

R. H. Austin, Biophys. J. 2002, 83, 2170-2179.

30. S. Tuukkanen, J. J. Toppari, A. Kuzyk, L. Hirviniemi, T. Hytönen, T., Ihalainen, P. Törmä, Nano Lett. 2006, 6, 1339-1343.

31. D. J. Bakewell, H Morgan, IEEE Trans. Nanobiosci. 2006, 5, 1-8.

32. S. Nagahiro, S. Kawano, H., Kotera, Phys. Rev. 2007, E75, 011902.

33. G.W. Slater, J. Noolandi, 1985, Phys. Rev. Lett., 55, 151579 - 1582. 
Filename: $\quad 3 \_$New Header_DC Field dependent DNA mobility_For_WPS_Final

Directory: $\quad$ C:Locuments and SettingsiGeorgette.UTWENTEA174A94\Desktop

Template: $\quad$ C:LDocuments and SettingsiGeorgette.UTWENTEA174A94\Application DatalMicrosoft \Templates\Normal.dot

Title:

Subject:

Author:

Georgette

Keywords:

Comments:

Creation Date: $\quad$ 10/8/2009 10:47:00 AM

Change Number: 2

Last Saved On: $\quad$ 10/8/2009 10:47:00 AM

Last Saved By: Georgette

Total Editing Time: 5 Minutes

Last Printed On: $\quad$ 10/8/2009 11:12:00 AM

As of Last Complete Printing

Number of Pages: 12

Number of Words: $\quad 3,265$ (approx.)

Number of Characters: 18,616 (approx.) 


\section{4}

\section{AC/DC Field Dependent DNA Mobility in 20 nm High Nanoslits}

In this chapter, investigations on the presence of dielectrophoretic traps in the $20 \mathrm{~nm}$ high nanoslits are presented. For this purpose, AC electric fields $(10-200 \mathrm{kV} / \mathrm{m})$ with frequencies of $100 \mathrm{~Hz}$ and $1 \mathrm{kHz}$ are superimposed on DC electric fields of $8-200 \mathrm{kV} / \mathrm{m}$, and the electrokinetic transport behaviour of the $\lambda$-DNA molecules investigated. For all investigated DC fields below $20 \mathrm{kV} / \mathrm{m}$ and all superimposed AC fields $(1 \mathrm{kHz})$, a significant increase in mobility is found, which would not be expected in case of the presence of a dielectrophoretic trapping mechanism. This increase in mobility can possibly be explained by an increase in the orientation of the molecule in the direction of the field with the biased reptation model, and is then comparable to results of investigations obtained in gel electrophoresis. The measured mobilities at electric DC fields above 20 $\mathrm{kV} / \mathrm{m}(100 \mathrm{~Hz}$ and $1 \mathrm{kHz})$ provide a complex picture and next to a few exceptions, which need to be further investigated, no proof for the presence dielectrophoretic trapping mechanism could be found.

Manuscript in preparation: O. Castillo-Fernandez, G.B. Salieb-Beugelaar, J.W. van Nieuwkasteele, J.G. Bomer, M. Arundell, J. Samitier ${ }^{1}$, A. van den Berg, J.C.T. Eijkel. 


\subsection{Introduction}

In chapter 3 we saw that when DC electric fields up to $30 \mathrm{kV} / \mathrm{m}$ were applied, the molecules move fluently through the slits with an increasing mobility. At higher electric fields, the DNA molecules move intermittently ("Stop and Go"), resulting in a strongly

reduced mobility ${ }^{1,2}$. We proposed that this behavior could be explained by mechanical and/or field-induced dielectrophoretic DNA trapping in relation to the surface roughness in the nanoslits. The observation of preferential pathways and trapping sites of the $\lambda$-DNA molecules in the nanoslits supported this hypothesis. As we saw in Chapter 2, DNA molecules are negatively charged due to the phosphate groups outside their helix, causing transport by DC electric fields. When no field is applied, the counterions shield the charges at the surface symmetrically as presented in figure 4-1A. As soon as an electric field is applied, the counterions will move to the opposite charged pole (figure 4-1B). This results in a polarization of the DNA molecule.

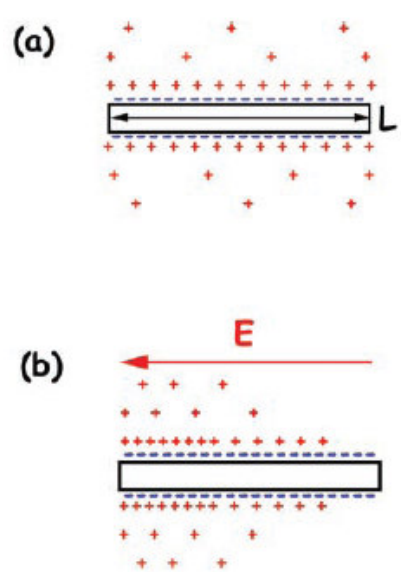

Figure 4-1. The bar in this figure represents a negatively charged DNA molecule in a solution shielded by its positive counterions (a). No electric field is applied; (b). As soon as an electric field is applied, the counterions will move to the opposite charged pole. This will lead to a polarization of the DNA molecule as presented in (b) and a net surface charge of the density along the length of the molecule.

The resulting dipole moment is caused by the movement of the counter ions and therefore depends of the applied electric field, the time this field is applied and the length of the DNA. Returning to the nanoslits, if the electric DC field inside these channels would be non-uniform as a result of the surface, then the density of the electric field lines inside these nanoslits would also be non-uniform. At places with a high local field gradient, DNA molecules could then be trapped (dielectrophoretic traps). Dielectrophoretic trapping of $\lambda$ DNA molecules was for example studied by Asbury and van den Engh ${ }^{5}$. In their investigation, the molecules could be trapped in AC fields using a range of frequencies of 1 $\mathrm{Hz}-1 \mathrm{kHz}$. Trapping at higher frequencies was possible, however higher AC fields were 


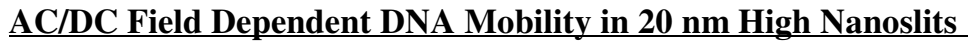

necessary. Another very interesting investigation was done by $\mathrm{Chou}^{6}$, who showed the dielectrophoretic trapping of DNA molecules with lengths of $368 \mathrm{bp}$ and $39.9 \mathrm{kbp}$ at channel constrictions by using AC fields with frequencies till $1 \mathrm{kHz}$. Especially at $100 \mathrm{~Hz}$ and an AC field of $200 \mathrm{kV} / \mathrm{m}$, the $\lambda$-DNA molecules were trapped so tightly in the constrictions, that their data analysis was not possible. In the study presented here, we investigate the hypothesis of dielectrophoretic trapping of the $\lambda$-DNA molecules in the nanochannels at high applied electric DC fields. In addition to the applied DC fields we therefore now superimpose an AC field in order to increase the field line density inside the nanoslits and thereby the dielectrophoretic force without increasing the (DC determined) electrophoretic force.

\subsection{Experimental protocol}

\subsubsection{The nanoslit device}

The surface roughness of the batch of nanoslit devices was determined using an AFM and we found that the rms value is $\sim 1 \mathrm{~nm}$. We found holes in the surface with a depth until $\sim 8$ $\mathrm{nm}$. This is comparable with AFM scans made of the nanoslit surfaces of the devices used in the investigation presented in Chapter 3. The height of the nanoslits was confirmed to be $20 \mathrm{~nm}$.

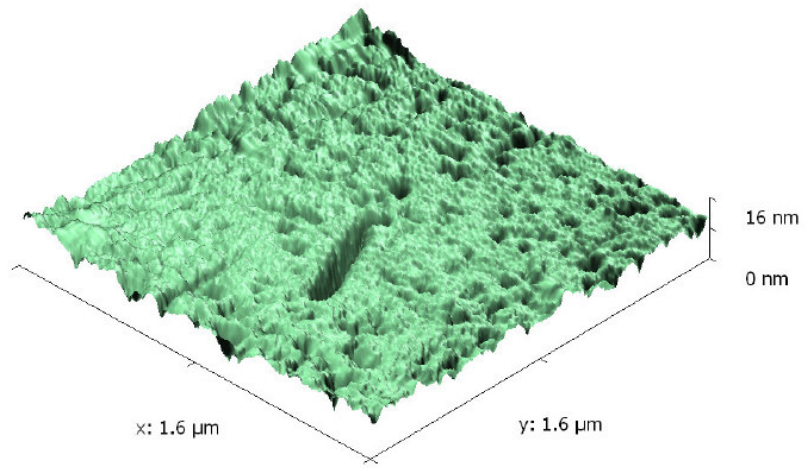

Figure 4-2. An example of the surface roughness of the $20 \mathrm{~nm}$ high nanoslits as measured by AFM and using tips with a radius of $2 \mathrm{~nm}$. The rms value is $0.8-1.4 \mathrm{~nm}$ which is comparable with the rms value in our previous investigation (Chapter 3$)^{8}$. Also here holes occur in the surface with a depth down to $8 \mathrm{~nm}$.

\subsubsection{Electric setup}

The scheme of the experimental setup used is shown in figure 4-3. The AC and DC electric field generation was achieved by connecting a standard function generator (Agilent 33220A) with a High Voltage Signal Amplifier (Treck 677B). The Amplifier setup allows 


\section{Chapter 4}

200 times amplification of the signal. The effective work range of the amplifier is up to a frequency of $1.2 \mathrm{kHz}$. We calibrated the amplifier using an oscilloscope. The bias of the $\mathrm{AC}$ instruments on the applied DC offset was determined and corrected. In the DC and AC range where the experiments were performed, the error after correction in the DC applied field is $\pm 1 \mathrm{kV} / \mathrm{m}$ and the error in the AC applied field is $\pm 0.02 \mathrm{kV} / \mathrm{m}$. DC electric fields between 8 and $200 \mathrm{kV} / \mathrm{m}$ were applied and AC fields between $10-200 \mathrm{kV}$ with frequencies of $100 \mathrm{~Hz}$ and $1 \mathrm{kHz}$ were superimposed on these DC fields.

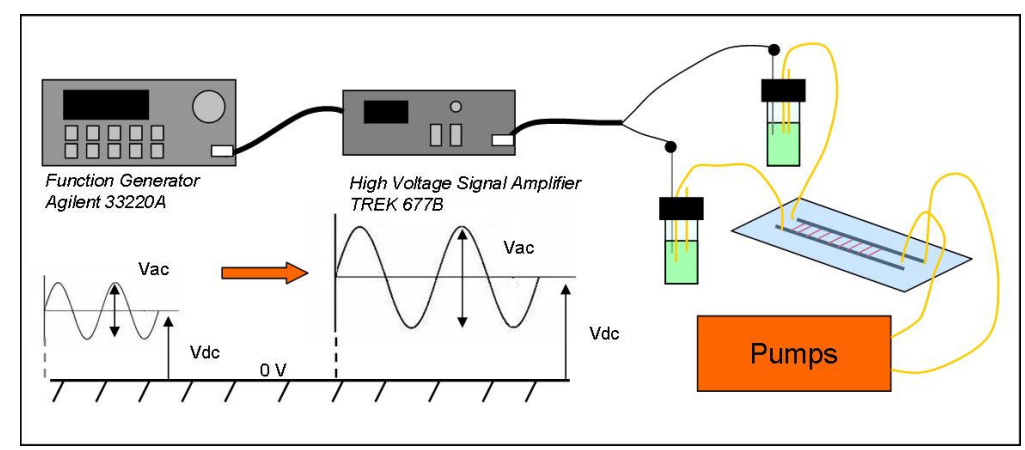

Figure 4-3. The experimental setup, showing the electric equipment connected to the nanoslit device via electrode reservoirs. The device consists of two microchannels connected by a parallel array of 100 nanoslits. The two microchannels are connected via capillaries to a syringe pump at one side and at the other side to the buffer vials in which we put the Pt electrodes.

DNA translocation experiments were carried out at the applied AC/DC fields. Data were pooled and the analysis was performed as described in chapter $3^{2}$. In brief, $\lambda$-DNA molecules were traced in successive video frames and from these data the velocities and mobilities were calculated. The used frame rate was $22 \mathrm{~Hz}$, which is roughly 5 times faster than the frame rate of $5 \mathrm{~Hz}$ in the previous investigation (chapter 3$)^{2}$.

\subsection{Results and discussion}

In this investigation we found the same two types of movement of the $\lambda$-DNA molecules as in our previous investigation (Chapter 3). The first type is the fluent movement where the molecules follow their way through the nanoslits without stopping. The second type is the intermittent movement where the molecules move in a stop and go fashion, stopping at particular places. As a result of these two types of movement, the transport behavior of the $\lambda$-DNA molecules was investigated in two different ways as described in the previous chapter ${ }^{1}$. For the fluent movements at low applied fields, a direct calculation of the mobility was possible, while for the intermittent movements two different values of the mobility were calculated, namely an overall mobility which included the stopping times and a mobility for the phases of movement alone or "go phase" mobility. At each applied DC electric field, a data group of the measured mobilities was made for each applied AC 


\section{AC/DC Field Dependent DNA Mobility in $20 \mathrm{~nm}$ High Nanoslits}

field between $0-200 \mathrm{kV} / \mathrm{m}$. From these data groups, the median and the standard deviation were calculated and plotted in the graphs presented below (figure 4-4 - 4-7). At each applied DC field, the difference between the applied AC fields was statistically tested. The Kruskal-Wallis one way of variance on ranks test (ANOVA) was used for this purpose since the distribution of the data was not always normal and the number of data points for each data group was not equal ${ }^{7}$. If the difference was significant, a pairwise comparison of the data groups was performed to isolate the significantly different data groups. For this pairwise comparison, Dunn's method was used, which allows non-normal distribution and unequal numbers of data points of the data groups ${ }^{8}$.

We will first present an overview of all significant data in table 1 and discuss this briefly whereafter a detailed discussion of the results will follow. In table $1 \mathrm{~A}$ (the $1 \mathrm{kHz}$ data) it can be seen that in all cases the significant data show an increase in mobility. This increase in mobility is in contradiction with a possible presence of a dielectrophoretic trapping mechanism. It would namely be expected that the higher density of the electric field lines resulting from the superimposition of the AC fields onto the DC fields, would decrease the mobility if dielectrophoretic trapping occurred. In table $1 \mathrm{~B}$ (the $100 \mathrm{~Hz}$ data), it can be seen that the measured mobilities are conflicting. For the DC fields of $10 \mathrm{kV} / \mathrm{m}$ we see an increase and decrease in the mobility for the low and high superimposed AC field strength respectively. For the DC fields of $20 \mathrm{kV} / \mathrm{m}$ we find a decrease and an increase, for the low and high superimposed AC fields. This is a contradiction and therefore not pointing into the direction of dielectrophoretic trapping. More details regarding type of movement (intermittent or fluent) will be discussed below.
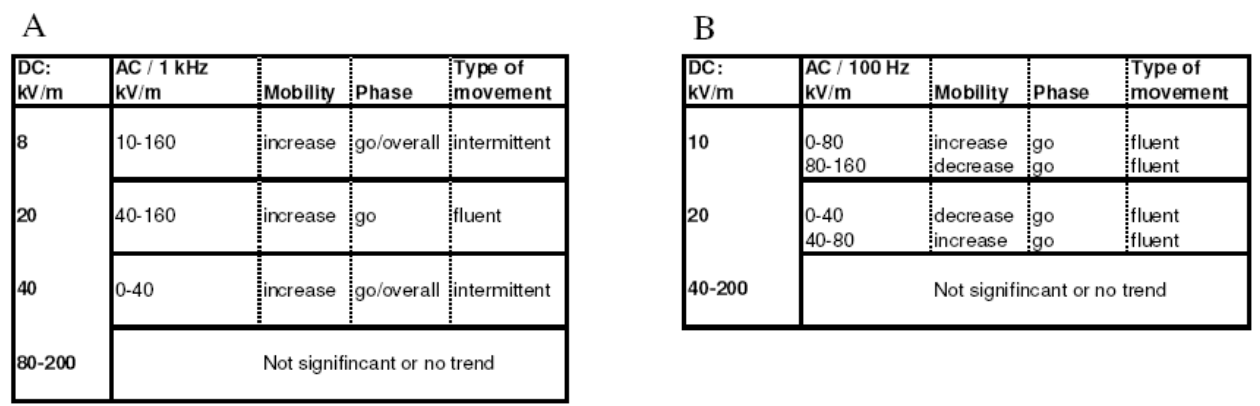

Table 1. In A an overview of the significant results provided when $\mathrm{AC}$ fields of $1 \mathrm{kHz}$ are superimposed into DC fields. It can be seen that all significant results are an increase in mobility. In $\mathrm{B}$, the mobilities measured with the superimposition of $\mathrm{AC}$ fields with a frequency of $100 \mathrm{~Hz}$ are presented. It can be seen that at an applied DC field of $10 \mathrm{kV} / \mathrm{m}$ the mobility increases in the low AC fields and decreases in the higher AC fields. This could point into the direction of a dielectrophoretic trapping mechanism. When regarding the DC fields of $20 \mathrm{kV} / \mathrm{m}$, we see first a decrease in mobility in the low AC fields, where after the mobility is increased in the higher AC fields.

First, the overall mobilities will now be discussed in more detail, followed by the "go phase" mobilities. It is important to mention that when only fluent movements occur, both mobilities will be equal and will appear in both graphs. 


\section{Chapter 4}

A

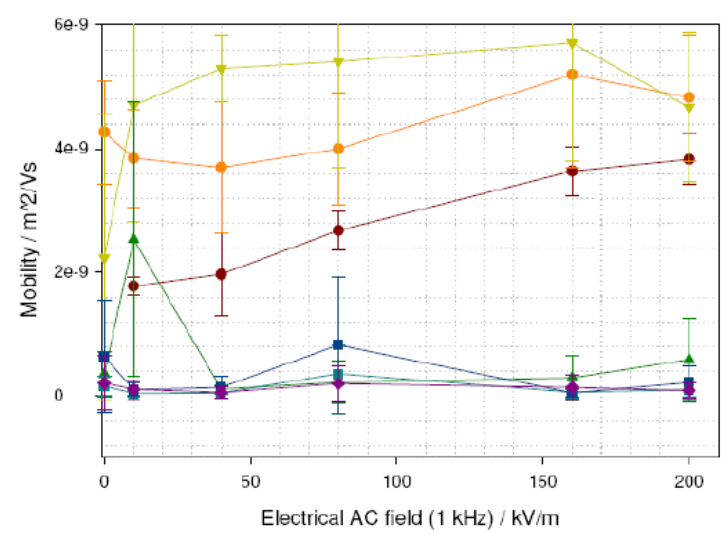

DC:

$-8 \mathrm{kV} / \mathrm{m}$

$\longrightarrow 20 \mathrm{kV} / \mathrm{m}$
$\because-40 \mathrm{kV} / \mathrm{m}$

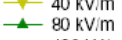

$=120 \mathrm{kV} / \mathrm{m}$

$-160 \mathrm{kV} / \mathrm{m}$

$-200 \mathrm{kV} / \mathrm{m}$

Electrical AC field $(1 \mathrm{kHz}) / \mathrm{kV} / \mathrm{m}$

B

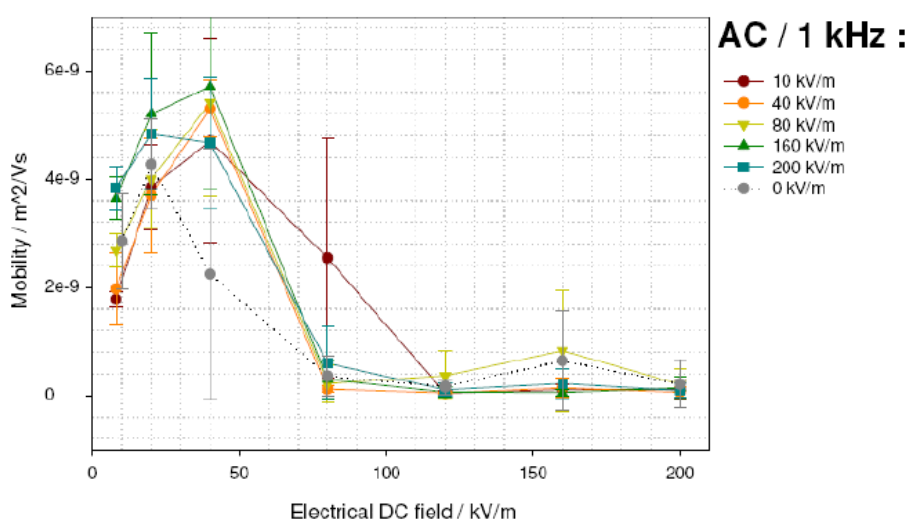

Figure 4-4. A presents the overall mobilities of the $\lambda$-DNA molecules versus the AC fields of $1 \mathrm{kHz}$ superimposed onto DC fields of various strengths. As can be seen in the graph at DC fields below 40 $\mathrm{kV} / \mathrm{m}$ the AC field increases the mobility of the molecules. For example the plot of $8 \mathrm{kV} / \mathrm{m}$ in DC shows a significant increase of the mobility for the applied AC fields $80 \mathrm{kV} / \mathrm{m}(\mathrm{p}<0.05)$. B presents the overall mobilities at DC fields of various strengths. As can be concluded from both A and B, at fields of 8 and $20 \mathrm{kV} / \mathrm{m}$ in DC the mobility increases with increasing amplitude. Error bars represent the standard deviation

\subsubsection{A Overall mobilities, $1 \mathrm{kHz}$ AC}

The results of the overall mobilities of the $\lambda$-DNA molecules versus the DC fields at various applied $\mathrm{AC}$ fields of $1 \mathrm{kHz}$ are presented in figure 4-4 A and $\mathrm{B}$. In these graphs, the applied AC field of $0 \mathrm{kV} / \mathrm{m}$ represents the mobility of the $\lambda$-DNA molecules when only a DC field is applied. In A, it can be seen that the mobilities clearly increase when AC fields 


\section{AC/DC Field Dependent DNA Mobility in $20 \mathrm{~nm}$ High Nanoslits}

are superimposed until the DC field of $40 \mathrm{kV} / \mathrm{m}$. The statistically significant differences between the data groups are found in this part of the graph. We see a significant increase ( $\mathrm{p}$ $<0.05$ ) of the mobility for the DC electric fields of 8 and $20 \mathrm{kV} / \mathrm{m}$ with increasing AC field strength. Interestingly, the movement of the $\lambda$-DNA molecules at the DC field of $8 \mathrm{kV} / \mathrm{m}$ at all applied AC fields is intermittent, however the trapping times are very short (roughly $5 \%$ ) when comparing to the intermittent movement in the DC fields above $40 \mathrm{kV} / \mathrm{m}$. At an AC field of $200 \mathrm{kV} / \mathrm{m}$, roughly $50 \%$ of the DNA molecules were moving in a fluent manner, while the other DNA molecules were still moving in an intermittent manner. Also, the trapping times decreased with increasing AC field strength. This trapping mechanism is therefore not expected to be dielectrophoretic. At applied DC fields of $40 \mathrm{kV} / \mathrm{m}$ molecules were also trapped at the AC fields of both 10 and $0 \mathrm{kV} / \mathrm{m}$, with similar trapping times. It was indeed expected that the molecules would move in an intermittent way at DC fields of $40 \mathrm{kV} / \mathrm{m}$ without superimposed AC field since this was also found in our previous investigations ${ }^{1}$. The intermittent movement however was not present at AC fields above 10 $\mathrm{kV} / \mathrm{m}$ and DC fields of $40 \mathrm{kV} / \mathrm{m}$. According to the performed statistics, these two datagroups (AC field strength of 10 and $0 \mathrm{kV} / \mathrm{m})$ were significantly different $(\mathrm{p}=0.014)$. When the AC field strength was increased, the $\lambda$-DNA molecules thus were not trapped anymore and moved in a fluent way. The data presented here therefore seem to contradict a dielectrophoretic trapping mechanism. Instead it appeared that the addition of an AC field under a number of circumstances led to an increased mobility or in lesser trapping.

\subsubsection{B Overall mobilities, $100 \mathrm{~Hz}$ AC}

The results of the overall mobilities of the $\lambda$-DNA molecules versus the DC field strength at various applied AC fields $(\mathbf{1 0 0} \mathbf{~ H z})$ are presented in figure 4-5 A and B. Also in these graphs, the applied AC field of $0 \mathrm{kV} / \mathrm{m}$ (dotted curve) represents the mobility of the $\lambda$-DNA molecules when only a DC field is applied. According to the performed statistics, at the lowest DC field of $10 \mathrm{kV} / \mathrm{m}$ the mobility increases significantly when AC fields are superimposed. The mobility plot at the DC field of $10 \mathrm{kV} / \mathrm{m}$ shows this increase of the mobility for applied AC fields until $80 \mathrm{kV} / \mathrm{m}$, whereas the mobility decreases at AC fields of 160 and $200 \mathrm{kV} / \mathrm{m}(\mathrm{p}<0.05)$. The mobility at the AC field of $200 \mathrm{kV} / \mathrm{m}$ is still higher however than the mobility at $10 \mathrm{kV} / \mathrm{m}$ DC alone. Furthermore, we saw that at this DC field, the $\lambda$-DNA molecules moved in a fluent way through the nanoslits at all applied AC fields. The application of AC fields higher than $200 \mathrm{kV} / \mathrm{m}$ was unfortunately beyond the possibilities of our equipment. We also found a significant decrease for the DC fields of 20 and $160 \mathrm{kV} / \mathrm{m}$ when an $\mathrm{AC}$ field of $10 \mathrm{kV} / \mathrm{m}$ was applied with respect to the mobility without AC. At higher AC fields this decrease disappeared, suggesting that it is not caused by dielectrophoresis or that the result was spurious. For the other plots a significant influence of the applied AC field on the mobility of the $\lambda$-DNA molecules was not found. 


\section{Chapter 4}

A

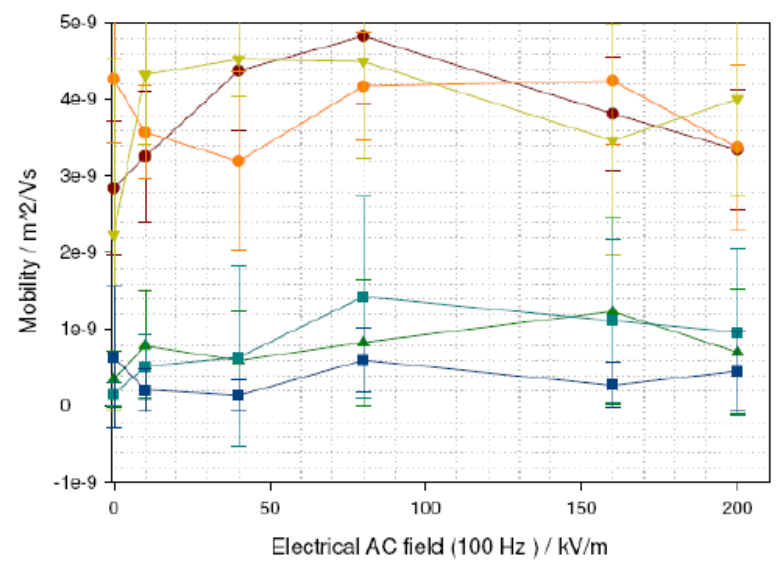

DC:

$-10 \mathrm{kV} / \mathrm{m}$ $\rightarrow 20 \mathrm{kV} / \mathrm{m}$ $-80 \mathrm{kV} / \mathrm{m}$ $\rightarrow-120 \mathrm{kV} / \mathrm{m}$

B

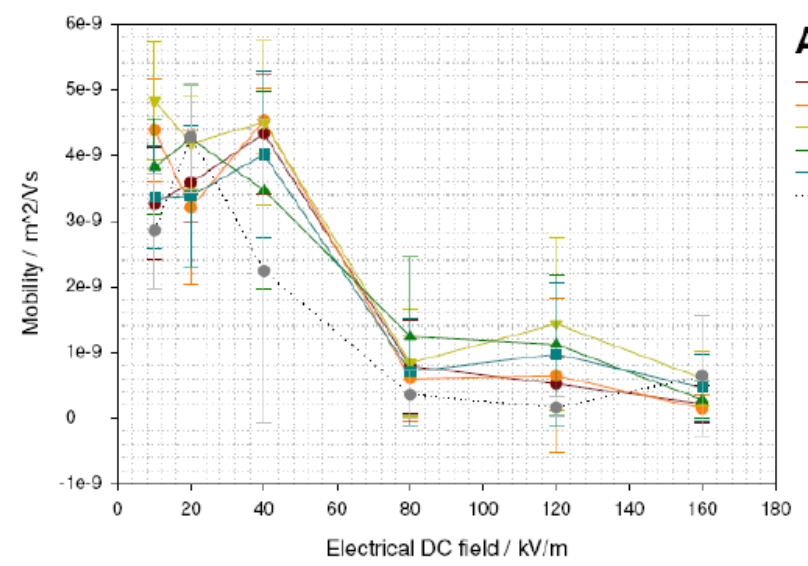

\section{AC / $100 \mathrm{~Hz}$ :}

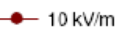

$-40 \mathrm{kV} / \mathrm{m}$

- $80 \mathrm{kV} / \mathrm{m}$

$-160 \mathrm{kV} / \mathrm{m}$

$-100 \mathrm{kV} / \mathrm{m}$

-. $0 \mathrm{kV} / \mathrm{m}$

Figure 4-5. A) The overall mobilities of the $\lambda$-DNA molecules versus the applied AC fields (100 Hz) superimposed at DC fields of various strengths. As can be seen in the graph at the DC field of 10 $\mathrm{kV} / \mathrm{m}$, the AC field does influence the mobility of the molecules. The AC field is increasing the mobility until an AC field strength of $80 \mathrm{kV} / \mathrm{m}$ is applied, whereas at higher AC field strengths the mobility is decreasing. This can also be seen in B where the mobility versus the applied DC field is presented. The significant trend $(\mathrm{p}<0.05)$ of the applied DC field of $10 \mathrm{kV} / \mathrm{m}$ requires further investigation however is beyond the possibilities of our equipment. Any further decrease in mobility until values below an $\mathrm{AC}$ field of $0 \mathrm{kV} / \mathrm{m}$ would suggest the presence of a dielectrophoretic trapping mechanism. Error bars represent the standard deviation.

\subsubsection{Go-phase mobilities, $1 \mathrm{kHz}$}

As mentioned above, when the transport of the $\lambda$-DNA molecules was intermittent, we also calculated the mobility for the movement phases alone ("go phase mobility") which will be 
discussed now. Figure 4-6 A presents the go-mobilities of the $\lambda$-DNA molecules versus the AC field $(1 \mathrm{kHz})$ at various DC field strengths. It can be seen that at the DC field of 40 $\mathrm{kV} / \mathrm{m}$, the mobility increases significantly between the AC field strengths of 0 and 40 $\mathrm{kV} / \mathrm{m}(\mathrm{p}<0.05)$, followed by a significant decrease at the AC fields of 160 and $200 \mathrm{kV} / \mathrm{m}$ $(\mathrm{p}<0.05)$.

A

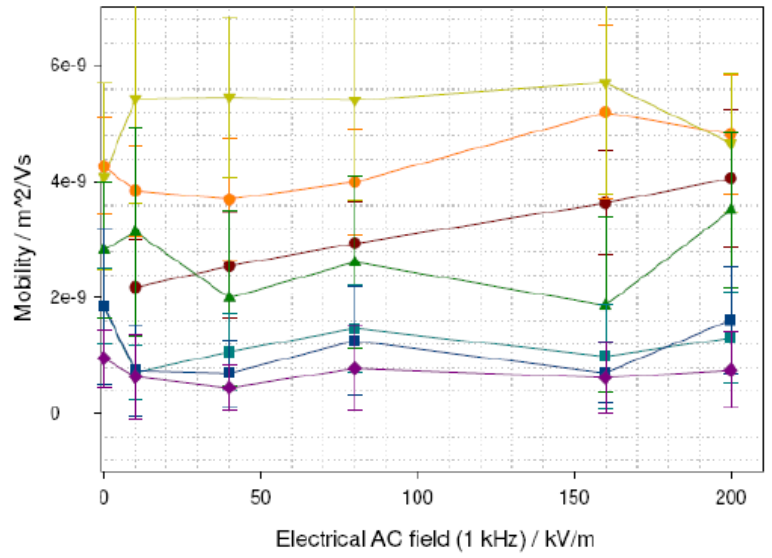

DC:

$\rightarrow-8 \mathrm{kV} / \mathrm{m}$ $-20 \mathrm{kV} / \mathrm{m}$ $-40 \mathrm{kV} / \mathrm{m}$ $-120 \mathrm{kV} / \mathrm{m}$ $-160 \mathrm{kV} / \mathrm{m}$ $\rightarrow 200 \mathrm{kV} / \mathrm{m}$

B

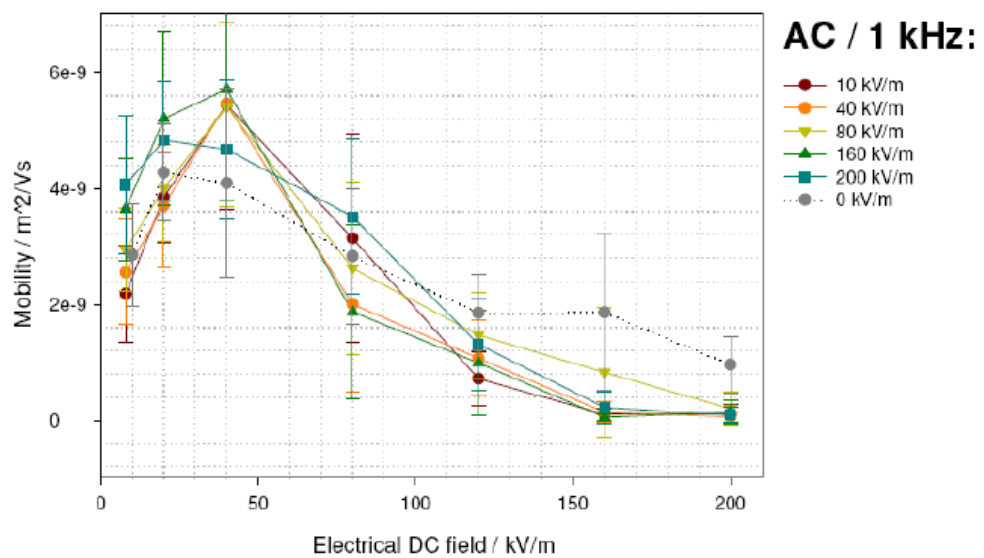

Figure 4-6. A represents the "go" mobility versus the AC field at DC fields of various strengths. At DC fields of 8,20 and $40 \mathrm{kV} / \mathrm{m}$ and several superimposed amplitudes, significant increases in mobilities are found and only one significant decrease in mobility (DC field of $40 \mathrm{kV} / \mathrm{m}$ and AC field of 160 and $200 \mathrm{kV} / \mathrm{m}$ ). B presents the "go" mobilities versus the applied DC fields at superimposed AC fields $(1 \mathrm{kHz})$ of various strengths. As can be seen in the graph, at DC fields of $120 \mathrm{kV} / \mathrm{m}$ and above the mobilities are below the mobility when no AC field is superimposed. Error bars represent the standard deviation. 


\section{Chapter 4}

The differences in the overall mobilities of these data-groups (figure 4-5A), were not significant. Next to this possible indication for dielectrophoretic trapping, no other significant evidence was found. In B, it can be seen that the mobilities at DC fields of 40 $\mathrm{kV} / \mathrm{m}$ and below at almost all applied AC fields are higher than the mobilities observed at the absence of an AC field (dotted curve). Significant mobility increases for several data groups at applied DC fields of $8 \mathrm{kV} / \mathrm{m}$ and $20 \mathrm{kV} / \mathrm{m}$ are also found $(\mathrm{p}<0.05)$ at the addition of AC fields.

A

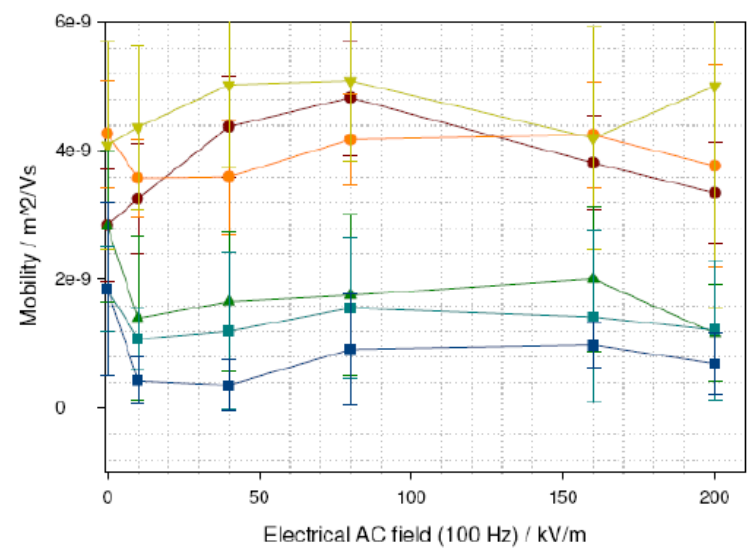

DC:

$$
\longrightarrow 10 \mathrm{kV} / \mathrm{m}
$$$$
\rightarrow 40 \mathrm{kV} / \mathrm{m}
$$

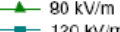$$
\rightarrow-160 \mathrm{kV} / \mathrm{m}
$$

B

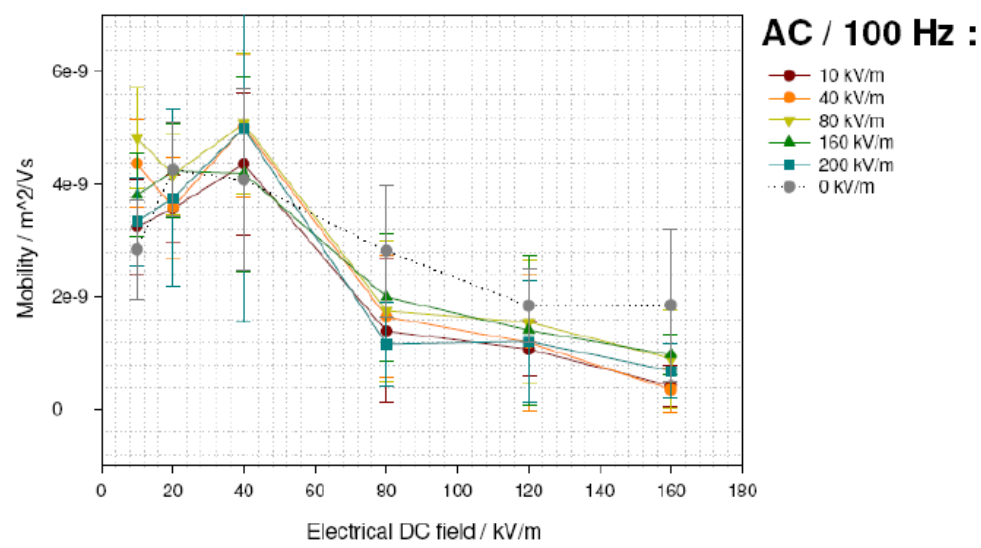

Figure 4-7. A, presents the go mobilities versus the applied AC fields $(100 \mathrm{~Hz})$ at DC fields of various strengths. As can be seen in the graph, at DC fields of $60 \mathrm{kV} / \mathrm{m}$ and above the mobilities are below the values when no AC field is superimposed. This is significant at DC fields of $80 \mathrm{kV} / \mathrm{m}$ with superimposed AC fields of 10 and $200 \mathrm{kV} / \mathrm{m}(\mathrm{p}<0.05)$ and for $\mathrm{DC}$ fields of $160 \mathrm{kV} / \mathrm{m}$ and superimposed AC fields of 10 and $40 \mathrm{kV} / \mathrm{m}(\mathrm{p}<0.05)$. The plot of the applied DC field with a strength of $10 \mathrm{kV} / \mathrm{m}$ is the same when comparing to figure 4-5 since the $\lambda$-DNA molecules moved fluent in these applied fields. In B, the mobility versus the applied DC field is presented. Error bars represent the standard deviation 
Several exceptions however were found. At a DC field of $20 \mathrm{kV} / \mathrm{m}$ and a superimposed AC field of $40 \mathrm{kV} / \mathrm{m}$, the mobility was significantly lower compared to the mobility without AC. In the same way the statistics show significantly lower mobilities at DC fields of 40 $\mathrm{kV} / \mathrm{m}(\mathrm{p}<0.05)$ at superimposed AC fields of 40 and $160 \mathrm{kV} / \mathrm{m}$ with respect to no AC. At the higher DC fields of 120 and $200 \mathrm{kV} / \mathrm{m}$, significant lower go mobilities are also found at the applied AC fields of 10,40 and $160 \mathrm{kV} / \mathrm{m}$ with respect to no $\mathrm{AC}(\mathrm{p}<0.05)$.

\subsubsection{Go-phase mobilities, $100 \mathrm{~Hz}$}

In figure 4-7A, the "go mobilities" of the $\lambda$-DNA molecules versus the applied AC fields $(100 \mathrm{~Hz})$ at DC fields of various strengths is presented. At DC fields below $40 \mathrm{kV} / \mathrm{m}$ where only fluent movement occurs, the data points are identical to the ones in figure 4-5 A and B. In figure 4-7 B, the dotted line represents the mobility of the $\lambda$-DNA molecules in the absence of an AC field. In A, it can be seen that above DC fields of $40 \mathrm{kV} / \mathrm{m}$ the mobilities seem to decrease to values below the $\mathrm{AC}$ field of $0 \mathrm{kV} / \mathrm{m}$. Indeed this difference is significant at DC fields of $80 \mathrm{kV} / \mathrm{m}$ at superimposed AC fields of 10 and $200 \mathrm{kV} / \mathrm{m}$ $(\mathrm{p}<0.05)$ and for DC fields of $160 \mathrm{kV} / \mathrm{m}$ at superimposed AC fields of 10 and $40 \mathrm{kV} / \mathrm{m}$ $(\mathrm{p}<0.05)$, representing a significant decrease in mobility when AC fields are applied. It should be realized that the trapping times of the molecules are not included, and increased trapping can not explain this observation. As was also concluded from the overall mobilities $(100 \mathrm{~Hz})$, further investigation at higher amplitudes is warranted since the provided results do not exclude a dielectrophoretic trapping mechanism.

\subsubsection{E Summary of the obtained mobilities $(100 \mathrm{~Hz}$ and $1 \mathrm{kHz})$}

In summary, a complex picture exists. The overall mobilities provided at both $100 \mathrm{~Hz}$ and $1 \mathrm{kHz}$ are mainly higher of value when compared to the mobilities provided without any AC field. In more detail, the overall mobilities at AC fields of $100 \mathrm{~Hz}$, and a DC field of 10 $\mathrm{kV} / \mathrm{m}$ show first a significant increase in mobility with increasing $\mathrm{AC}$ amplitude, followed by a decrease at applied AC fields of 160 and $200 \mathrm{kV} / \mathrm{m}(\mathrm{p}<0.05)$. A decrease is also found when AC fields of 0 and $10 \mathrm{kV} / \mathrm{m}$ are superimposed on the DC fields of 20 and $160 \mathrm{kV} / \mathrm{m}$, however at higher applied AC fields, the influence remains unclear. This could be pointing in the direction of dielectrophoretic trapping and needs to be further investigated. Turning now to the overall mobilities measured at $\mathrm{AC}$ fields of $1 \mathrm{kHz}$, these show a significant increase in the mobility for the DC fields of 8 and $20 \mathrm{kV} / \mathrm{m}(\mathrm{p}<0.05)$ at all added AC field strengths and for the DC field of $40 \mathrm{kV} / \mathrm{m}$ at an AC field strength of 0 and $40 \mathrm{kV} / \mathrm{m}$ $(\mathrm{p}=0.014)$. Only one significant decrease in mobility was found, namely at superimposed AC fields of 160 and $200 \mathrm{kV} / \mathrm{m}$ at the DC field of $40 \mathrm{kV} / \mathrm{m}(1 \mathrm{kHz})$. When investigating the "go" mobilities of these AC fields at the DC field of $40 \mathrm{kV} / \mathrm{m}(1 \mathrm{kHz})$, also a significant decrease in mobility is found. Next to this, we found that the "go" mobility at higher DC fields of $80 \mathrm{kV} / \mathrm{m}$ (all AC fields / $100 \mathrm{~Hz}$ ) and $120 \mathrm{kV} / \mathrm{m}$ (all AC fields / 100 $\mathrm{Hz}$ ), is below the mobilities provided without superimposed AC field. However, it remains 


\section{Chapter 4}

unclear whether this lower mobility is resulting from dielectrophoresis since the trapping times are excluded from these calculations.

\subsubsection{Pathways and trapping sites}

Next to the investigation of the mobilities, which was presented above, we also investigated the pathways and trapping sites of the molecules inside our nanoslits. By superimposing all of the video frames of a movie, we found preferential pathways and traps just as in the investigation of chapter $3^{1,2}$. An example of such an image is presented in figure 4-8A. In this figure a white dotted line is drawn, which is indicating a preferential pathway followed by the $\lambda$-DNA molecules. The large majority of DNA molecules was observed to follow this pathway, and it could be traced by connecting the small black dots visible in figure 4-8B (see black arrows).

\section{A}

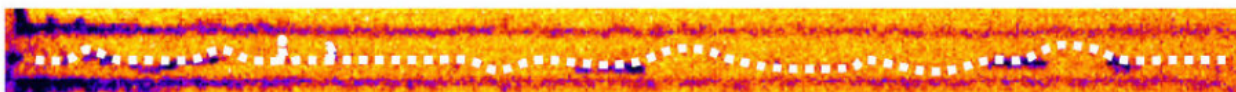

Pathway of DNA molecules

B

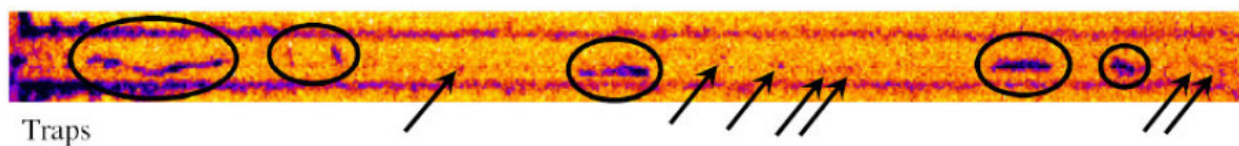

Figure 4-8. By superimposing all the frames of one movie, the pathways of the DNA molecules can be tracked inside a particular part of the nanoslit (above). The white dotted line represents such a pathway. In the picture below, this pathway is visible as small black dots (see arrows). Next to the pathways, traps are also found (under). The black circles are examples of sites were the $\lambda$-DNA molecules were temporarily delayed or trapped depending on the used conditions.

While studying the movements of the molecule, we could clearly see at the low DC fields (below $20 \mathrm{kV} / \mathrm{m}$ ) and at all AC fields $(10-200 \mathrm{kV} / \mathrm{m} ; 1 \mathrm{kHz})$ that the $\lambda$-DNA molecules followed their pathways through the nanoslit with a type of movement that can be described as a cycle of shrinking and stretching. This type of movement is typically found for large molecules in gel electrophoresis in the biased reptation regime (see also Chapter 2). In this regime, the DNA molecules move through the network of matrix pores which is also called the tube of the DNA molecule. Here, we also find pathways of molecules through the nanoslits and a similar type of movement, suggesting that the pathways might also represent the tubes of the DNA molecules, and that biased reptation occurs. We will further use the word caterpillar movement for this cycle of shrinking and stretching. An example of such movement is presented in figure 4-9, which shows three successive video frames. The applied DC field and AC field strength was $8 \mathrm{kV} / \mathrm{m}$ and $10 \mathrm{kV} / \mathrm{m}(1 \mathrm{kHz})$. The 


\section{AC/DC Field Dependent DNA Mobility in $20 \mathrm{~nm}$ High Nanoslits}

molecule is moving through the nanoslit from the left to the right. At $t=0 \mathrm{~s}$, the molecule has a small rounded shape, at $\mathrm{t}=0.16 \mathrm{~s}$ the molecule is stretched, which is followed again by shrinking in the length direction as can be clearly seen at $\mathrm{t}=0.37 \mathrm{~s}$. When only a DC field is applied, this caterpillar-like movement is also observed below $10 \mathrm{kV} / \mathrm{m}$. The trapping sites were also investigated in this study. In figure 4-8 B, positions of traps are indicated by black circles. At these locations, $\lambda$-DNA molecules were temporarily or permanently trapped just as was seen in the investigations of chapter $3^{1,2}$. In the DC measurements of chapter 3, a clear distinction could be made between fields where fluent movement and where intermittent movement was observed.

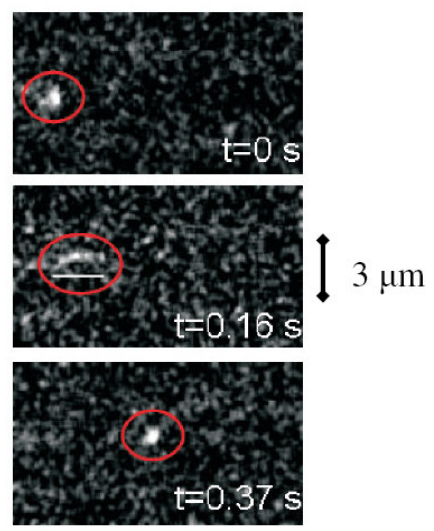

Figure 4-9. The DNA molecule is moving from the left till the right. In this figure we see three sequential frames in time. As can be seen, the DNA molecule moves in a sequence of stretching and shrinking. The applied DC field and AC field strength was $8 \mathrm{kV} / \mathrm{m}$ and $10 \mathrm{kV} / \mathrm{m}(1 \mathrm{kHz})$.

When AC fields are added, in all cases the trapping occurred at DC field strengths were this would also take place if AC field was applied. However, there was one interesting exception. At the applied DC field of $8 \mathrm{kV} / \mathrm{m}$ at all applied AC fields, the molecules were also trapped. The observed trapping times were however very short. When we compare these trapping times with those observed in DC fields of $40 \mathrm{kV} / \mathrm{m}$ and above at all superimposed $\mathrm{AC}$ fields, than we obtain here roughly 5\% of these trapping times. Remarkably the trapping times of the $\lambda$-DNA molecules in the DC fields of $8 \mathrm{kV} / \mathrm{m}$ became shorter with increasing AC field strength. Therefore this is not expected to be caused by dielectrophoretic trapping rather than by a mechanical trapping or other mechanism.

\subsubsection{DNA orientation, increasing mobilities and biased reptation}

As was presented in the previous paragraph and figure 4-8, we observed preferential pathways of the $\lambda$-DNA molecules. It can be seen in this figure that the pathways are not 


\section{Chapter 4}

always in the direction of the applied electric AC and DC fields. In the following part, we will consider the question how the orientation of a DNA molecule could lead to an increased mobility. A possible explanation for this might be found in the investigations of Ueda et al., Kaji et al. and Slater and Noolandi 9, 10,11. Both Ueda et al. and Kaji et al. showed that long DNA molecules $(48.5-2200 \mathrm{kbp})$ could be stretched in agarose or polyacrylamide gel when only AC fields were used at frequencies of $10 \mathrm{~Hz}{ }^{9,10}$. In AC fields with frequencies of $1 \mathrm{kHz}$, it was furthermore shown that the molecules became oriented (and not stretched) in the direction of the applied electric field ${ }^{9,10}$. When we assume that our $\lambda$-DNA molecules are also oriented in the direction of the applied field, then a possible explanation for the higher mobility of the $\lambda$-DNA molecules, can be found in the biased reptation model as described by Slater and Noolandi in $1985^{11}$. They described the biased reptation model for charged polymers in an environment of fixed obstacles and illustrated this with an example for DNA gel electrophoresis when DC electric fields were applied. The following part will now first briefly explain the biased reptation model (see also chapter 2). In brief, when the radius of gyration of the DNA is larger than the nominal pore size of the gel, the DNA must uncoil in order to enter the pore space. The DNA moves through the gel like a snake in a tube (the "reptation" part) and the electric force causes preferential motion in the direction of the electric field (the "biased" part) ${ }^{11,12,13,14 .}$ The DNA molecule is described as a chain of $\mathrm{N}$ vector segments $\mathrm{r}_{\mathrm{i}}$, with an end-to-end vector $h$ and is considered trapped in a tube (see also figure 4-11).

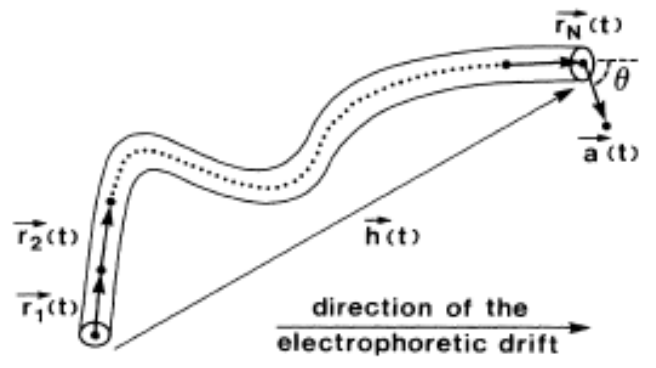

Figure 4-11. In the reptation theory, DNA molecules are considered trapped in a tube. The tube prevents lateral movement and is a result of the molecular environment of the molecule. The DNA molecule is modeled as a series of $\mathrm{N}$ vector segments $\mathrm{r}_{\mathrm{i}}$, with each a constant length $a$. With permission used from ref. 11 .

This tube is the result of the environment of the chain (as for example a gel matrix) and hinders any lateral movement of the molecule. Only one-dimensional movement along the tube axis is allowed. An electric field $\mathrm{E}$ applied in the $x$ direction, will lead to a center-ofmass electrophoretic mobility (see equation 1).

$$
\left\langle v_{c . m .}^{x}\right\rangle=Q E\left\langle h_{x}^{2}\right\rangle / L^{2} \xi_{c}
$$




\section{AC/DC Field Dependent DNA Mobility in $20 \mathrm{~nm}$ High Nanoslits}

In equation $1, v_{c . m}^{x}$ is the electrophoretic velocity of the center of mass of the chain, $\mathrm{Q}$ the total charge, E the applied electric field, $\left\langle h_{x}^{2}\right\rangle$ the average of the square of the end-to-end vector in the direction of the electric field, $\mathrm{L}$ the contour length of the chain and $\xi_{c}$ the curvilinear friction coefficient. For DNA molecules, $\mathrm{L}=\mathrm{N} d$, where $\mathrm{N}$ is the number of monomers (basepairs) and $d$ the length of one basepair. When the molecules are more oriented in the direction of the field in the higher superimposed AC fields, than the net force acting on the DNA molecule into the direction of the applied DC field will be larger, which could therefore explain the increase in mobility we find.

Thus in summary, if the DNA molecule is forced into a confined environment as for example a gel, it needs to stretch which will result in a larger end-to-end vector and thereby in a larger velocity. In the biased reptation model, the mobility will increase with increasing field strength as a result of this stretching. In our experiments, we could not measure a significant stretching of the molecule as a result of the high velocity of the DNA molecules and the error due to the used frame rate of the EMCCD camera. However, when we assume that the orientation of the molecules is increased with increasing AC field strength $(1 \mathrm{kHz})$ as was showed in the investigations of Ueda and Kaji, then the increased mobility we find could be explained by the biased reptation model of Slater and Noolandi ${ }^{11}$. When only focussing on this orientation, the influence of the net force of the applied electric field will increase when the molecule is more orientated with respect to the direction of the electric field. Furthermore, we see that the $\lambda$-DNA molecules stretch or shrink at fixed positions in the pathways. This suggests that these pathways can be regarded as the environmental tubes of the DNA molecules.

\subsection{Conclusion}

To investigate the occurrence of dielectrophoretic trapping inside our nanoslits, we superimposed AC fields $(10-200 \mathrm{kV} / \mathrm{m} ; 100 \mathrm{~Hz}$ and $1 \mathrm{kHz})$ on DC fields $(10-200$ $\mathrm{kV} / \mathrm{m}$ ). The electrokinetic transport behaviour of the $\lambda$-DNA molecules is investigated. As in our previous investigations of chapter 3 , we found fluent and intermittent movement of the $\lambda$-DNA molecules at different field strengths and preferential pathways. For each investigated DC fields below $20 \mathrm{kV} / \mathrm{m}$ and all superimposed AC fields $(1 \mathrm{kHz})$, a significant increase in mobility is found. This is not pointing into the presence of a dielectrophoretic trapping mechanism. This increase in mobility can be explained with the increase in the orientation of the molecule into the direction of the field with the biased reptation model ${ }^{11}$ and results of investigations in gel electrophoresis ${ }^{9,10}$. In the biased reptation model the orientation (and stretching) of the DNA molecules in the direction of the applied electric field results in a larger net force on the molecule and therefore an increasing mobility with increasing field strength. It was not possible to measure the lengths of the molecules, however when we assume an increase in orientation with increasing AC field strength $(1 \mathrm{kHz})$, as was showed in the investigation of Ueda et al. and 


\section{Chapter 4}

Kaji et al ${ }^{9,10}$, then the biased reptation model is in agreement with the provided results at the lower applied DC fields.

The other measured mobilities $(100 \mathrm{~Hz}$ and $1 \mathrm{kHz})$ provide a complex picture and next to a few exceptions, which need to be further investigated, no proof for the presence dielectrophoretic trapping mechanism could be found. When investigating the "go-phase" mobilities, some additional significant decreases in mobility are found for both frequencies. However, it remains unclear whether this lower mobility is resulting from dielectrophoresis since the trapping times were excluded from these "go-phase mobility" calculations.

\section{Acknowledgements}

Financial support by the Dutch Ministry of Economic Affairs through a NanoNed grant and Nano2Life is gratefully acknowledged.

\subsection{References.}

1. G. B. Salieb-Beugelaar, J. Teapal, J. van Nieuwkasteele, D. Wijnperlé, J. O. Tegenfeldt, F. Lisdat, A. van den Berg, J. C. T. Eijkel, Nano Lett., 2008, 8, 7, 17851790 .

2. G. B. Salieb-Beugelaar, J. Teapal, J. van Nieuwkasteele, D. Wijnperlé, J. O. Tegenfeldt, J. C. T. Eijkel, A. van den Berg, J. C. T. Eijkel, Proceedings of the microTAS Conference, 2007, 120 -1203.

3. K. R. Foster, F. A. Sauerm, H. Schwan, Biophys. J., 1992, 63:180-190.

4. R. Pethig, Y. Huang, X. B. Wang, J. Burt.. J. Phys. D: Appl. Phys., 1992, 25:881888.

5. C. L. Asbury, G. van den Engh, Biophys. J., 1998, 74, $1024-1030$.

6. C. F. Chou, J. O. Tegenfeldt, O. Bakajin, S. S. Chan, E. C. Cox, N. Darnton, T. Duke, R. H. Austin, Biophys. J., 2002, 83, $2170-2179$.

7. J. M. Chambers, W.S. Cleveland, B. Kleiner, P. A. Tukey, 1983. Graphical Methods for Data Analysis. Wadsworth \& Brooks / Cole

8. P. B. M. Roes, H. J. L. van Oorschot, 1978, Kansrekening en statistiek, Delftse uitgevers maatschappij, tweede druk.

9. M. Ueda, K. Yoshikawa, M. Doi, Polymer J., 1999, 31, 8, 637 - 644.

10. N. Kaji, M. Ueda, Y. Baba, Biophys. J., 2002, 82, 335 - 344.

11. G.W. Slater, J. Noolandi, 1985, Phys. Rev. Lett., 55, 151579 - 1582.

12. P. J. de Gennes, J. Chem. Phys., 1971, 55, 572 - 579.

13. L. S. Lerman, H. L. Frisch, Biopolymers, 1982, 21, 995 - 997.

14. O. J. Lumpkin, B. H. Zimm, Biopolymers, 1982, 21, 2315 - 2316. 
Filename: $\quad 4$ _ 2New Header_ACDC Field dependent DNA mobility_For_WPS_Final

Directory: $\quad$ C:LDocuments and SettingsiGeorgette.UTWENTEA174A94\Desktop

Template: C:LDocuments and SettingsiGeorgette.UTWENTEA174A94\Application DatalMicrosoft|Templates\Normal.dot

Title:

Subject:

Author:

Georgette

Keywords:

Comments:

Creation Date: $\quad$ 10/8/2009 11:24:00 AM

Change Number: 5

Last Saved On: $\quad$ 10/8/2009 11:51:00 AM

Last Saved By: Georgette

Total Editing Time: 21 Minutes

Last Printed On: $\quad$ 10/8/2009 11:51:00 AM

As of Last Complete Printing

Number of Pages: 16

Number of Words: $\quad 5,060$ (approx.)

Number of Characters: 28,848 (approx.) 


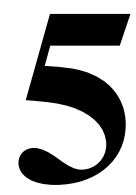

\section{DNA Mobility in 60 \& $120 \mathrm{~nm}$ High Nanoslits at Applied DC Fields}

In this chapter the mobility of the $\lambda$-DNA molecules in nanoslits of $60 \mathrm{~nm}$ and $120 \mathrm{~nm}$ height is investigated and the results are compared with the mobility found in the $20 \mathrm{~nm}$ high nanoslits. Interestingly, the mobility between $15-40 \mathrm{kV} / \mathrm{m}$ decreased in the order of $20 \mathrm{~nm}>60 \mathrm{~nm}>120 \mathrm{~nm}$. Preliminary molecular dynamic simulations confirmed the measured mobilities. We further investigated the presence of the "Stop \& Go" mobility, which we found in the $20 \mathrm{~nm}$ high nanoslits. Only in the $60 \mathrm{~nm}$ high nanoslits some "Stop $\&$ Go" mobility was found. Thus, the presence of the mechanical (and/or other) trapping mechanism is increasing with decreasing nanoslit height. Furthermore, the absence of preferential pathways in the $60 \mathrm{~nm}$ and $120 \mathrm{~nm}$ high nanoslits together with the roughly constant mobility of the $\lambda$-DNA molecules in all applied electrical DC fields, indicates that the molecules are not in the biased reptation regime as was suggested for the $20 \mathrm{~nm}$ high nanoslits.

Manuscript in preparation: G.B. Salieb-Beugelaar, J. Czerwinska, J.W. van Nieuwkasteele, D. Wijnperlé, A. van den Berg, J. C. T. Eijkel. 


\subsection{Introduction}

In the investigations shown in Chapter 3 we determined the mobility of $\lambda$-DNA molecules in $20 \mathrm{~nm}$ high nanoslits in applied electrical DC fields varying from $6-200 \mathrm{kV} / \mathrm{m}^{1,2}$. We found that the mobility of $\lambda$-DNA molecules (48502 basepairs (bp)), was field dependent. We also observed that the way in which the molecules were transported through the nanoslits depended on the field strength. When electrical DC fields below $30 \mathrm{kV} / \mathrm{m}$ were applied, the $\lambda$-DNA molecules were able to move fluently through the nanoslits. However, at electrical DC fields above $30 \mathrm{kV} / \mathrm{m}$ the $\lambda$-DNA molecules moved in an intermittent way. We described this as a "Stop and Go" movement. Apart from $\lambda$-DNA molecules, we also investigated the smaller Litmus DNA (2800 bp and digested with XbaI). The mobility of Litmus DNA was found to be lower than that of the $\lambda$-DNA molecules, which can open the door to new separation devices. Apart from the difference in mobility, the smaller Litmus DNA also moved in an intermittent manner in all applied electrical DC fields. In chapter 3 we suggested that this behavior could be explained by dielectrophoretic trapping and/or mechanical trapping of the DNA molecules inside the nanoslits.

The hypothesis of dielectrophoretic trapping was then first investigated (chapter 4). By the superimposition of electrical AC fields on applied DC fields, we increased the density of the electrical field lines inside the nanoslits. If a dielectrophoretic mechanism was at work, we would expect to find more $\lambda$-DNA molecules trapped inside the nanoslits with the addition of AC fields, however the larger part of the significant data showed an increase in mobility as a result of the applied AC field, especially at applied DC fields below $20 \mathrm{kV} / \mathrm{m}$. In a smaller part of the significant data we found a decrease in mobility.

Since the intermittent movement of the molecules was not explained by the data of chapter 4 , we decided to further investigate the behavior of $\lambda$-DNA molecules in nanoslits. In the study presented here, we used $60 \mathrm{~nm}$ and $120 \mathrm{~nm}$ high nanoslits and show that even in the $60 \mathrm{~nm}$ high nanoslits with less confinement the "Stop \& Go" behavior still occurs.

\subsection{Experimental protocol}

\subsubsection{The nanoslit devices}

Fused silica nanoslit devices with heights of $60 \mathrm{~nm}$ and $120 \mathrm{~nm}$ were manufactured as described in Appendix 1 at the end of this thesis. Of these devices both the surface roughness and the height of the nanoslits was studied. A typical AFM scan of a $120 \mathrm{~nm}$ high nanoslit is shown in figure 5-1. In figure 5-2 a typical AFM scan of the slit surface of this nanoslit is presented. The color code represents the difference in height. AFM tips were used with a radius of $7 \mathrm{~nm}$ and $2 \mathrm{~nm}$. The rms values for the nanoslit surfaces of both $60 \mathrm{~nm}$ and $120 \mathrm{~nm}$ slit height were ranging from $0.8 \mathrm{~nm}-2 \mathrm{~nm}$. We find larger nonuniformities on the surface than in the $20 \mathrm{~nm}$ slits, probably resulting from the longer etching step which was necessary to manufacture deeper nanoslits. The holes found in the $60 \mathrm{~nm}$ and $120 \mathrm{~nm}$ nanoslits are less deep when comparing to the $20 \mathrm{~nm}$ high nanoslits (see also figure 3-2). 
DNA Mobility in $60 \& 120 \mathrm{~nm}$ High Nanoslits at Applied DC Fields

Note that on the surface next to the nanoslit (figure 5-2) these inhomogenities are not present.

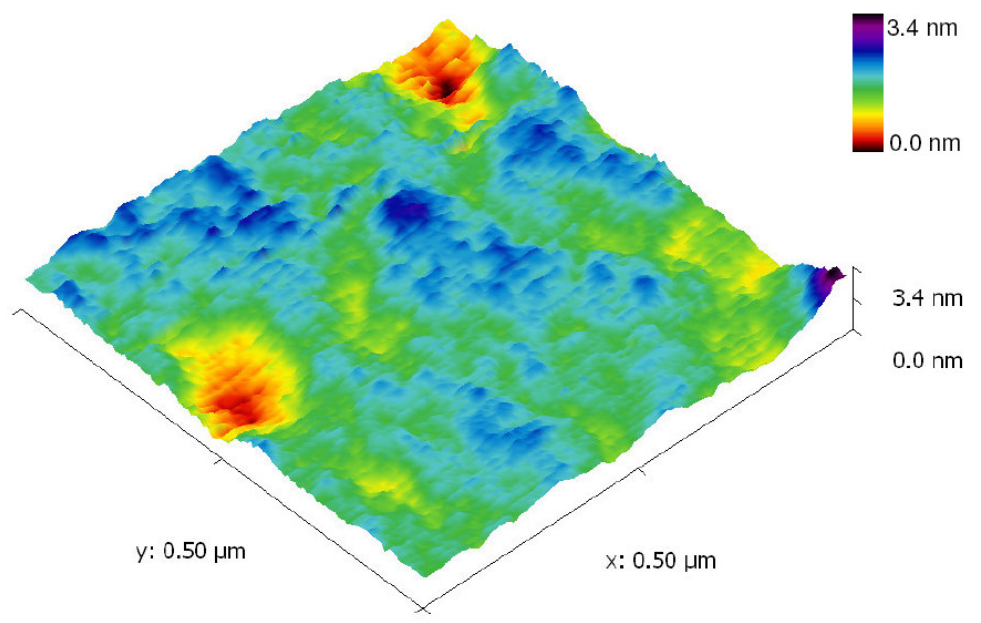

Figure 5-1 The surface roughness of a $120 \mathrm{~nm}$ nanoslit as measured by AFM using tips with a radius of $2 \mathrm{~nm}$. The rms values here are typically around $1 \mathrm{~nm}$, however we also found holes in the surface (as was seen in our previous investigations) and other non-uniformities on the surface.

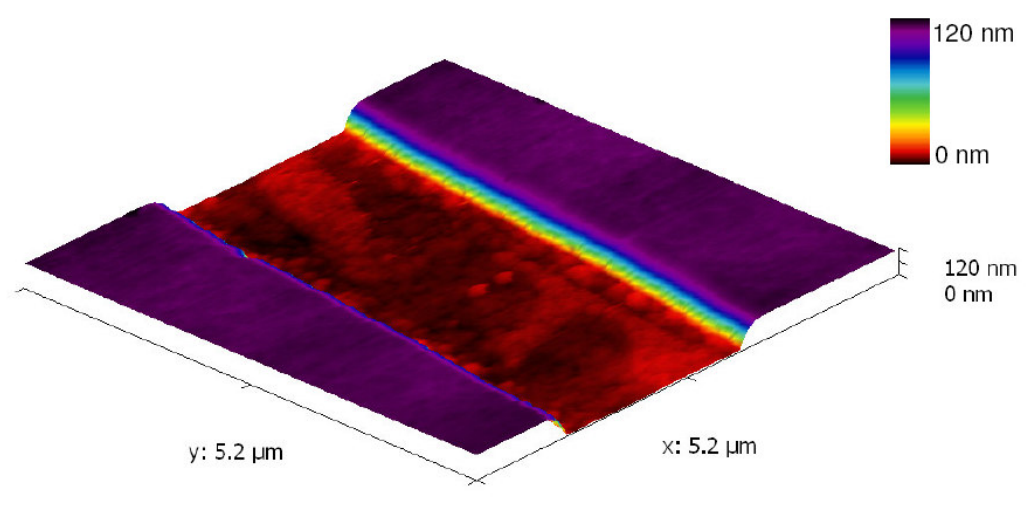

Figure 5-2 A cross-section of a $120 \mathrm{~nm}$ nanoslit as measured by AFM using a tip with a radius of 2 $\mathrm{nm}$. The rms roughness value of the larger nanoslit surface is typical $2 \mathrm{~nm}$. As can be clearly seen, all over the nanoslit surface inhomogenities are present. The differences in height of these inhomogeneities are up to $\sim 6 \mathrm{~nm}$. In figure 5-3 a typical example of these differences in height are given. 


\section{Chapter 5}
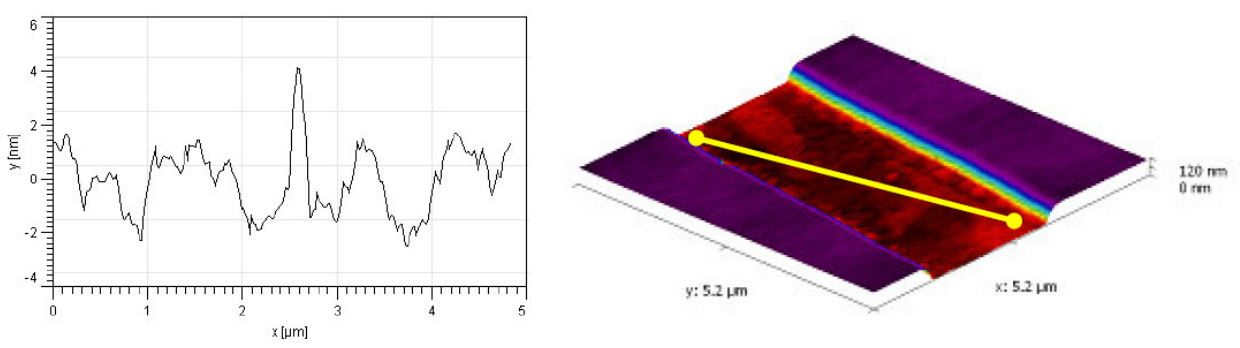

Figure 5-3. A typical section view of the surface roughness of a 120 high nanoslit surface (left). The section view represents the surface roughness along the yellow line drawn on the nanoslit surface of the AFM scan (right). The differences in height are up to $6 \mathrm{~nm}$. Height differences of 2-3 nm occur in patches of about $1 \mu \mathrm{m}$ width.

In figure 5-3 these larger inhomogeneities can be seen in the presented section view, which is taken from the nanoslit surface presented in figure 5-2. Height differences of $2-3 \mathrm{~nm}$ occur in patches of about $1 \mu \mathrm{m}$ width. The differences in height are up to $6 \mathrm{~nm}$. For the 60 $\mathrm{nm}$ high nanoslits, differences in height of up to $4.5 \mathrm{~nm}$ were found on the nanoslit surface.

\subsubsection{Electric setup}

The experimental setup was used as described in Appendix 1 at the end of this thesis. The Pt electrodes in the reservoirs were connected to a DC power supply (Keithley; high voltage source meter, model 2410). The YOYO-1 labeled $\lambda$-DNA/ Litmus molecules were pulled into the nanoslits by applying DC electrical fields between $10 \mathrm{kV} / \mathrm{m}$ and $2 \mathrm{MV} / \mathrm{m}$.

\subsubsection{Measurement procedure}

Acquisition and analysis of the video data was performed as described in Chapter 3. In brief, $\lambda$-DNA molecules were traced in successive video frames and from these data the velocities and mobilities were calculated. The experiments with the $60 \mathrm{~nm}$ high nanoslits were done in triplo, whereas the experiments with the $120 \mathrm{~nm}$ nanoslits were performed in duplo. For each experiment, all above mentioned field strengths were applied using different nanoslits. All measured data were pooled. The frame rate used was $22 \mathrm{~Hz}$. 


\subsection{Results and discussion}

\subsubsection{Mobility and nanoslit height}

DNA translocation experiments were carried out at different applied DC fields. In figure 54 an overview of the $\lambda$-DNA molecule mobility as found in the 60 and $120 \mathrm{~nm}$ high nanoslits is presented. As a comparison, the mobility measured in the $20 \mathrm{~nm}$ high nanoslits is included in the graph and is presented as the yellow line. Mobilities were determined up to a DC field strength of $100 \mathrm{kV} / \mathrm{m}$. Above this DC field strength we were not able to track most of the molecules due to excessive streaking of the DNA tracks, which was already manifested at lower field strengths in apparently elongated molecules. It is highly interesting to compare these results of the $60 \mathrm{~nm}$ and $120 \mathrm{~nm}$ with the mobilities found in the $20 \mathrm{~nm}$ high nanoslits. In figure 5-4 it can be clearly seen that the mobilities between the applied DC fields of $15-40 \mathrm{kV} / \mathrm{m}$ the mobility decreases in the order of $20 \mathrm{~nm}>60 \mathrm{~nm}>$ $120 \mathrm{~nm}$. Furthermore the mobility of the $\lambda$-DNA molecules remains roughly constant, for the $120 \mathrm{~nm}$ nanoslits $\sim 4.8 \cdot 10^{-9} \mathrm{~m}^{2} / \mathrm{Vs}$, and $6.1 \cdot 10^{-9} \mathrm{~m}^{2} / \mathrm{Vs}$ for the $60 \mathrm{~nm}$ high nanoslits in all applied electrical DC fields. The measured mobility in the $60 \mathrm{~nm}$ high nanoslits, is significantly $(p<0.001)$ higher when comparing to the mobility in the $120 \mathrm{~nm}$ high nanoslits in all presented DC fields. Thus, the lowest mobility of the $\lambda$-DNA molecules is found in the $120 \mathrm{~nm}$ nanoslits.

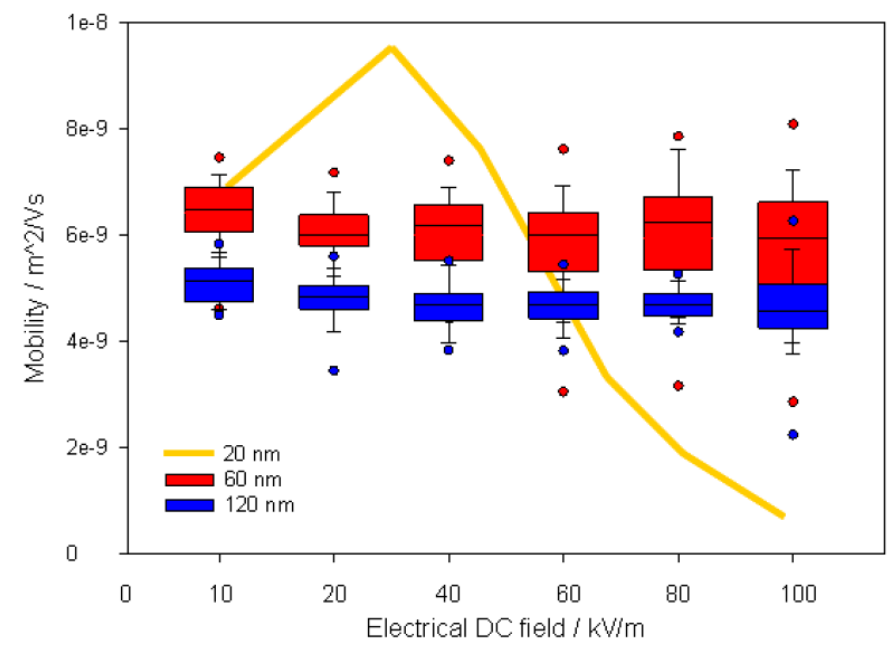

Figure 5-4. The $\lambda$-DNA mobility in 60 and $120 \mathrm{~nm}$ high nanoslits at different applied DC fields. The boxplots present the medians, the boxes indicates the $25^{\text {th }}$ to $75^{\text {th }}$ percentile of the population and the dots the $5^{\text {th }}$ and $95^{\text {th }}$ percentiles. It can be seen that the mobility in the 60 and $120 \mathrm{~nm}$ remains the same at all applied DC fields and that the mobility in the $120 \mathrm{~nm}$ high nanoslit is lower (significant difference $\mathrm{p}<0.001$ for all applied fields). The yellow line represent the mobilities of the fluent movements of the $\lambda$-DNA molecules in $20 \mathrm{~nm}$ nanoslits at DC field strengths of $10-100 \mathrm{kV} / \mathrm{m}$ (see also Chapter 3, figure 3-4) 
At present we have no explanation for this decrease in mobility with increasing nanoslit height. Possible influences of the degree of confinement on the $\lambda$-DNA conformation and hence on the mobility are being investigated by simulations. Interestingly, preliminary computer simulations done by J. Czerwinska, confirmed our measurements for electrical DC fields below $40 \mathrm{kV} / \mathrm{m}$ and all channel heights (data not shown). Next to this, when we compare the mobilities found in all our nanoslits with for example mobilities found for $\lambda$ DNA molecules in agarose gel electrophoresis ${ }^{3}$ ( $1 \%$ agarose gel), roughly $5.5 * 10^{-9} \mathrm{~m}^{2} / \mathrm{Vs}$ for $\lambda$-DNA at $1 \mathrm{kV} / \mathrm{m}$, we see that the mobilities in our nanoslits are in the same order of magnitude. Interestingly, for an agarose gel the pore size is typically between $100-300 \mathrm{~nm}^{3}$ and the geometry is different when comparing to a nanoslit. Thus, when regarding only the degree of confinement, we see in our nanoslits only one degree of nano-confinement, which is obviously not providing a larger mobility when comparing to the agarose gel (three dimensions of confinement).

\subsubsection{Pathways and trapping}

The transport behaviour of the $\lambda$-DNA molecules observed in these 60 and $120 \mathrm{~nm}$ high nanoslits is different from the behaviour in the $20 \mathrm{~nm}$ high nanoslits presented in the previous chapters. The first important difference is that no preferred pathways were found in these slits, as in the $20 \mathrm{~nm}$ high nanoslits. Furthermore, in the $20 \mathrm{~nm}$ slits, the $\lambda$-DNA molecules were transported through these pathways in a movement which can be described as a cycle of stretching and relaxing. Winkle et al. described this as "inchworm migration" ${ }^{4}$. In the 60 and $120 \mathrm{~nm}$ slits, apart from some exceptional stretching events at DC fields below $20 \mathrm{kV} / \mathrm{m}$ observed in both nanoslit heigths, the $\lambda$-DNA molecules moved in a fluent way at all DC fields below $60 \mathrm{kV} / \mathrm{m}$ and without showing preferred pathways. At electrical DC fields of $60 \mathrm{kV} / \mathrm{m}$ and above and only in the $60 \mathrm{~nm}$ high nanoslits, the $\lambda$-DNA molecules occasionally moved in an intermittent manner as was found previously for the $20 \mathrm{~nm}$ high nanoslits above DC fields of $30 \mathrm{kV} / \mathrm{m}$. However, there was a difference in behavior. Only a few molecules were transiently trapped inside the nanoslits, while many other $\lambda$-DNA molecules were able to pass without trapping. The molecules were roughly $10 \%$ of the total time trapped, which is in strong contrast with the $20 \mathrm{~nm}$ high nanoslits, where molecules were roughly $90 \%$ of the time trapped at electrical fields of $30 \mathrm{kV} / \mathrm{m}$ and above. At DC fields of $60 \mathrm{kV} / \mathrm{m}$, where the intermittent movement started in the $60 \mathrm{~nm}$ nanoslits, no intermittent movement was found in the $120 \mathrm{~nm}$ high nanoslits. Even when electrical fields up to $2 \mathrm{MV} / \mathrm{m}$ were applied, no stop and go movements were found in these slits apart from some exceptional events. This suggests that there exists a connection between the existence of pathways and the occurrence of intermittent movement at the higher DC fields. Since we were not able to find pathways in the 60 and $120 \mathrm{~nm}$ high nanoslits, and the mobility remained the same at all applied DC fields, we suggest that the $\lambda$-DNA molecules were not moving by biased reptation, as we hypothesized for the transport mode in the $20 \mathrm{~nm}$ high nanoslits. 
DNA Mobility in $60 \& 120 \mathrm{~nm}$ High Nanoslits at Applied DC Fields

\subsubsection{The force balance model}

The difference in mobility we found in our nanoslits can be compared with some very interesting results of Cross et al ${ }^{5}$. Interestingly, Cross et al. find lower mobilities in their $70 \mathrm{~nm}$ high nanoslits than in their $19 \mathrm{~nm}$ high nanoslits, a finding consistent with ours. In this work, DNA molecules of 2-10 kbp were used and nanoslits of 19 and $70 \mathrm{~nm}$ in height. Thus, the used DNA molecules were at least 5 times smaller when comparing to the $\lambda$ DNA molecules. The measured mobility for the $10 \mathrm{kbp}$ molecules in the $19 \mathrm{~nm}$ high nanoslits at an electrical DC field of $2.4 \mathrm{kV} / \mathrm{m}$ is $3.1 \cdot 10^{-8} \mathrm{~m}^{2} / \mathrm{Vs}$, roughly one order of magnitude larger than the mobility of the $\lambda$-DNA molecules at the lowest applied DC field $(6 \mathrm{kV} / \mathrm{m})$ in our $20 \mathrm{~nm}$ high nanoslits. This difference might be explained (next to the influence of the other experimental conditions) by the difference in length between the 10 $\mathrm{kbp}$ and the 5 times larger $\lambda$-DNA molecules, though it remains puzzling since we measured a lower mobility for the much shorter Litmus DNA. Cross et al. suggested that the surface interaction mainly determines the mobility. This surface interaction (friction) is a function of the relative size of the molecule compared to the dimensions of the nanoslit. Cross et al. supported this hypothesis by the length-dependent mobility for the 2-10 kbp DNA molecules in the $19 \mathrm{~nm}$ high nanoslits. With these observations we conclude that the length of the DNA, the degree of confinement, the nanoslit dimensions and the applied DC field strength are all of influence on the mobility of the DNA molecules. Cross et al ${ }^{5}$ presented a force-balance model for the mobility of DNA molecules (2-10 kbp) in $19 \mathrm{~nm}$ high nanoslits. In this investigation it was found that the mobility in the nanochannels scales with $\mathrm{N}^{-1 / 2}$ as a result of the friction of the DNA molecule with the buffer and the nanoslit surface. The model resulted in equation 1:

$$
\mu=\frac{\mu_{0}}{1+A N^{1 / 2}}
$$

Here $\mu$ is the measured mobility in the nanoslit, $\mu_{0}$ the mobility in free solution, $N$ is the number of Kuhn segments and $\mathrm{A}$ is a constant. $\mu_{0}$ and $\mathrm{A}$ are allowed to vary for fitting purposes. The free solution mobility in this investigation was established as $\sim 1.06^{*} 10^{-8}$ $\mathrm{m}^{2} / \mathrm{Vs}$ and the parameter $\mathrm{A}$ is $2.4 * 10^{-7} \mathrm{~m}^{2} / \mathrm{Vs}$.

In our investigation, the $\lambda$-DNA molecules used are $48 \mathrm{kbp}$. When we apply this model to our mobility data for the $20 \mathrm{~nm}$ nanoslits, this model would not fit since we find a fielddependent mobility in these nanoslits. If we use this model for the 60 and $120 \mathrm{~nm}$ nanoslits, then the value of the parameter A used by Cross does not result in a good fit either. We will therefore consider Cross' model in more detail. As mentioned, Cross et al. presented a force-balance model of DNA interacting with the buffer and the channel surface. On each DNA molecule the electrical force $\mathrm{F}_{\mathrm{e}}=\mathrm{qNE}$ is transporting the molecules through the nanoslits. Here $\mathrm{q}$ is the electrical charge per monomer and $\mathrm{N}$ is the number of monomers. The DNA molecules are slowed down by the friction with the buffer $F_{b}=f_{b} v N$ and the surface friction $F_{s}=f_{s} v N \alpha$. Here $f_{b}$ is the buffer friction per base pair, $f_{s}$ the surface friction per base pair, $\mathrm{v}$ the velocity of the molecule, $\mathrm{N}$ the number of monomers and $\alpha$ is the 


\section{Chapter 5}

fraction of the molecule interacting with the surface. When the electrical force $F_{e}$ is balanced with the buffer friction $F_{b}$ and the surface friction $F_{s}$, we get (eq. 2):

$$
\mu=\mu_{0}\left(1+\frac{f_{s}}{f_{b}} \alpha\right)^{-1}
$$

Here, $\mu$ is defined by $v=\mu \mathrm{E}$ and $\mu_{0}$ is the free solution mobility which equals the ratio $q / f_{b}$. Cross et al. assumed that the buffer and friction forces per base pair are not depending on length, therefore the length dependence is entirely within $\alpha$. Before we continue, we now must first describe the confinement of the $\lambda$-DNA molecules in our nanoslits. Since our DNA molecules are longer, we will not use the pancake-like confinement as developed by Brochard et al. and used by Cross et al. in his model. First, when $\lambda$-DNA molecules are in a buffer solution, the radius of gyration $\mathrm{R}_{\mathrm{g}}$ (see also chapter 2$)^{6}$ is described by equation $3^{7}$ :

$$
R_{g}=\left(P_{d y e} w_{e f f} L_{d y e}^{3}\right)^{1 / 5}
$$

Here, $P_{\text {dye }}$ is the persistence length of the $\lambda$-DNA molecule after the intercalation with YOYO- $1, w_{\text {eff }}$ is the effective width of the molecule and $\mathrm{L}_{\text {dye }}$ is the contour length of the $\lambda$ DNA molecule after YOYO-1 is intercalated ${ }^{7-11}$. In our experiments $\mathrm{P}_{\text {dye }} \approx 67 \mathrm{~nm}, \mathrm{~L}_{\text {dye }} \approx$ $20.5 \mu \mathrm{m}$ and $\mathrm{w}_{\text {eff }} \approx 4 \mathrm{~nm}$, which is resulting in $\mathrm{R}_{\mathrm{g}} \approx 1098 \mathrm{~nm}$. Since this $\mathrm{R}_{\mathrm{g}}$ is larger than the height of the nanoslits, the $\lambda$-DNA molecule will extend when it is pulled in the nanoslits by the applied DC field. Since the height of the nanoslits is smaller than the Kuhn length or of the order of the Kuhn length (see also Chapter 2$)^{6}$, we can use the blob theory to calculate an average extended radius $\mathrm{R}_{\mathrm{e}}$ using equation 4 given by Odijk ${ }^{12}$

$$
R_{e} \approx\left(L_{d y e} P_{d y e}\right)^{1 / 2}\left(\frac{L_{d y e} w_{e f f}}{P_{d y e} h}\right)^{1 / 4}
$$

Here, $\mathrm{h}$ is the height of the nanoslit. When using this equation, we find an average extended radius for the 60 and $120 \mathrm{~nm}$ high nanoslits of $2.49 \mu \mathrm{m}$ and $2.09 \mu \mathrm{m}$ respectively. The number of contacts $\mathrm{C}$ between surface and DNA for our $\lambda$-DNA molecules following Cross et al. is given by

$$
C=\Theta \pi R_{e}^{2}
$$

Here $\Theta$ is the number of contact points per unit area. The fraction of the DNA molecule interacting with the surface is

$$
\alpha=C / N
$$


DNA Mobility in $60 \& 120 \mathrm{~nm}$ High Nanoslits at Applied DC Fields

If we replace $\left(f_{s} / f_{b} * \alpha\right)=b$ in equation 2 , rewriting will subsequently result in equation 7 .

$$
\mu=\mu_{0}\left(1+\frac{f_{s}}{f_{b}} \alpha\right)^{-1}=\mu_{0}(1+b)^{-1}
$$

This equation allows comparison with the measurement data. Calculation of $\mathrm{b}$ on the basis of our measured mobility values for the 60 and $120 \mathrm{~nm}$ nanoslits results in $b=-0.38$ and $b$ $=-0.21$ respectively. Clearly the friction with the surface or $\alpha$ cannot be $<0$, and therefore this model does not fit for our data and the surface friction model of Cross et al. does not offer an explanation for the observed mobility values. In fact any friction-based model will struggle to explain a mobility that increases with increasing confinement. It is therefore strongly recommended to further investigate this regime, as is presently being done in collaboration with J. Czerwinska by using molecular simulation models. As was seen in the investigation of Cross, when the DNA molecules indeed have a mobility which is related to their confinement and length (as we see for the $10 \mathrm{kbp}$ molecules in the $70 \mathrm{~nm}$ high nanoslits) and which is independent of the applied electrical field (the $\lambda$-DNA molecules in the 60 and the $120 \mathrm{~nm}$ high nanoslits), than this could open the door to separation applications, just like the field-dependent mobility described in chapter 3 . It could be possible that this behaviour starts above a certain length, since Cross et al. were not able to separate the 2-10 kbp DNA molecules from each other in the $70 \mathrm{~nm}$ high nanoslits.

\subsubsection{Behavior at fields above $1 \mathrm{MV} / \mathrm{m}$}

We also investigated the DNA transport behavior at higher DC fields. When electrical fields of $1.4 \mathrm{MV} / \mathrm{m}$ and $1.6 \mathrm{MV} / \mathrm{m}$ were applied for the 60 and $120 \mathrm{~nm}$ high nanoslits respectively, DNA plugs formed at the entrances of the nanoslits. This plug formation could possibly be the result of the high density of the electrical field lines at the entrances of the nanoslits, leading to dielectrophoresis ${ }^{13}$. At a certain moment in time, when the plug was large it was released with a high velocity into the nanoslit. An example of such a plug is presented in figure 5-3. Not all plugs had the same size and shape. We even found plugs inside the nanochannels (in roughly 1 nanoslit out of 10). We found that when we decreased the field strength, the plug dissolved slowly and the $\lambda$-DNA molecules moved almost separately through the nanoslit. No proof for huge DNA clots was found. This behavior could be very interesting for pooling a particular amount of DNA molecules at entrances of nanoslit devices and subsequently injecting it by a change of field strength, starting the transport of the DNA molecules at once. 
Chapter 5

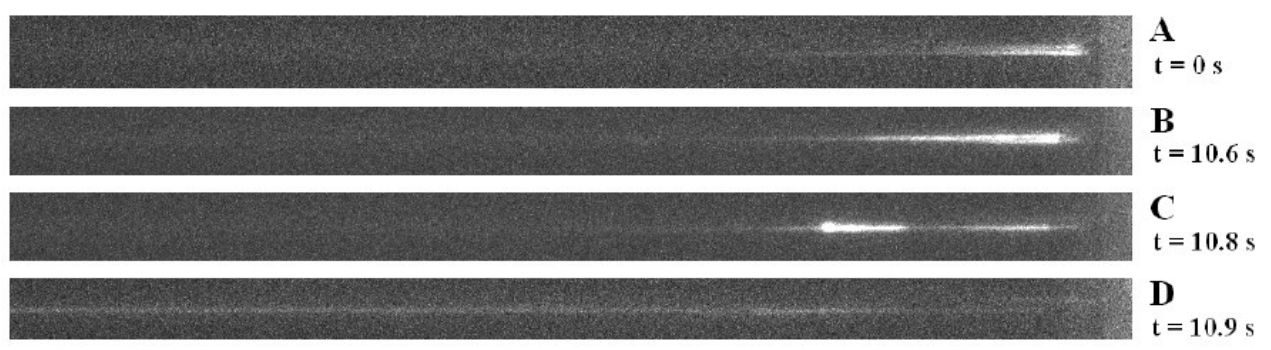

Figure 5-3. At fields of $1.4 \mathrm{MV} / \mathrm{m}$ and $1.6 \mathrm{MV} / \mathrm{m}$ and higher for the $60 \mathrm{~nm}$ and $120 \mathrm{~nm}$ high nanoslits respectively, plugs were formed at the entrances of the nanoslits (right side). The plug formation here is inside a $120 \mathrm{~nm}$ nanoslit. $\lambda$-DNA molecules are accumulating inside the nanoslit, next to the entrance. At a certain moment in time, the plug was released into the nanoslit (from right to the left).

\subsubsection{Preliminary results with Litmus DNA}

Apart from the $\lambda$-DNA molecules, we were also interested in the transport behavior of XbaI digested Litmus DNA molecules (see also Chapter 3$)^{2}$. When applying DC field strengths varying between $0 \mathrm{~V} / \mathrm{m}$ and $2 \mathrm{MV} / \mathrm{m}$, we were unfortunately not able to see movement of the Litmus DNA molecules inside both $60 \mathrm{~nm}$ and $120 \mathrm{~nm}$ nanoslits. This is probably due to the insufficient quality of the microscope and camera. While investigating the high field behavior in the $60 \mathrm{~nm}$ high nanoslits, we observed that the Litmus DNA molecules accumulated inside the nanoslits at certain locations which were visible as dots, and also at the channel entrance.

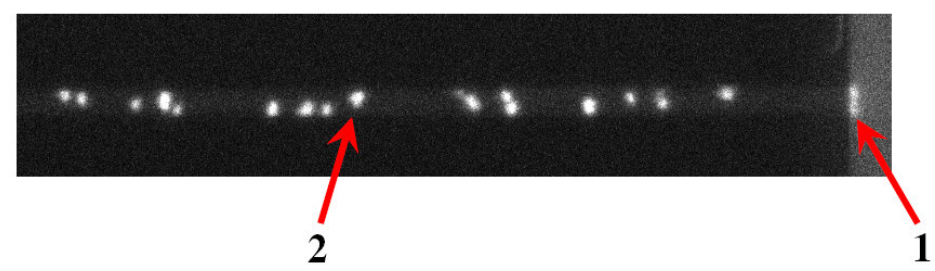

Figure 5-6. At an applied electrical field of $2 \mathrm{MV} / \mathrm{m}$, we see Litmus DNA molecule accumulations as dots inside the nanoslits and at the entrance of the nanoslit. Unfortunately, we were not able to follow the movements of the Litmus molecules through the nanoslits.

An example of this is presented in figure 5-6. After reversing the electrical field (- 2 $\mathrm{MV} / \mathrm{m}$ ), most of the dots disappeared rapidly. This is a very interesting result, since it contradicts the possible explanation that the accumulation is due to dielectrophoresis. If the accumulation was caused by dielectrophoresis, then the molecules would not disappear on field reversion. 


\subsection{Conclusion}

In this chapter, we investigated whether the intermittent ("stop and go") movement of the $\lambda$-DNA molecules in $20 \mathrm{~nm}$ nanoslits as presented in Chapter 3 and 4 also occurred in larger height nanoslits. Here, nanoslits with a height of 60 and $120 \mathrm{~nm}$ were used and DC fields were applied. Only in the $60 \mathrm{~nm}$ high nanoslits, intermittent movement was seen, but only of a small percentage of the DNA molecules whereas most molecules moved fluently through the nanoslit. The mobility of $\lambda$-DNA was furthermore independent of the field strength and no preferential pathways were observed. In all aspects the DNA therefore seemed to be in a different movement regime than in the biased reptation regime which was found in the $20 \mathrm{~nm}$ slits. Interestingly, the mobility between $15-40 \mathrm{kV} / \mathrm{m}$ decreased in the order $20 \mathrm{~nm}>60 \mathrm{~nm}>120 \mathrm{~nm}$.

The smaller Litmus DNA (XbaI digested) was also investigated. We were however not able to track these molecules inside the 60 and $120 \mathrm{~nm}$ high nanoslits. Surprisingly, we found at very high fields of $2 \mathrm{MV} / \mathrm{m}$ indications that the Litmus molecules were trapped at certain locations inside the nanoslits.

\section{Acknowledgements}

We thank Joost Swennenhuis for the help with the agarose gel electrophoresis of the XbaI digested Litmus DNA. Justyna Czerwinska is thanked for the fruitful collaboration. Financial support by the Dutch Ministry of Economic Affairs through a NanoNed grant and Nano2Life is gratefully acknowledged.

\subsection{References}

1. G. B. Salieb-Beugelaar, J. Teapal, J. van Nieuwkasteele, D. Wijnperlé, J. O. Tegenfeldt, F. Lisdat, A. van den Berg, J. C. T. Eijkel, Nano Lett., 2008, 8, 7, 17851790.

2. G. B. Salieb-Beugelaar, J. Teapal, J. van Nieuwkasteele, D. Wijnperlé, J. O. Tegenfeldt, A. van den Berg, J. C. T. Eijkel, Proceedings of the MicroTas Conference 2007, 1201-1203.

3. C. Heller, T. A. J. Duke, J. L. Viovy, Biopolymers, 1994, 34, 249 - 259.

4. D. H. van Winkle, A. Beheshti, R. L. Rill, Electrophoresis, 2002, 23, 15-19.

5. J. D. Cross, E. A. Strychalski, H. G. Craighead,J. App. Phys., 2007, 102, 024701.

6. G. B. Salieb-Beugelaar, K. D. Dorfman, A. van den Berg, J. C. T. Eijkel, Lab-on-aChip, 2009, 9, 2508 - 2523.

7. D. W Schaefer, J. F. Joanny, P. Pincus, Macromolecules, 1980, 13, 1280.

8. P. K. Lin, K. Lin, C. C. Fu, K. C. Lee, P. K. Wei, W. W. Pai, P. H. Tsao, Y. L. Chen, W. S. Fann, Macromolecules, 2009, 42 (5), 1770-1774.

9. W. Reisner, J. P. Beech, N. B. Larsen, H. Flyvbjerg, A. Kristensen, J. O. Tegenfeldt, Phys. Rev. Let., 2007, 99, 058302. 


\section{Chapter 5}

10. J. T. Mannion, C. H. Reccius, J. D. Cross, H. G. Craighead, Biophys. J., 2006, 90, $4538-4545$.

11. L. H. Thamdrup, A. Klukowska, A. Kristensen, Nanotechnology, 2008, 125301

12. T. Odijk, Phys. Rev. E, 2008, 77, 060901.

13. L. Ying, S. S. White, A. Bruckbauer, L. Meadows, Y. E. Korchev, D. Klenerman, Biophys. J., 2004, 86, 1018-1027. 
Filename: 5_New Header_60 nm \& $120 \mathrm{~nm}$ DC_For_WPS_Final Directory: $\quad$ C:LDocuments and Settings/Georgette.UTWENTEA174A94\Desktop

Template:

C:LDocuments and SettingsiGeorgette.UTWENTEA174A94\Application DatalMicrosoft|Templates $\backslash$ Normal.dot

Title: 1

Subject:

Author:

Keywords:

Comments:

Creation Date: $\quad$ 10/8/2009 10:51:00 AM

Change Number: 2

Last Saved On: $\quad$ 10/8/2009 10:51:00 AM

Last Saved By: Georgette

Total Editing Time: 1 Minute

Last Printed On: $\quad$ 10/8/2009 10:57:00 AM

As of Last Complete Printing

Number of Pages: 12

Number of Words: $\quad 3,686$ (approx.)

Number of Characters: 21,016 (approx.) 


\section{6}

\section{The Charge-Patterned Nanoslit}

In this chapter we present the manufacturing of a newly designed nanoslit device. Inside the nanoslit a patterned surface consisting of $\mathrm{SiO}_{2}$ and $\mathrm{Al}_{2} \mathrm{O}_{3}$ was manufactured which under the proper circumstances will lead to a patterned surface charge. By aligning this patterned surface with a slope in the length direction of the nanoslit, it is expected that a separation of biomolecules can be obtained when the height of the nanoslit is small enough to allow for intensive Coulomb interaction between the biomolecules and the surface. The dimensions of these patterns were chosen according to the limitations of the photolithography and manufactured in a Borofloat wafer. In order to obtain a smooth surface of the patterns we applied chemical mechanical polishing. In another Borofloat wafer the microchannels and nanoslit were manufactured. After anodic bonding the wafers were diced and we were in a preliminary experiment able to successfully fill the nanoslit with $0.1 \mathrm{mM} \mathrm{NaAc}$ buffer $(\mathrm{pH}=5)$. 


\subsection{Introduction}

In addition to the nanoslit devices used in the previous chapters of this thesis, a nanofluidic device was developed, possessing a charge-patterned surface to separate biomolecules in a continuous flow without the use of an applied electrical field. We decided to manufacture a nanoslit with a patterned surface of $\mathrm{SiO}_{2}$ and $\mathrm{Al}_{2} \mathrm{O}_{3}$ (see figures 6.1 and 6.2). This patterned surface will only be present on one of the sides of the nanoslit part of the device. The surface charge of the pattern can be adjusted by the ionic strength and $\mathrm{pH}$.

The aim of the structure is to separate biomolecules in a continuous flow. In the projected future use of the device, the composition of the used buffer will be extremely important. For example, at $\mathrm{pH}=5$ the $\mathrm{SiO}_{2}$ is negatively charged and $\mathrm{Al}_{2} \mathrm{O}_{3}$ is positively charged. Furthermore, when the concentration of the buffer is $0.1 \mathrm{mM}$, the double layer thickness is roughly $30 \mathrm{~nm}$. Thus at a $\mathrm{pH}=5$, a landscape of oppositely charged double layers are generated on the patterned surface, extending into the solution. In a $100 \mathrm{~nm}$ high nanoslit at this buffer concentration, the double layers are not overlapping yet. The flow of a charged (bio) molecule with a length longer than $40 \mathrm{~nm}$ however is expected to be influenced by the presence of these double layers on one side of the nanoslit. Also, biomolecules such as proteins or DNA are complex macromolecules with conformations that are dependent on both their composition as their environment (for example $\mathrm{pH}$ or salt concentration and buffer composition). Apart from use in their native or nano-confined conformations, biomolecules such as proteins can be straightened and charged by treating them with a detergent. According to the detergent chosen, the final protein surface charge will be positive or negative. This charged patterned nanoslit device is expected to give new possibilities to separate biomolecules by adjusting the double layer thickness and zeta potentials, to perform a continuous flow separation on both charge and length. In this chapter we will present the device layout, material choice and the manufacturing steps of this device.

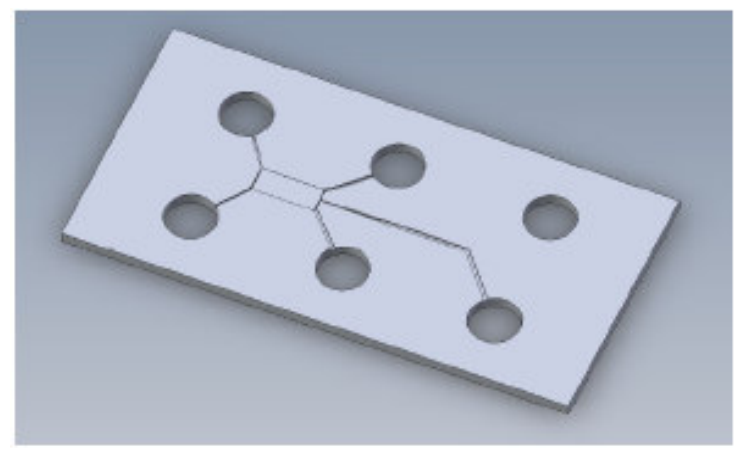

Figure 6-1. A picture of the device. For more details see also figure 6-2. This device is composed of two separately manufactured Borofloat wafers. In one wafer the nanoslit and the microchannel is etched, whereas in the other wafer a pattern of borofloat and aluminumoxide is manufactured. This pattern is manufactured in such a way that it will be located inside the nanoslit part of the device. 


\section{The Charge-Patterned Nanoslit}

\subsection{Device layout}

The device is manufactured by bonding two separately processed Borofloat wafers, a top and a bottom wafer. In one wafer the nanoslit and the microchannels are manufactured, whereas in the other wafer a pattern of Borofloat and aluminumoxide is manufactured. In figure 6-1, a picture of the device is presented. The dimensions of the lines are chosen according to the limits of the used manufacturing processes. An overview of the exact dimensions of each designed device is presented in an appendix at the end of this chapter. Fabrication details are described in the next section of this chapter. Figure 6-2 schematically presents the layout of the device, which possesses two buffer inlets and a sample inlet. In continuous flow, buffer will be pumped into the patterned nanoslit area through the microchannels. At the same time, sample can be pumped into the device through the sample inlet (figure 6-2A). Both injection microchannels as the sample microchannel have a depth of $15 \mu \mathrm{m}$ to reduce the fluidic resistance. The fluid flow through the nanoslit drains away through the microchannels at the other side of the nanoslit towards the two outlets.

A

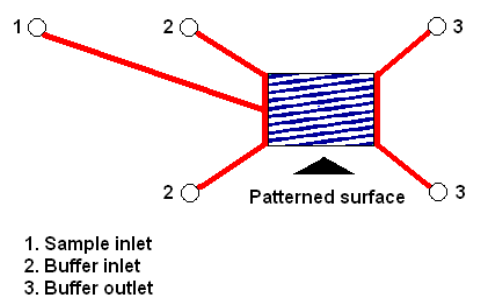

B

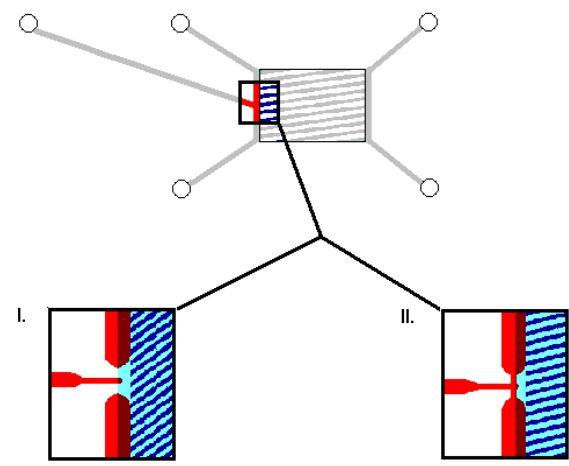

Figure 6-2. The device is schematically presented in A. Microchannels are indicated in red and nanochannels in light blue. The patterned lines composed of $\mathrm{Al}_{2} \mathrm{O}_{3}$ are dark blue. Two buffer inlet microchannels are directly connected to the patterned nanoslit area (inlet number 2). Also a sample inlet microchannel connects to the nanoslit (inlet number 1). The fluid flow through the nanoslit is drained away through the microchannels at the opposite side of the nanoslit (outlet number 3 ). In B, two alternative injection layouts are presented. In I. the sample is injected into the nanoslit area (lightblue color) just a few $\mu \mathrm{m}$ before the patterned area without direct connection with the buffer microchannels. In II. the sample is injected into the nanoslit, while connected with the buffer microchannels.

Two alternative injection layouts were designed, which are presented in figure 6-2B. In design B-I, the buffer and sample microchannels are not connected. Here the sample is pumped directly in the nanoslit area (light blue color in figure 6-2 B) however a few $\mu \mathrm{m}$ 
before the pattern and without direct connection to the buffer microchannels. In design BII., the sample microchannel is connected with the buffer microchannels.

\subsection{Choice of the material}

There are stringent requirements to the properties of the materials used in this device. First, the device should be transparent (at least at one side) to enable the detection of single fluorescent DNA molecules by optical fluorescence microscopy. Second, the material should be inert, not to contaminate the sample solution. We first considered fused silica since it has the advantage of being pure silicon dioxide, preventing possible leaching out of salts. A drawback however is the extremely high annealing temperature of $1100{ }^{\circ} \mathrm{C}$, which is necessary to get a good bond between both the wafer containing the microchannels and the nanoslit and the wafer which contains the pattern. This high temperatures might ruin the pattern, since it will be manufactured of two different materials and thus will have two different expansion coefficients. Our first choice was therefore glass, e.g. Borofloat 33 ${ }^{\mathrm{TM}}$, which has an annealing temperature of $650{ }^{\circ} \mathrm{C}$. Prior to the manufacturing of the device, the Borofloat was tested on the possibility of manufacturing a pattern onto it which can stand the annealing temperature of $650{ }^{\circ} \mathrm{C}$. The results of this test were positive.

\subsection{Manufacturing process}

As mentioned before, the device consists of two bonded wafers. For the entire manufacturing process, four different masks were used which will be mentioned in the next sections. First the process for the top wafer is described (containing the microchannels and nanoslit), then the process for the bottom wafer (containing the pattern) and finally the bonding and dicing procedure.

\subsubsection{The top wafer}

In figure 6.3 the fabrication process for the top wafer is presented. The top wafer is a 100 $\mathrm{mm}$ Borofloat $33^{\mathrm{TM}}$ wafer from Schott. Prior to the first lithographic step it was cleaned by rinsing it for 10 seconds in a $25 \%$ (W/V) $\mathrm{KOH}$ solution at $75{ }^{\circ} \mathrm{C}$ followed by an ultrasonic bath treatment with deionized (DI) water for 10 minutes. Next, the wafer was quickly rinsed in DI water, immersed in fuming nitric acid for 10 minutes and as a final step it was again rinsed in DI water. After a dehydration step $\left(10\right.$ minutes at $\left.95^{\circ} \mathrm{C}\right)$ an HexaMethylDiSilazan (HDMS) layer as adhesion layer is applied on the surface of the wafer by a the use of a vapor phase treatment $\left(10\right.$ minutes at $\left.150{ }^{\circ} \mathrm{C}\right)$, which is necessary in order to prevent undercutting during the etching steps. Subsequently a positive photoresist Fujifilm OiR 907/17 is spin coated (20 seconds at $4000 \mathrm{rpm}$ ). The photoresist is dried for 1 minute at $95{ }^{\circ} \mathrm{C}$ and exposed for 4.2 seconds at $12 \mathrm{mWatt} / \mathrm{cm}^{2}$ through the "nanoslit" mask by ElectroVison mask aligner in vacuum-hard contact mode at 1.5 bar. 


\section{The Charge-Patterned Nanoslit}

Then, after a post exposure bake $\left(1\right.$ minute at $\left.95^{\circ} \mathrm{C}\right)$ the photoresist is developed in Fujifilm OPD 4262 developer for 40 seconds and baked for 20 minutes at $110{ }^{\circ} \mathrm{C}$. To remove any residues from the photolithography process and start with a plane etching process, a descum step is introduced for 5 minutes in a UV-ozone reactor (UVP inc. PR-100) just before wet etching.

1.

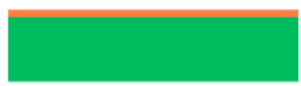

Vapour phase HMDS photo resist OiR 907/17

2.

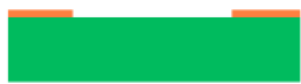

Develop photo resist OiR 907/17

3.

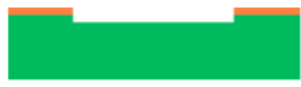

Wet etch nanoslit (BHF/HCl, 9:1)

4.

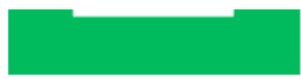

Resist strip in $100 \% \mathrm{HNO}_{3}$

Figure 6-3. The fabrication process for creating the nanoslit into the topwafer.

First, the nanoslit is etched in a freshly prepared mixture of buffered $\mathrm{HF}$ and $\mathrm{HCl}$, mixed in a ratio 9:1. The $\mathrm{HCl}$ is added to get a smoother etch result. After preparation, the mixture is allowed to cool down to $18{ }^{\circ} \mathrm{C}$ for about 30 minutes. For a $100 \mathrm{~nm}$ nanoslit the etch time was 3 minutes and 20 seconds. To determine the depth of the nanoslit and the surface roughness of the bottom of the slit an AFM scan was performed. The result of a typical AFM scan is shown in figure 6.4 .

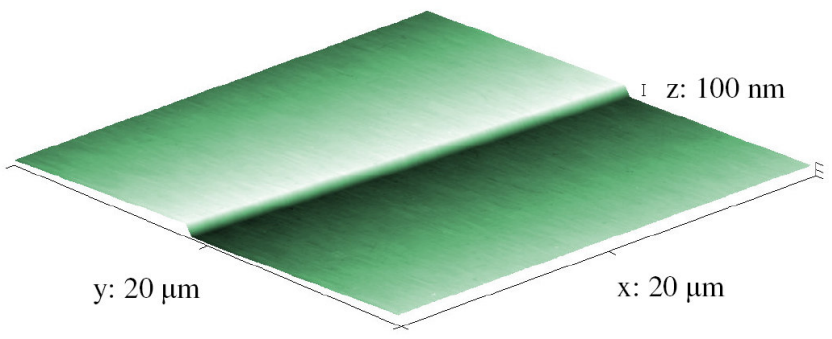

Figure 6-4. The surface roughness and the depth of a nanoslit as measured by AFM using tips with a radius of $2 \mathrm{~nm}$. The 3D plot of the scan is stretched in $\mathrm{z}$ direction and thus not on scale with the $\mathrm{x}$ and the $y$ axis. 
Of the nanoslit area the surface roughness was also determined. A typical AFM scan is shown in figure 6-5. The rms values found for the surface roughness are usually around 1 $\mathrm{nm}$, however it should be mentioned that holes in the surface are being found of typically 6-8 $\mathrm{nm}$ in depth.

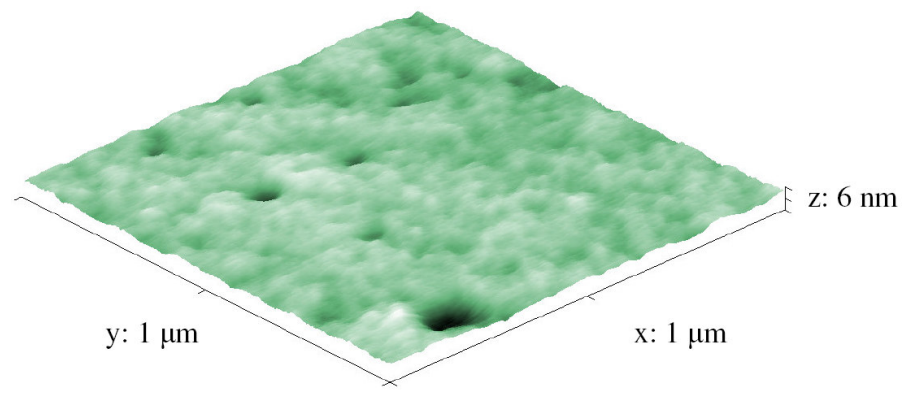

Figure 6-5. A typical AFM scan of the nanoslit bottom. Although the surface roughness has an average values of $1 \mathrm{~nm}$, holes in the surface are being found with depths of around $6 \mathrm{~nm}$. The 3D plot of the scan is stretched in $\mathrm{z}$ direction and thus not on scale with the $\mathrm{x}$ and the $\mathrm{y}$ axis.

To remove the photoresist, the wafer was put in fuming nitric acid $\left(\mathrm{HNO}_{3}\right)$. In order to improve alignment during the next lithography step and the bond alignment step, a $25 \mathrm{~nm}$ chromium layer was deposited. The chromium was sputtered in 1 minute and 40 seconds with 200 Watt DC power at a pressure of $5 \cdot 10^{-3}$ mbar in a home-made system. The next process step is "deep reactive ion etching" (DRIE) and requires a thick layer of photoresist. The resist pattern should also have steep walls to meet the micrometer dimensions of the injection part. Moreover the resist should be baked at high temperature to prevent deformation during DRIE. These demands imply that the usual photolithography procedure is not possible. Therefore an adapted lithography process is used. The adapted process flow for manufacturing the microchannels is depicted in figure 6-6. First a thick resist layer Fujifilm OiR907/35 is spun ( 5 seconds at $500 \mathrm{rpm}$ followed by 20 seconds at $1200 \mathrm{rpm}$ ). After edge bead removal, the wafer is dried $\left(5\right.$ minutes at $\left.95{ }^{\circ} \mathrm{C}\right)$ and baked at high temperature $\left(20\right.$ minutes at $\left.180^{\circ} \mathrm{C}\right)$. At the used conditions, the thickness of the resist is approximately $6.2 \mu \mathrm{m}$. Next a Silspin layer is spun (20 seconds at $2000 \mathrm{rpm})$, dried (1 minute at $\left.95^{\circ} \mathrm{C}\right)$ and baked $\left(3\right.$ minutes at $\left.160{ }^{\circ} \mathrm{C}\right)$. The Silspin layer thickness is now approximately $190 \mathrm{~nm}$. This Silspin is a silicon-rich polymer, which in general is used for planarization in nanoimprinting processes ${ }^{1}$, and is also a good mask for oxygen plasma reactive ion etching. To pattern the Silspin layer, a general photolithography step is used with thin resist to meet micrometer dimensions. This step exists of spin coating of Fujifilm OiR907/12 resist (20 seconds at $6000 \mathrm{rpm})$, drying $\left(1\right.$ minute at $\left.95^{\circ} \mathrm{C}\right)$ and exposure for 4 seconds at $12 \mathrm{mWatt} / \mathrm{cm}^{2}$ through the "microchannel" mask by ElectroVison EVG620 mask aligner in vacuum-hard contact mode at 1.5 bar. Subsequently the resist is baked (1 minute at $95{ }^{\circ} \mathrm{C}$ ) and developed in OPD4262-developer for 40 seconds. In an Elektotech parallel plate reactive ion etcher (RIE), the Silspin is etched for 2 minutes at 10 mTorr with $75 \mathrm{Watt}, 13.56 \mathrm{MHz}$ RF-power and a gas mixture of $25 \mathrm{sccm} \mathrm{CHF}_{3}$ and $10 \mathrm{sccm} \mathrm{O}$. 


\section{The Charge-Patterned Nanoslit}

The substrate table is kept at $10{ }^{\circ} \mathrm{C}$. The thick resist layer is etched in situ for 50 minutes at 10 mTorr with $50 \mathrm{~W} 13.56 \mathrm{MHz}$ RF-power and a gas mixture of $10 \mathrm{sccm} \mathrm{N}_{2}$ and $20 \mathrm{sccm}$ $\mathrm{O}_{2}$. In this step the substrate table is kept at $10^{\circ} \mathrm{C}$ and the generated bias voltage is $200 \mathrm{~V}$. During anisotropic etching of polymers in a reactive ion etcher, a residue is formed on the bottom of the etched pattern. Although this formation is minimized by optimally chosen etch parameters e.g. nitrogen addition and low substrate temperature, a little residue will remain. It was found that this residue can be further reduced by a short chromium etch step, which is a two times one minute wet etch in commercial chromium etch (BASF), with a 10 seconds ultrasonic step in between.

1.

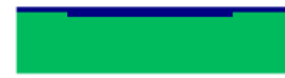

Deposition Cr layer (dark blue)

2.

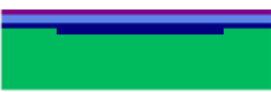

Spincoating Photoresist OiR 907/35 (light blue) and Silspin (purple)

3.

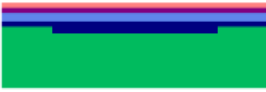

Spincoating OiR 907/12 (pink)

4.

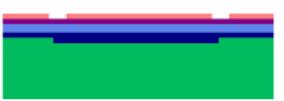

Develop OiR 907/12 (pink)

5.

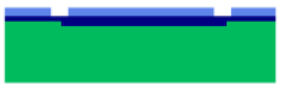

RIE OiR 907/35

6.

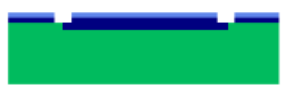

Wet etch $\mathrm{Cr}$

7.

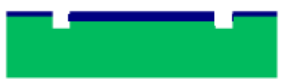

Etching microchannels in Borofloat (DRIE)

8.

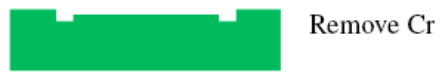

Figure 6-6. The process flow of creating the microchannels in the Borofloat top wafer. 
In figure 6-7 a typical scanning electron microscope (SEM) image is presented of the resist pattern of the injection part of the device. The next fabrication step is the etching of the microchannels. This anisotropical etch process was done with an Adixen AMS100DE during 35 minutes to a channel depth of $20 \mu \mathrm{m}$. A continuous gas flow of $18 \mathrm{sccm} \mathrm{C}_{4} \mathrm{~F}_{8}$, $180 \mathrm{sccm} \mathrm{He}$ and $12 \mathrm{sccm} \mathrm{CH}_{4}$ with 2800 Watt inductive coupled and 350 Watt capacitive coupled RF-power of $13.56 \mathrm{MHz}$ was applied. The used pressure was $8.5 \cdot 10^{-3} \mathrm{mbar}$. The backside of the wafer was $\mathrm{He}$ cooled at $-10^{\circ} \mathrm{C}$. The distance of the wafer to the plasma source was $120 \mathrm{~mm}$. Removal of the resist was done by cleaning the wafer in piranha $\left(\mathrm{H}_{2} \mathrm{SO}_{4}\right.$ and $\mathrm{H}_{2} \mathrm{O}_{2}$ mixture of $2: 1$ at $120^{\circ} \mathrm{C}$ ) for 20 minutes.

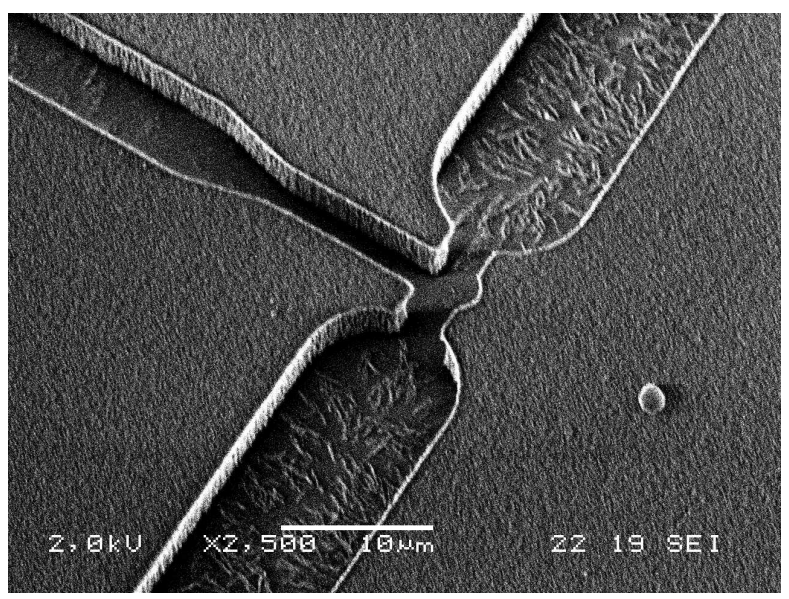

Figure 6-7. A typical scanning electron microscope (SEM) image of the resist pattern of the injection part of the device.

The last fabrication step was powder blasting the access holes which are interfacing the microchannels in the wafer. The photo patternable foil BF410 was used as the mask material. This foil was applied on the backside of the wafer and exposed for 12 seconds at $12 \mathrm{mWatt} / \mathrm{cm}^{2}$ through the "holes" mask by the ElectroVison mask aligner in soft contact mode and the backside alignment option. The protection foil was removed and the wafer was developed for 2 minutes in $0.2 \%$ (w/v) $\mathrm{Na}_{2} \mathrm{CO}_{3}$ solution at $40{ }^{\circ} \mathrm{C}$ with intermediate ultrasonic agitation. For protection of the front side of the wafer during powder blasting, dicing foil was applied. The holes were powder blasted using a home-made setup, with a Comco inc. Microblaster as source. The aluminumoxide powder has a $29 \mu \mathrm{m}$ grain size and the supply air pressure is 5 bar. Both foils were removed by soaking the wafer in acetone.

\subsubsection{The bottom wafer}

In figure 6-8 the total fabrication process of the bottom wafer is presented. The bottom wafer like the top wafer is a $100 \mathrm{~mm}$ Borofloat $33^{\mathrm{TM}}$ wafer from Schott. To create an in- 


\section{The Charge-Patterned Nanoslit}

plane interdigitated pattern of Borofloat and $\mathrm{Al}_{2} \mathrm{O}_{3}$ with a smooth surface, grooves were etched in the Borofloat, filled with the same height of $\mathrm{Al}_{2} \mathrm{O}_{3}$ and as a final step the surface was polished. First the wafer was cleaned by rinsing for 10 seconds in a $25 \% \mathrm{w} / \mathrm{v} \mathrm{KOH}$ solution at $75^{\circ} \mathrm{C}$ and subsequently 10 minutes ultrasonically rinsed in DI water. Then, after a quick dump rinse in DI water, the wafer was immersed in $100 \% \mathrm{HNO}_{3}$ acid for 10 minutes, followed by a second quick dump rinse in DI water. After a dehydration step (10 minutes at $95^{\circ} \mathrm{C}$ ) a HexaMethylDiSilazan (HDMS) layer as adhesion layer was applied on the surface of the wafer by the use of a vapor phase treatment $\left(10\right.$ minutes at $\left.150{ }^{\circ} \mathrm{C}\right)$, which is necessary in order to prevent undercutting during the etching steps.

1 .

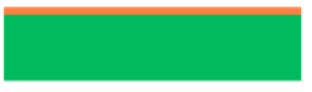

Vapour phase HMDS photo resist OiR 907/12

2.

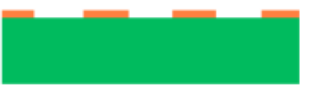

Develop photo resist OiR 907/12

3.

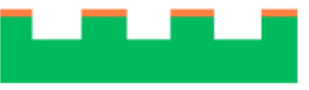

$\mathrm{IBE}+$ Wet etch dip $(\mathrm{BHF} / \mathrm{HCl}, 9: 1)$

4.

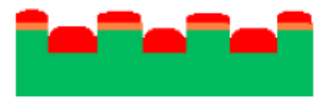

Sputter $\mathrm{Al}_{2} \mathrm{O}_{3}$

5.

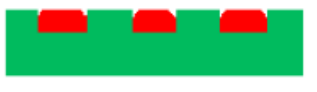

Resist strip and CMP

Figure 6-8. The fabrication process of the bottom wafer.

Fujifilm OiR907/12 photoresist was spin coated (20 seconds at $6000 \mathrm{rpm})$, dried (1 minute at $95{ }^{\circ} \mathrm{C}$ ) and exposed for 2.8 seconds at $12 \mathrm{mWatt} / \mathrm{cm}^{2}$ through the "pattern" mask by the ElectroVison EVG620 mask aligner in vacuum-hard contact mode. Subsequently the resist was baked ( 1 minute at $95{ }^{\circ} \mathrm{C}$ ), developed in OPD4262-developer (40 seconds) and hard baked ( 5 minutes at $95{ }^{\circ} \mathrm{C}$ followed by 5 minutes at $110{ }^{\circ} \mathrm{C}$ ). In this case uniform wafer etching is very important, therefore Ion Beam Etching (IBE) is used instead of DRIE. Wet etching is not possible due to its isotropic properties. The wafer is etched for 44 minutes in a home-made system using an Ion Tech. Inc. MPS-5001 ion source. Argon ions are accelerated by 500 Volt with an intensity of $25 \mathrm{~mA}$, which brings about $132 \mathrm{~nm}$ deep grooves. To improve the lift-off process used further on, a 30 seconds wet etch dip was introduced. This gave a small undercut of $15 \mathrm{~nm}$. The etchant is the same $\mathrm{BHF} / \mathrm{HCl}$ mixture as described before. 
In order to improve alignment during the next lithography step and the bond alignment step, a $25 \mathrm{~nm}$ chromium layer was deposited into the alignment marks. The nanoslit patterns on the wafer were covered by a dummy wafer. Subsequently, aluminum oxide was sputtered in the grooves during 1 minute and 40 seconds with 200 Watt DC power at a pressure of $5 \cdot 10^{-3}$ mbar. The resist is stripped in acetone by ultrasonic agitation for two hours. This will remove the excess aluminum oxide by lift-off. Finally a thorough clean is established in fuming nitric acid for 15 minutes. The final step in the manufacturing of the patterned wafer is polishing the surface by Chemical Mechanical Polishing (CMP) in a Mecapol E460 equipment. Important is the determination of the polish rate. Due to the use of slurries of various concentrations and compositions, residues inside the pads will affect the polishing rate.

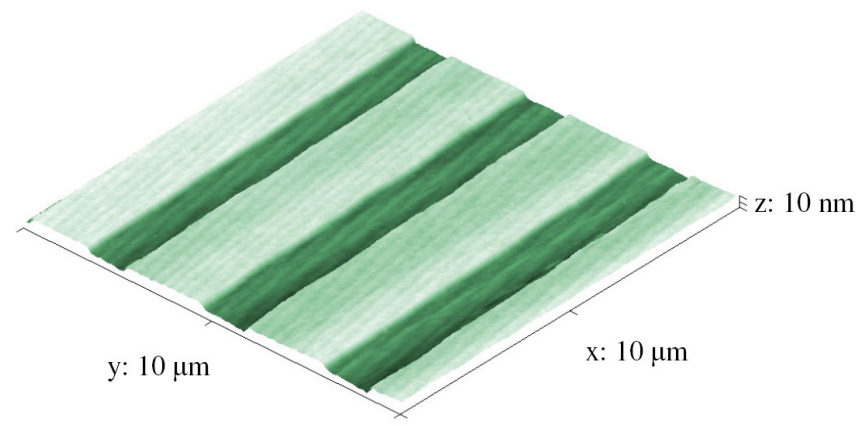

Figure 6-9. An AFM scan of the patterned surface after the polishing step. The $3 \mathrm{D}$ plot of the scan is stretched in the $\mathrm{z}$ direction and thus not on scale with the $\mathrm{x}$ and the $\mathrm{y}$ axis. Between the $\mathrm{SiO}_{2}$ lines (light green), $\mathrm{Al}_{2} \mathrm{O}_{3}$ is situated (dark green). Having the polishing optimized for these pattern dimensions, a minimal difference in height of between $5-10 \mathrm{~nm}$ remained.

Therefore a thorough pre-conditioning step is done by roughening the pad with a diamond grind tool and performing some test runs. In our polish process we used slurry SS25: DI water (1:10). The rotation of the head was $15 \mathrm{rpm}$ and the head pressure $0.4 \mathrm{bar}$, whereas the table speed was $60 \mathrm{rpm}$ and the setup was kept at $20^{\circ} \mathrm{C}$. One polish cycle of 30 seconds will remove about $15 \mathrm{~nm}$ Borofloat. After CMP the wafer was cleaned by 20 min ultrasonic agitation in DI water, followed by a 20 minutes soak in fuming nitric acid. In figure 6-9 a typical AFM scan of the polished structure is presented. The difference in height between the $\mathrm{Al}_{2} \mathrm{O}_{3}$ and the Borofloat lines is between $5-10 \mathrm{~nm}$, which is roughly $10 \%$ of the channel depth $(100 \mathrm{~nm})$. Further optimization might be possible by changing the slurry, because it etches aluminum oxide faster than the Borofloat.

\subsubsection{Wafer bonding and dicing of the device}

Just before bonding the top and bottom wafer are cleaned for $20 \mathrm{~min}$ in fuming nitric acid, followed by 20 min ultrasonic agitation in DI water and finally a quick dump rinse in DI 


\section{The Charge-Patterned Nanoslit}

water. Immediately after spin drying the wafers they are aligned and prebonded. The alignment was done in the EVG620 mask aligner using the anodic bond program.

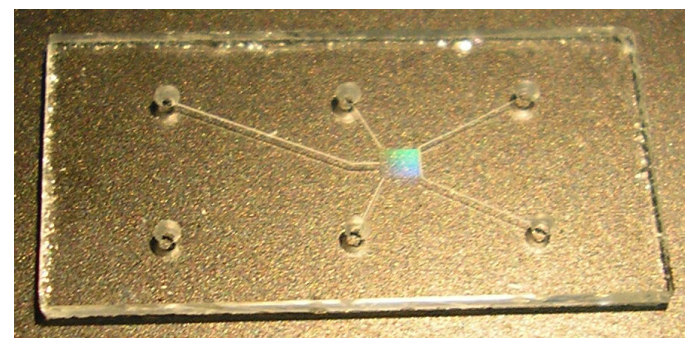

Figure 6-10. A photo of the final device. The blue color in the device is caused by the deflection of the light on the patterned surface.

By rubbing one of the wafers with a plastic tool, the prebond was established. Next, the wafer stack was annealed to increase the bond strength in a programmable furnace, for 1 hour at $625^{\circ} \mathrm{C}$ in air. To prevent cracking, the temperature was ramped slowly, e.g. from $20{ }^{\circ} \mathrm{C}$ to $625^{\circ} \mathrm{C}$ in 6 hours and from $625^{\circ} \mathrm{C}$ to $20^{\circ} \mathrm{C}$ in 12 hours. Finally the wafer stack was diced in a Disco DAD-321 into $10 \times 20 \mathrm{~mm}$ chips, using a $300 \mu \mathrm{m}$ carbon blade. To prevent the chips filling with water during the dice process, the wafer stack was covered by dicing foil. This foil also keeps the channels protected against environmental pollution when stored for a long time. In figure 6-10, the device is presented. The blue color is caused by the deflected light on patterned surface.

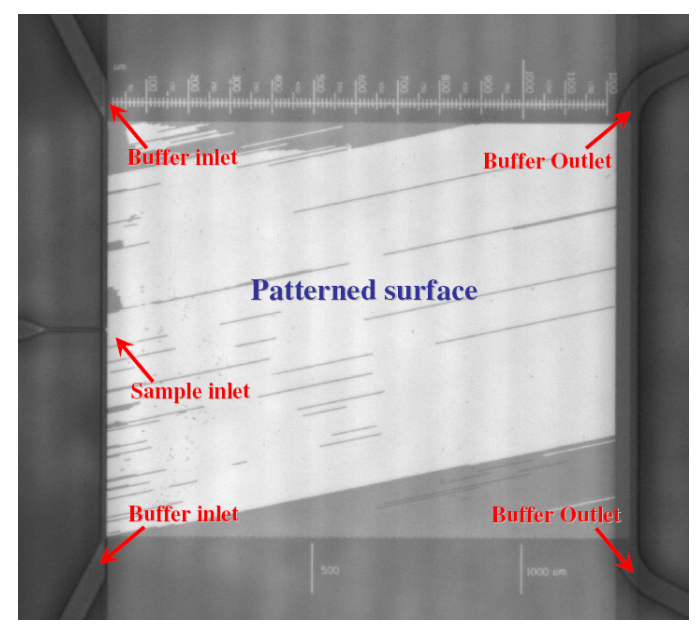

Figure 6-11. The device was filled with $0.1 \mu \mathrm{M} \mathrm{NaAc}$ buffer $(\mathrm{pH}=5)$. The stripes on the patterned surface appeared after the removal of the protection foil which covered the inlets and outlets during the dicing of the device. 


\subsubsection{Filling of the device}

We were able to successfully fill the device with a $0.1 \mathrm{mM} \mathrm{NaAc}$ buffer $(\mathrm{pH}=5)$. In figure 6-11 an image is presented of the first filling experiment. In this image, at some places the stripes of the pattern are visible. This occurred after the removal of the protection foil which covered the inlets and outlets during the dicing of the device.

\subsection{Conclusions}

We developed a fabrication procedure for the manufacturing of a surface-patterned nanoslit device. The pattern is composed of $\mathrm{Al}_{2} \mathrm{O}_{3}$ and $\mathrm{SiO}_{2}$ (Borofloat), which were deposited and polished using CMP techniques. The device was anodically bonded using a temperature of $625^{\circ} \mathrm{C}$. We have been able to successfully realize devices with a height of $100 \mathrm{~nm}$. When using for example a $0.1 \mathrm{mM} \mathrm{NaAc}(\mathrm{pH}=5)$, the surface charge is expected to be alternatingly positive $\left(\mathrm{Al}_{2} \mathrm{O}_{3}\right)$ and negative $\left(\mathrm{SiO}_{2}\right)$. The device could be successfully filled with this buffer by using a high pressure pump (Harvard Apparatus, PHD 2000).

\subsection{References}

1. M. Miller, G. Doyle, N. Stacey, F. Xu, S. V. Sreenivasan, M. Watts, D. L. LaBrake, "Fabrication of nanometer sized features on non-flat substrates using a nano-imprint lithography process," Molecular Imprints Inc., Austin Texas, 2005. 


\section{The Charge-Patterned Nanoslit}

\section{Appendix}

Below an example of a device is presented. The maximum number of devices which fits on a 4 inch wafer is 21 . Each device is $1 \times 2 \mathrm{~cm}$ (length $\mathrm{x}$ width). Among all designs we varied the pattern line dimensions (line width $\mathrm{x}$ distance between lines), the line angle, the nanoslit dimensions and the injection type (see section 6.2, figure 6-2). Since the manufacturing of this device is done by normal photolithography, the line dimensions were chosen according to the possibilities of the used manufacturing techniques. An overview of the designs is presented on the next page. The depth of the nanoslits can be defined according to the users' whishes, however it will be very difficult when working with pressure driven flow below $100 \mathrm{~nm}$.

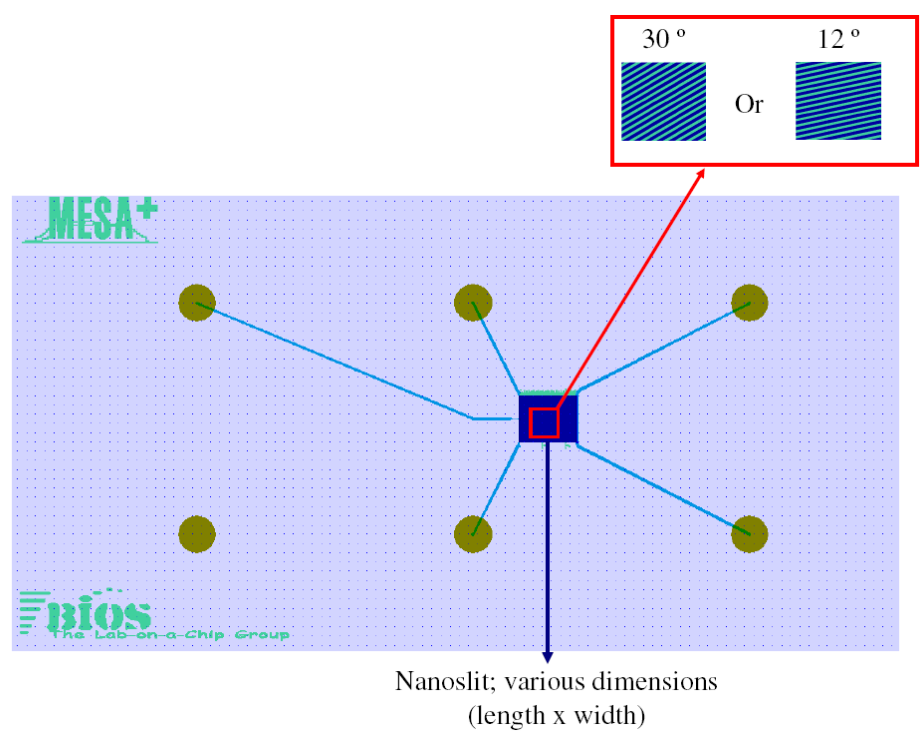

Below an example of a device is depicted as presented in the overview on the next page with an explanation next to it.

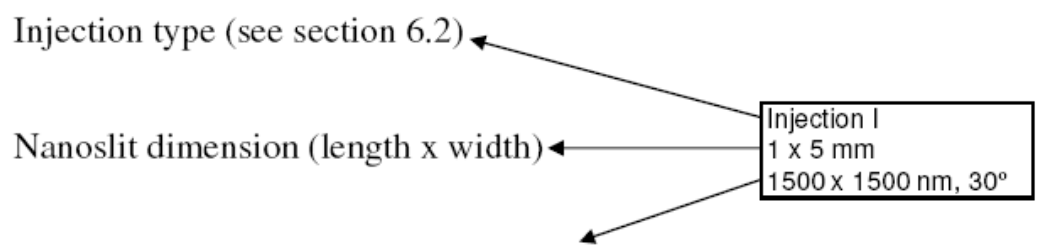

Line dimensions of the pattern (width line, interdistance of lines), angle of the lines $\left(12^{\circ}\right.$ or $\left.30^{\circ}\right)$ 


\section{Chapter 6}

An overview of the different device designs.

\begin{tabular}{|l|}
\hline Injection I \\
$1 \times 10 \mathrm{~mm}$ \\
$1500 \times 2000 \mathrm{~nm}, 12^{\circ}$
\end{tabular}

Injection

$1 \times 5 \mathrm{~mm}$

$1500 \times 2000 \mathrm{~nm}, 12^{\circ}$

Injection I

$1 \times 1.2 \mathrm{~mm}$

$1500 \times 2000 \mathrm{~nm}, 12^{\circ}$

Injection I

$1 \times 1.2 \mathrm{~mm}$

$1500 \times 1500 \mathrm{~nm}, 30^{\circ}$

Injection I
$1 \times 1.2 \mathrm{~mm}$
$1500 \times 1500 \mathrm{~nm}, 12^{\circ}$

Injection I

$1 \times 5 \mathrm{~mm}$

$1500 \times 1500 \mathrm{~nm}, 30^{\circ}$

Injection I
$1 \times 5 \mathrm{~mm}$
$1500 \times 1500 \mathrm{~nm}, 30^{\circ}$

\begin{tabular}{|l|}
\hline Injection II \\
$1 \times 10 \mathrm{~mm}$ \\
$1500 \times 2000 \mathrm{~nm}, 12^{\circ}$ \\
\hline
\end{tabular}

\begin{tabular}{|l|}
\hline Injection II \\
$1 \times 5 \mathrm{~mm}$ \\
$1500 \times 2000 \mathrm{~nm}, 12^{\circ}$ \\
\hline
\end{tabular}

\begin{tabular}{|l|}
\hline Injection II \\
$1 \times 1.2 \mathrm{~mm}$ \\
$1500 \times 2000 \mathrm{~nm}, 12^{\circ}$ \\
\hline
\end{tabular}

\begin{tabular}{|l|}
\hline Injection II \\
$1 \times 1.2 \mathrm{~mm}$ \\
$1500 \times 1500 \mathrm{~nm}, 30^{\circ}$ \\
\hline
\end{tabular}

\begin{tabular}{|l|}
\hline Injection II \\
$1 \times 1.2 \mathrm{~mm}$ \\
$1500 \times 1500 \mathrm{~nm}, 12^{\circ}$ \\
\hline
\end{tabular}

\begin{tabular}{|l|}
\hline Injection II \\
$1 \times 5 \mathrm{~mm}$ \\
$1500 \times 1500 \mathrm{~nm}, 30^{\circ}$ \\
\hline
\end{tabular}

\begin{tabular}{|l|}
\hline Injection II \\
$1 \times 5 \mathrm{~mm}$ \\
$1500 \times 1500 \mathrm{~nm}, 30^{\circ}$
\end{tabular}
Injection II

$1 \times 5 \mathrm{~mm}$

$1500 \times 2000 \mathrm{~nm}, 12^{\circ}$

Injection I

$1 \times 5 \mathrm{~mm}$

$1500 \times 2000 \mathrm{~nm}, 12^{\circ}$

Injection II

$1 \times 1.2 \mathrm{~mm}$

$1500 \times 2000 \mathrm{~nm}, 12^{\circ}$

Injection II

$1 \times 1.2 \mathrm{~mm}$

$1500 \times 1500 \mathrm{~nm}, 30^{\circ}$

Injection II

$1 \times 1.2 \mathrm{~mm}$

$1500 \times 1500 \mathrm{~nm}, 12^{\circ}$

Injection I

$1 \times 5 \mathrm{~mm}$

$1500 \times 1500 \mathrm{~nm}, 30^{\circ}$

Injection I

$1 \times 5 \mathrm{~mm}$

$1500 \times 1500 \mathrm{~nm}, 30^{\circ}$ 
Filename: 6_Patterned nanoslits_For_WPS_Final_Reserve Directory: $\quad$ C:Locuments and SettingslGeorgette.UTWENTEA174A94\Desktop\Ready for printing $\backslash$ Reserve Template: C:Documents and SettingslGeorgette.UTWENTEA174A94\Application DatalMicrosoft|Templates $\backslash$ Normal.dot Title: 1

Subject:

Author:

Keywords:

Comments:

Creation Date: $\quad$ 9/29/2009 12:02:00 PM

Change Number: 2

Last Saved On: $\quad$ 9/29/2009 12:02:00 PM

Last Saved By: Georgette

Total Editing Time: 1 Minute

Last Printed On: $\quad$ 9/29/2009 12:02:00 PM

As of Last Complete Printing

Number of Pages: 14

Number of Words: $\quad$ 4,038 (approx.)

Number of Characters: 19,064 (approx.) 


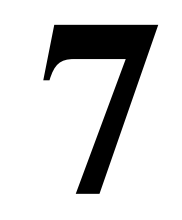

\section{Conclusions and Perspectives}

In this chapter the conclusions of the work presented in this thesis are summarized and compared with the project aims. Next to this, aspects are mentioned which could be useful for the improvement of further investigations and suggestions for further research are given. 


\section{Chapter 7}

\subsection{Summary}

In this thesis the results of fundamental investigations of the electrokinetic transport of $\lambda$ DNA and Litmus DNA (XbaI digested) in nanoslits is described. Fused silica nanoslit devices of different height were used for the experiments. In the last chapter we furthermore described a new type of device which could offer new possibilities for both fundamental investigations and separation applications.

In order to understand the DNA separation using nanostructures, the main theories developed in gel electrophoresis were discussed in chapter 2. Examples of important theories and models are "biased reptation", Ogston sieving and entropic trapping. It is shown that these models are also valid when using nanostructures. Clearly, it is important to perform further fundamental and/or separation investigations of DNA or other biopolymers in nanostructures. The huge advantage of these nanostructures with respect to gels is the exact manufacturing control of dimensions.

The electrokinetic transport of $\lambda$-DNA when DC electrical fields are applied $(6-200$ $\mathrm{kV} / \mathrm{m}$ ), was studied in fused silica nanoslit devices and reported in chapter 3 . The $\lambda$-DNA molecules showed a field dependent mobility. Next to this, it was shown that the $\lambda$-DNA molecules followed preferential pathways through the nanoslits. The increasing mobility up to a DC field strength of $30 \mathrm{kV} / \mathrm{m}$ and the observation of preferential pathways can both be explained by the biased reptation model. At electrical DC fields strengths of $30 \mathrm{kV} / \mathrm{m}$ and below the molecules moved in a fluent way, however above field strengths of $30 \mathrm{kV} / \mathrm{m}$ this fluent movement changed in an intermittent movement and trapping of $\lambda$-DNA molecules. The $\lambda$-DNA molecules were found to be transiently trapped at specific places whereafter they continued moving. It was clearly shown that the higher the applied DC electrical field, the more $\lambda$-DNA molecules were trapped. The transport behavior of the smaller Litmus DNA (XbaI digested) was also investigated. This molecule also showed a field dependent mobility which was lower than that of the $\lambda$-DNA molecules. Litmus DNA only moved in an intermittent way through the nanoslits at all applied electrical DC fields. This difference in mobility between the two molecules of different length opens the door to the development of new separation devices. For the intermittent movement and the trapping we suggested a mechanical and/or a dielectrophoretic mechanism.

The hypothesis of a possible dielectrophoretic trapping mechanism was first investigated and is reported in chapter 4 . For this purpose the electrokinetic transport of the $\lambda$-DNA molecules was analyzed when AC electrical fields $(10-200 \mathrm{kV} / \mathrm{m} ; 100 \mathrm{~Hz}$ and $1 \mathrm{kHz})$ were superimposed onto DC electrical fields $(10-200 \mathrm{kV} / \mathrm{m})$. For a larger part of the data, no significant influence of the superimposed AC field was found. When a significant influence was found, it was apart from some exceptions, in most cases an increase in mobility in particular at the low DC fields. Therefore the main body of the measurements indicates that dielectrophoretic phenomena are not important. In this investigation we found both fluent and intermittent movements of the $\lambda$-DNA molecules at different AC and DC field strengths. Apart from this, we also found preferential pathways. Again the 


\section{Conclusions and Perspectives}

increase in mobility we found with increasing AC field strength at low DC fields can be explained by the biased reptation model, assuming the AC fields cause an increased orientation of the DNA molecules in the field direction.

In order to further investigate the mechanism behind the intermittent movement of the $\lambda$ DNA molecules, the electrokinetic transport behavior was investigated using fused silica nanoslits with heigths of $60 \mathrm{~nm}$ and $120 \mathrm{~nm}$. The measurement results are reported in chapter 5. Only in the $60 \mathrm{~nm}$ high nanoslits some intermittent movement was seen, but merely for a small percentage of the $\lambda$-DNA molecules whereas most of the molecules moved fluently through the nanoslit. The mobility of $\lambda$-DNA was furthermore independent of the field strength and no preferential pathways were observed. Interestingly, the mobility at a DC field of $30 \mathrm{kV} / \mathrm{m}$ decreased in the order $20 \mathrm{~nm}>60 \mathrm{~nm}>120 \mathrm{~nm}$. We currently have no explanation for this observation. Next to the $\lambda$-DNA molecules, Litmus DNA (XbaI digested) was also investigated. We were not able to track these molecules inside the nanoslits at any applied DC electrical field, however at DC fields of $2 \mathrm{MV} / \mathrm{m}$ the molecules were found to be trapped and enriched at certain positions inside the nanoslits

For the investigation of the separation possibilities using nanoslits, a charged patterned nanoslit device was manufactured, as described in chapter 6. The use of two different materials patterned on a single surface in stripes, namely $\mathrm{Al}_{2} \mathrm{O}_{3}$ and borofloat glass, is expected to result in two oppositely charged surface parts when used at specific $\mathrm{pH}$ conditions. Together with an adjustable double layer thickness, the influence of the surface charge on biomolecular behavior and separation in such a nanoslit device can be investigated. This device was manufactured of two bonded borofloat wafers. In one wafer the nano- and microchannels are manufactured, whereas in the other wafer the charge patterned surface is manufactured. First a pattern was etched into the borofloat glass surface by the use of standard photolithography, which was followed by the deposition $\mathrm{Al}_{2} \mathrm{O}_{3}$ and the polishing of the surface. Finally the patterned surface was scanned by AFM, wafers were bonded and diced. The device was tested by filling the nanoslit with a $0.1 \mathrm{mM}$ $\mathrm{NaAc}(\mathrm{pH}=5)$ buffer and YOYO-1 labeled $\lambda$-DNA molecules.

\subsection{Outlook}

Based on the results described in this thesis, it can be concluded that nanoslit devices are extremely useful tools for investigating the electrokinetic transport of DNA molecules. By using these devices, significant new knowledge was obtained on the fundamentals of electrokinetic transport of DNA.

The experiments described in this thesis can be improved and/or adapted in a variety of ways to obtain better results. Below several issues are discussed.

First of all, a very important improvement of the quality of the obtained video movies could be provided by a better cooling of the EMCCD camera. This type of camera has an 
internal cooling system, however we noticed that it is very difficult to maintain temperatures below $-65{ }^{\circ} \mathrm{C}$ during an experiment. This typically results in a high background noise in the recorded video movies. An improvement could be provided in two different ways: either by a better air circulation in the experimental space, or by attaching the camera to a water cooling system. For the final experiment described in chapter 5 , the microscope setup was already replaced and a better cooling of the camera was provided, despite which the maintenance of the temperature still remained difficult.

Additionally, pointing at the performance of the camera, it is worth to investigate the positional error caused by the finite exposure time of the camera while recording the movements of the single molecules. This could be done for example by small bright molecules or fluorescent nanoparticles. By recording the movements at several velocities and studying the fluorescent signal resulting from streaking one can estimate the positional error introduced when long DNA molecules are studied. In this way additional information could be extracted from the recorded video movies.

As was presented in this thesis, the fundamental studies of DNA molecules in nanoslits resulted in unexpected results. To further investigate these results, suggestions will be given according to the chapters.

\subsubsection{DC field dependent DNA mobility in $20 \mathrm{~nm}$ high nanoslits}

In this chapter we saw that both the $\lambda$-DNA and the Litmus DNA (XbaI digested) molecules have a field dependent mobility. Furthermore we saw that Litmus DNA was transported exclusively in an intermittent way in all applied DC fields, whereas the $\lambda$-DNA molecules were transported fluently in DC fields until a strength of $30 \mathrm{kV} / \mathrm{m}$ and intermittently in the DC fields above $30 \mathrm{kV} / \mathrm{m}$. It would be very interesting to investigate a larger size range of DNA molecules in a larger DC field range. This could provide more information about the "Stop and Go" behavior and whether this behavior can be used to separate DNA molecules on length from each other. One possibility already indicated by the present experiments could be the separation of small DNA molecules such as Litmus DNA from $\lambda$-DNA by the use of low DC fields. The larger mobility and the fluent transport of the $\lambda$-DNA molecules could enable a fast separation of $\lambda$-DNA from Litmus DNA.

\subsubsection{AC/DC field dependent DNA mobility in $20 \mathrm{~nm}$ high nanoslits}

In this chapter we investigated the influence of superimposed AC fields on DC fields on the trapping behavior of the $\lambda$-DNA molecules. Next to a clear increase in mobility which we found for the applied DC fields below $20 \mathrm{kV} / \mathrm{m}$ at all superimposed AC fields $(1 \mathrm{kHz})$, the results depended in a very complicated way on applied electric fields. A few measurements showed a mobility decrease instead of an increase which observations need to be further investigated. I recommend further investigations at a wider frequency range of 


\section{Conclusions and Perspectives}

the AC fields (for example $5 \mathrm{~Hz}$ till $1 \mathrm{kHz}$ ), superimposed on a wider range of applied DC fields $(10 \mathrm{kV} / \mathrm{m}-500 \mathrm{kV} / \mathrm{m})$. Next to this, it would be very interesting to study the behavior of Litmus DNA molecules (XbaI digested) as well. Since this smaller molecule was transported only in an intermittent way at DC fields, the superimposition of an AC field could provide additional information about the "Stop \& Go" behavior.

\subsubsection{DNA mobility in $60 \& 120 \mathrm{~nm}$ high nanoslits at applied DC fields}

In this chapter we presented the investigation into the transport behavior of $\lambda$-DNA molecules in $60 \& 120 \mathrm{~nm}$ nanoslits and compared this with the results obtained in the 20 $\mathrm{nm}$ nanoslits. Surprisingly we found a decrease in mobility with an increase of channel height. It would be very interesting to investigate a larger range of DNA sizes and measure the mobilities. If the mobility remains constant at different applied fields, however changes with length, than this could lead to the development of a new type of separation device. Next to this, it would also be of interest to investigate much larger molecules than $\lambda$-DNA, to see whether preferential pathways could be found and if so then whether there is a relation to the field dependent mobility. This could confirm the hypothesis of the occurrence of biased reptation of DNA molecules in nanoslits.

\subsubsection{The charge patterned nanoslit}

In this chapter the manufacturing of this device is described. Unfortunately, due to a lack of time, experiments could not be performed with this device. With this device we planned to investigate the capabilities of a charge patterned double layer landscape inside a nanoslit while pumping a buffer flow with small labeled DNA molecules through it. Suggestions for further research include the use of different concentrations of $\mathrm{NaAc}$ buffer $(\mathrm{pH}=5)$ and to first study the transport of YOYO-1 labeled Litmus DNA molecules (both circular and $\mathrm{Xbal}$ digested). If it can be shown that the molecules flow in a preferential way through the nanoslit, other DNA lengths can be investigated to see whether such a pattern can offer new separation possibilities on length and charge. 
Chapter 7 
Filename: 7_Conclusions and prospectives_For_WPS_Final_Reserve Directory: C:LDocuments and SettingsiGeorgette.UTWENTE-

A174A94\Desktop\Ready for printing $\backslash$ Reserve

Template:

C:Documents and SettingslGeorgette.UTWENTE-

A174A94\Application DatalMicrosoft|Templates $\backslash$ Normal.dot

Title: 1

Subject:

Author:

Georgette

Keywords:

Comments:

Creation Date: $\quad$ 9/29/2009 11:55:00 AM

Change Number: 2

Last Saved On: $\quad$ 9/29/2009 11:55:00 AM

Last Saved By: Georgette

Total Editing Time: 0 Minutes

Last Printed On: $\quad$ 9/29/2009 11:56:00 AM

As of Last Complete Printing

Number of Pages: 6

Number of Words: $\quad$ 2,074 (approx.)

Number of Characters: 9,791 (approx.) 
Appendix 1

\section{List of Abbreviations and Symbols}


Appendix 1

\section{List of abbreviations and symbols}

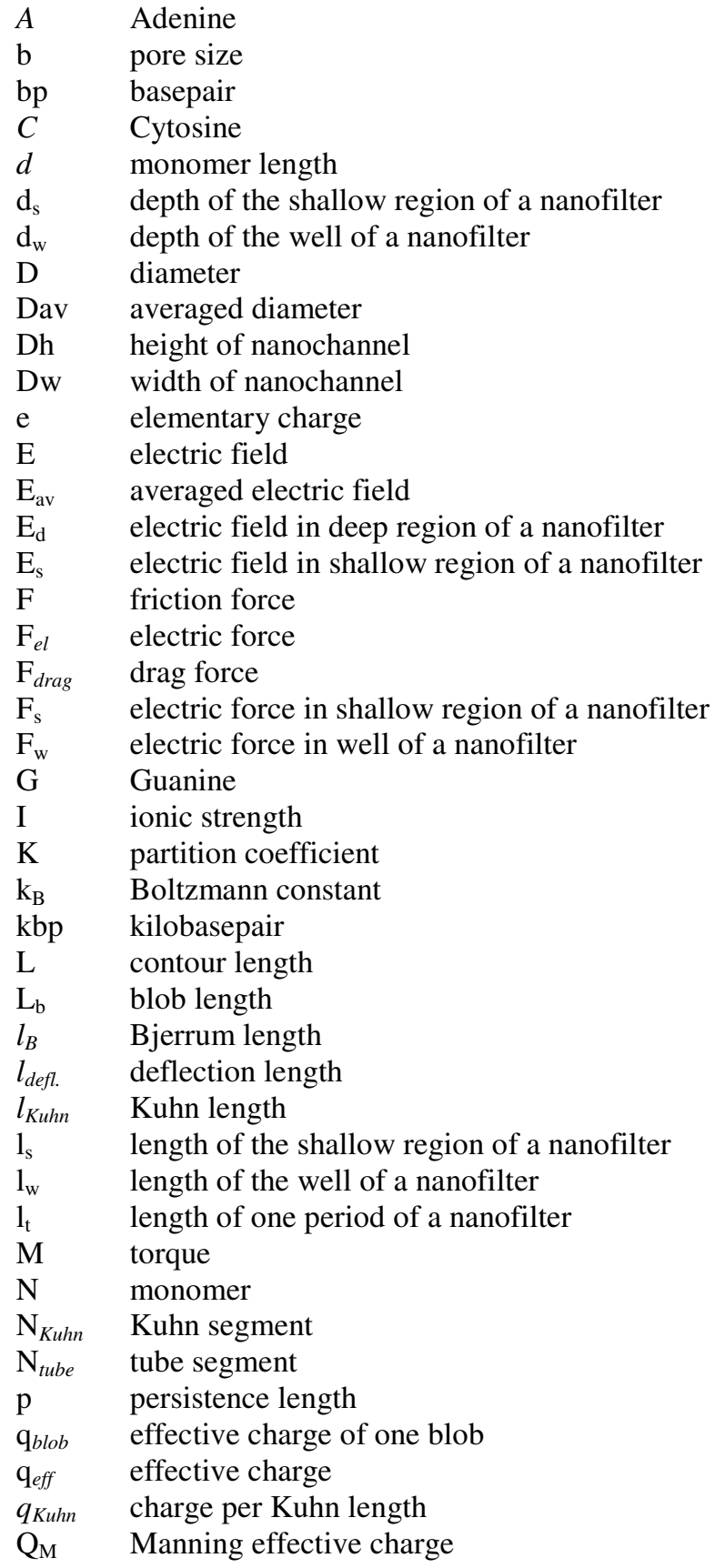


List of Abbreviations and Symbols

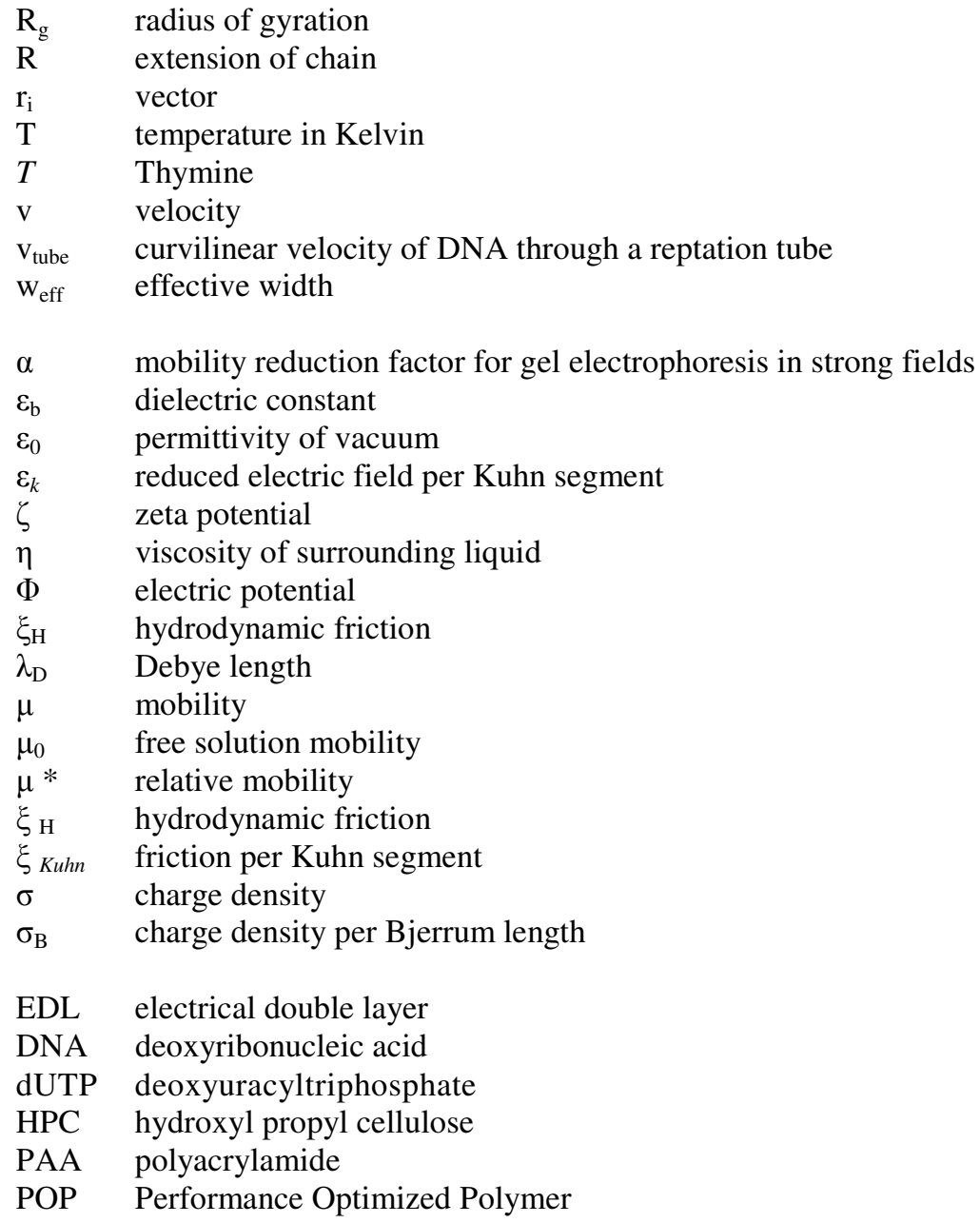


Appendix 1 
Filename: Appendix 1_Final_For_WPS_Final_Reserve Directory: $\quad$ C:Locuments and Settings/Georgette.UTWENTEA174A94\Desktop\Ready for printing $\backslash$ Reserve Template: C:LDocuments and SettingslGeorgette.UTWENTEA174A94\Application DatalMicrosoft|Templates\Normal.dot Title: 1

Subject:

Author:

Keywords:

Comments:

Creation Date: $\quad$ 9/29/2009 11:50:00 AM

Change Number: 2

Last Saved On: $\quad$ 9/29/2009 11:50:00 AM

Last Saved By: Georgette

Total Editing Time: 1 Minute

Last Printed On: $\quad$ 9/29/2009 11:50:00 AM

As of Last Complete Printing

Number of Pages: 4

Number of Words: 348 (approx.)

Number of Characters: $\quad 1,643$ (approx.) 


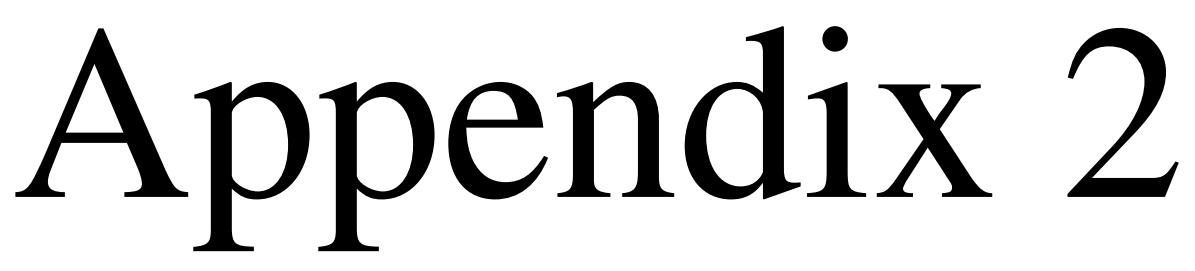

\section{Single DNA Molecule Experiments}

In this Appendix we present the experimental conditions, used for the single DNA molecules experiments in the nanoslits, which are not varied in the presented investigations of this thesis. 


\section{A.2.1. Introduction}

In this thesis, experiments with single DNA molecules in nanoslits are performed. In all described investigations in this thesis, the design, material and the manufacturing of the device remain the same. Next to this, the chip-holder, the used microscope setup, EMCCD camera and the buffer compositions are not varied. Therefore, this will be presented in this chapter. The experimental conditions which were varied, such as the batch of the devices, height of the nanoslits, applied electrical field in DC or AC/DC will be described in the experimental protocol prior to each presented investigation. Also, in this experimental protocol, a typical AFM scan of the used batch of devices will be presented. In this chapter, we will start with the manufacturing of nanoslit device, followed by describing the experimental setup and finally the fluorescent labelling of DNA molecules and the composition and preparation of the buffers. Special attention will be given to the used EMCCD camera, the fluorescent label YOYO-1 and the composition of the buffer.

\section{A.2.2 The nanoslit device}

The nanoslit devices were manufactured in fused silica (figure A2-1). The dimensions of the chip are $10 \mathrm{~mm} \times 20 \mathrm{~mm}$, and it is composed of two fused wafers.

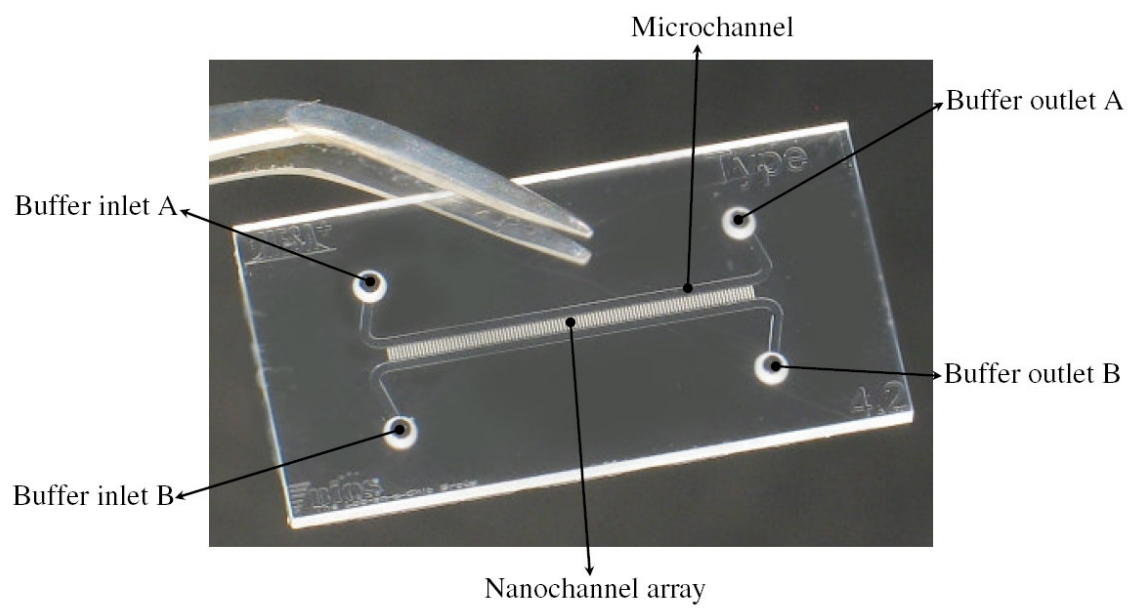

Figure A.2-1 Top view of the fused silica device. Between the microchannels an array of 100 nanoslits is situated. Each nanoslit is facing at both sides the microchannels. The dimensions of the nanoslits are $3 \mu \mathrm{m}$ in width, $500 \mu \mathrm{m}$ in length and a height of 20,60 or $120 \mathrm{~nm}$.

In one wafer an array of hundred nanoslits are manufactured by standard photolithography and etching in a buffered hydrogen fluoride (BHF) solution. The dimension of each nanoslit is $3 \mu \mathrm{m}$ in width, $500 \mu \mathrm{m}$ in length and 20, 60 or $120 \mathrm{~nm}$ in height. In the other wafer, two microchannels are manufactured by using standard photolithography and 


\section{Single DNA Molecule Experiments}

etching with $0.5 \% \mathrm{HF}$ solution. The dimensions of the microchannels are $19 \mu \mathrm{m}$ in height, $300 \mu \mathrm{m}$ in width and $12.5 \mathrm{~mm}$ in length. The four fluidic connection holes inside the device were manufactured by photolithography and powder blasting. The surface roughness in the nanoslits was also studied for each batch of devices manufactured. A typical AFM scan is shown in figure A2.-2. AFM tips were used with a radius of $7 \mathrm{~nm}$ and $2 \mathrm{~nm}$. The rms roughness values found were ranging from $0.8 \mathrm{~nm}-1.4 \mathrm{~nm}$. Holes in the surface, with a depth down to $8 \mathrm{~nm}$ also occur as can be seen in the AFM scan shown in figure A2-2. The holes were already present before etching (AFM scans). In each experimental chapter of this thesis, a typical AFM scan of the used device will be shown. After the AFM measurements the wafers were oxygen plasma cleaned and fusion bonded at a temperature of $1100{ }^{\circ} \mathrm{C}$. The bonding creates a connection between the microchannels by the intermediate array of nanoslits.

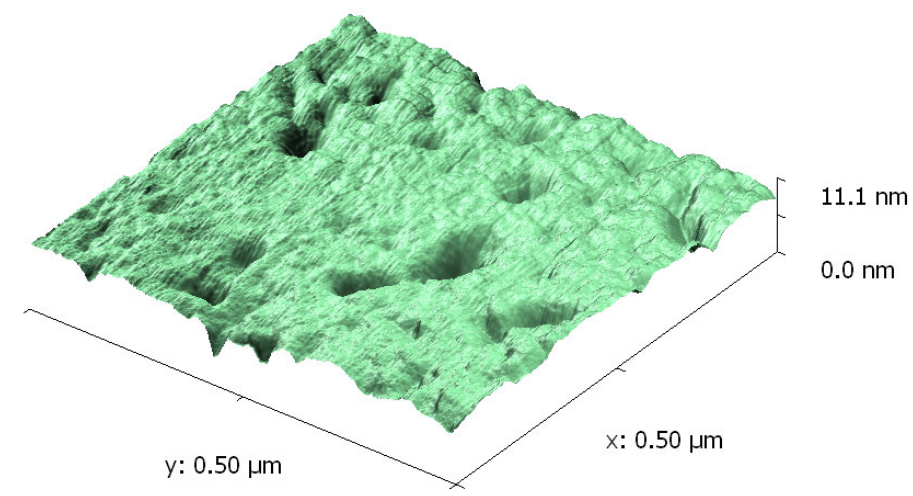

Figure A.2-2. An example of the surface roughness of the $20 \mathrm{~nm}$ high nanoslits as measured by AFM and using tips with a radius of $2 \mathrm{~nm}$. The rms value is $0.8-1.4 \mathrm{~nm}$. In the surface, holes occur with a depth down to $8 \mathrm{~nm}$. This device is from a batch of devices used in the investigation presented in chapter 3 .

\section{A.2.3 The experimental setup}

The nanoslit devices are used together with a home-made chipholder (see also figure A.23 ). In this chipholder it is possible to screw connectors (Upchurch), which enables us to connect to the holes in the device with the syringes and the buffer-vials via capillaries (Inacom) as presented in figure A2-4. The buffer in the syringes is continuously pumped during the experiments through the microchannels of the device to the buffer-vials. To apply electrical fields over the array of nanoslits, Pt electrodes are placed in the buffervials. The applied fields in $\mathrm{DC}$ or AC/DC vary for each investigation and will therefore further explained in the experimental protocols of each presented investigation of this thesis. To study the single $\lambda$-DNA molecules inside the nanoslits, we use an epifluorescence microscope (Leica) and a specific filter cube (L5) which allows us to detect 
Appendix 2

the fluorescent label used. The L5 filter cube exists of a dichroic mirror and an excitation and emission filter.

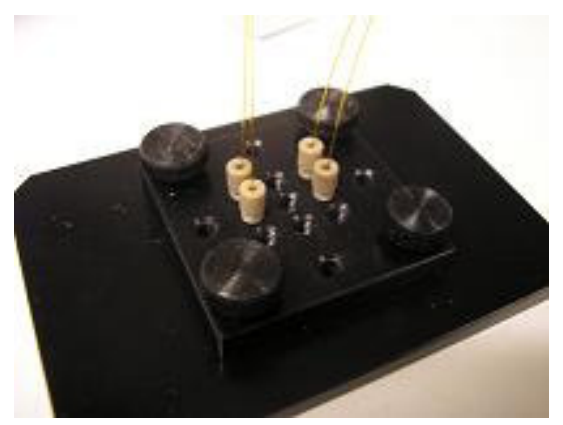

Figure A.2-3. The chipholder in which the nanoslit device is fixed. The connectors can be screwed into the chipholder and connect the device to the syringes and buffer vials.

The mercury lamp (see figure A.2-4) is illuminating the filter cube of the microscope. The excitation filter (orange bar in the filter cube of figure A2-4) will only let the light through which is close to the excitation wavelength of the fluorescent label. This light illuminates the fluorescent labelled $\lambda$-DNA molecules in the nanoslits after passing the objective (63x). The fluorescent light emitted by the label can pass both the dichroic mirror and the emission filter in the filter cube (blue and green bar respectively in the filter cube presented in figure A.2-4). Since we are performing single molecule measurements which are providing weak fluorescent signals, the sensitivity of the camera is of high importance. Therefore, an electron multiplying CCD camera is used (Andor iXon EMCCD). This type of camera can detect and multiply very weak signals. However, even single thermally generated electron in the silicon of photosensitive device will be amplified, resulting in the final signal as a noise. This type of noise, referred as dark current contribution, can be reduced by effective cooling of the camera. The camera used, has an inferior cooling system. During the experiments, the camera was cooled until $-80^{\circ} \mathrm{C}$. We noticed that circulation of air in the environment of the camera is very important to maintain this low temperature and therefore an additional air-condition in the laboratory was used. The video frames were recorded with the frame transfer option of the software. In this way, we were allowed to record frames directly after each other. To control the settings of the camera and to acquire the video frames during the experiments the program Andor Solis was used. 


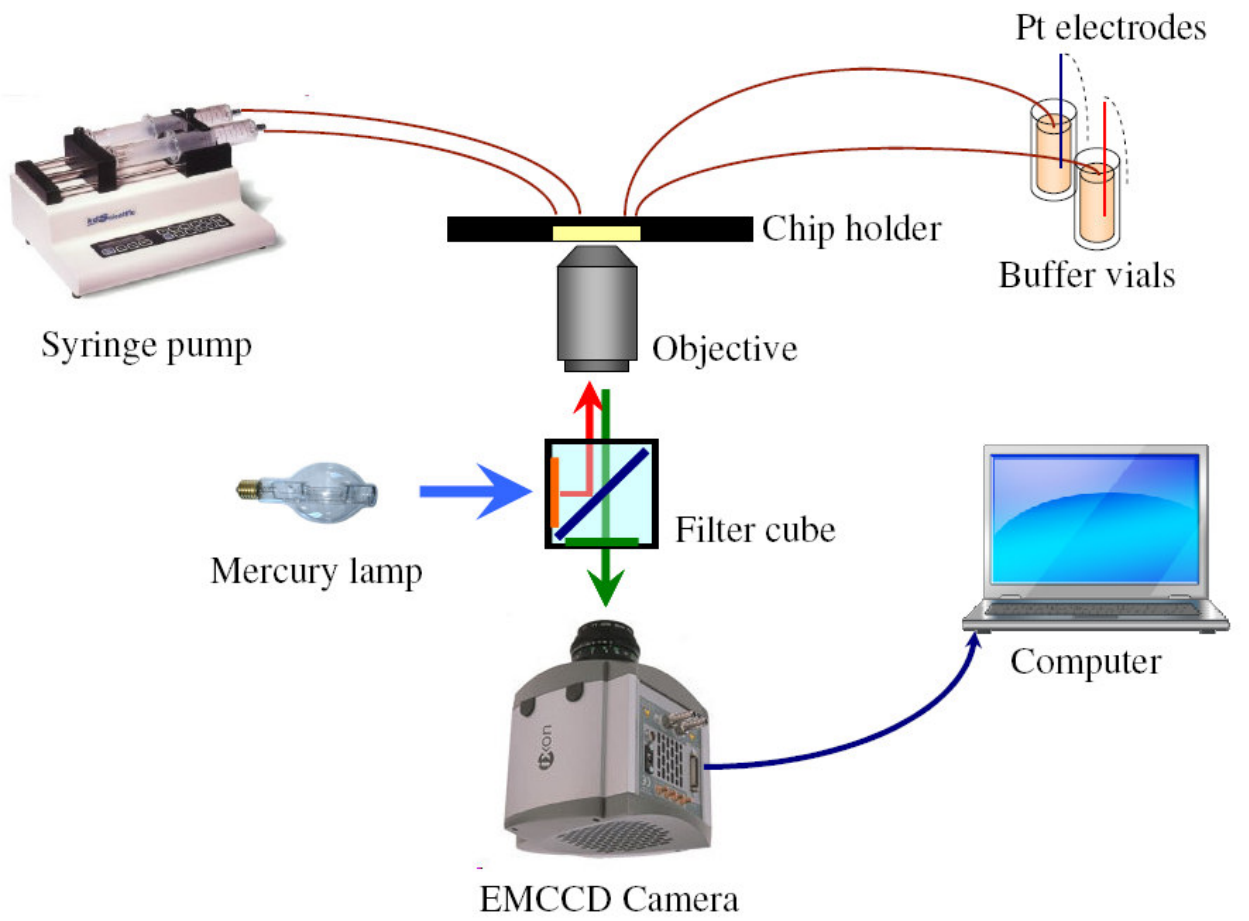

Figure A.2-4. The mercury lamp sends light to the filter cube.The light close to the excitation wavelength of the fluorescent label is filtered by the excitation filter (orange bar in the filter cube) and send through the objective $(63 \mathrm{x})$ to the $\lambda$-DNA molecules. The emitted fluorescent signal is selected by the emission filter and is detected by the EMCCD camera and subsequently send to the computer. During the experiments, buffer is pumped through the microchannels.

\section{A.2.4 Electrical potential measurement}

Calculation shows that the resistance of the microchannels is negligible with respect to the resistance of the nanochannels. If we use the voltage information that is obtained from the used voltage source, than a small error is made resulting from the Pt electrodes. The Pt electrodes are inert and will only transfer electrons when an electrical field is applied. Thus, there must be a redox reaction in the buffer which will start when the applied electrical field reached a specific strength. To measure the exact applied potential difference over the nanoslit array, a potentiostat (Parstat 2263, Princeton Applied Research) and $\mathrm{Ag} / \mathrm{AgCl}$ reference electrodes were used which were placed in the buffer vials. 
Appendix 2

\section{A.2.5 Sample preparation}

In this paragraph, the used buffer, fluorescent dye and additional chemicals will be discussed. At the end of the paragraph a description of the sample preparation is given.

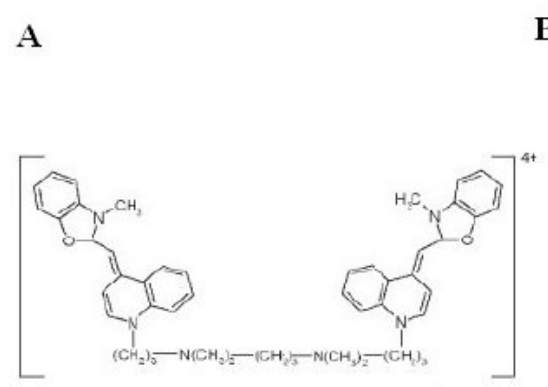

B

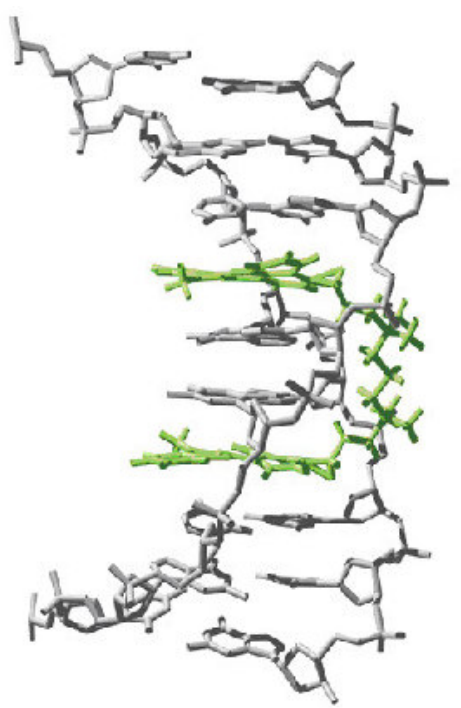

Figure A.2-5. In A, the chemical structure of the intercalating dye YOYO-1 is presented. The molecule has a total charge of $4^{+}$. B presents the analogue of YOYO-1, TOTO-1 (green) intercalated between the basepairs of a DNA molecule (grey). In TOTO-1 the Oxygen atom is replaced by a Sulphur atom. Picture with permission used from: www.invitrogen.com

For all the experiments, $1 \mathrm{x}$ Tris-Borate-EDTA (TBE) buffer was used. This buffer has a low conductivity and is commonly used with DNA electrophoresis in gels and DNA diagnostics ${ }^{1}$. The EDTA is a chelating agent inside the buffer, captures multivalent ions as $\mathrm{Mg}^{2+}$ out of the buffer solution. These ions are co-factors of many enzymes, therefore capturing will help to prevent the enzymatic degradation of the DNA molecules inside the buffer. The labelling of the DNA molecules is done with the intercalating dye YOYO-1 (Invitrogen). In figure A2-5A the chemical structure of the dye is presented, whereas in B the analogue TOTO-1 intercalated with between the base pairs of a DNA molecule is presented. The YOYO-1 dye is very stable and is often used in single molecule studies and gel electrophoresis. Another advantage of this dye is the enhancement of the intensity of fluorescence once it is bound to DNA in this way the fluorescent background of the dye is very low. In the investigation of Larsson et $\mathrm{al}^{2}$. it was shown that the emission from YOYO-1 will be roughly perpendicular to the helical axis of the DNA molecule. Important to mention is that this intercalating dye is increasing the contour length of the DNA ${ }^{3}$ molecule and is also affecting the electrostatic properties of the DNA molecule. YOYO-1 has a charge of $4^{+}$and decreases the negative charge per unit length of the DNA when it is intercalated. This will increase the persistence length and the effective width of the 


\section{Single DNA Molecule Experiments}

molecule ${ }^{3}$. When working with fluorescent labels as YOYO-1, the loss of intensity of the signal as a result of photo-bleaching or photo-nicking remains a problem. Oxygen and hydroxyl radicals play an important role in this. To limit the presence of the free radicals in the buffer solution, one can degas the buffer solutions prior to use and add the antioxidant $\beta$-mercapto-ethanol ${ }^{4}$. The last chemical composition added to our buffer solution is polyvinylpyrrolidone (PVP). This is a polar polymer and is used to increase the viscosity in the electrical double layers close to the wall of the nanoslits. In this way, the electro osmotic flow is suppressed. Our nanoslit device is made of fused silica. With our experimental conditions, the surface charge of the nanoslits is negative. The DNA molecules are also negatively charged. Without the use of the PVP, we were not able to pull the $\lambda$-DNA molecules through the nanoslits by the applied electrical fields in DC.

Returning to the experimental conditions. First of all, it is important to mention that when working with biological samples as DNA molecules, on should use gloves and sterile pipette tips, tubes and glassware. Each contamination can influence the experiment. For example, on our skin, enzymes are present which can breakdown the DNA molecules.

The $\lambda$-DNA and XbaI digested Litmus DNA molecules (New England Biolabs) are stained with the fluorescent intercalating dye YOYO-1 (Invitrogen) at a ratio of 1 intercalating dye per 5 base pairs ${ }^{5}$. These will extent the contour length for the $\lambda$-DNA molecules to $\sim 21$ $\mu \mathrm{m}$ and for the Litmus DNA molecules to $\sim 1.2 \mu \mathrm{m}$. The staining was done by adding both DNA (final concentration $400 \mathrm{ng} / \mathrm{ml}$ ) and the YOYO-1 (final concentration $3 \mathrm{mM}$ ) to $1 \mathrm{x}$ Tris Borate Sodium EDTA (TBE) buffer ( $89 \mathrm{mM}$ Tris, $89 \mathrm{mM}$ Boric acid, $1 \mathrm{mM}$ EDTA, $\mathrm{pH}=8.3$ ). The solution was incubated for at least one hour at room temperature. Additionally, 2.5\% (w/w) polyvinylpyrrolidone (PVP) $(\mathrm{MW}=10000$ Dalton; Merck) to suppress electroosmotic flow and 3\% (v/v) $\beta$-mercaptoethanol to suppress photo-bleaching and photo-knicking were added ${ }^{3}$. Before the experiments, the solution was degassed in vacuum.

\section{A.2.6 References}

1. A. Pluen, B. Tinland, J. Sturm, G. Weill, 1998, Electrophoresis, 19, 1548-1559

2. W. Reisner, J. P. Beech, N. B. Larsen, H. Flyvbjerg, A. Kristensen, J. O. Tegenfeldt, 2007, Phys. Rev. Lett., 99, 058302.

3. B. Åkerman, E. Tuite, 1996, Nucleic Acid Research, 24, 1080-1090.

4. A. Larsson, C. Carlsson, M. Jonsson, B. Albinsson, J. Am. Chem. Soc. 1994, 116, 8459-8465.

5. T. Perkins D. Smith, R. Larson, and S. Chu, Science, 1995, 268, 83-87. 
Appendix 2 
Filename: Appendix 2_For_WPS_Final

Directory: $\quad$ C:Locuments and Settings/Georgette.UTWENTE-

A174A94\Desktop $\backslash$ Ready for printing $\backslash$ Dinsdag

Template:

C:LDocuments and SettingsiGeorgette.UTWENTE-

A174A94\Application DatalMicrosoft|Templates $\backslash$ Normal.dot

Title: 1

Subject:

Author:

Georgette

Keywords:

Comments:

Creation Date: $\quad$ 9/29/2009 11:44:00 AM

Change Number: 2

Last Saved On: $\quad$ 9/29/2009 11:44:00 AM

Last Saved By: Georgette

Total Editing Time: 0 Minutes

Last Printed On: $\quad$ 9/29/2009 11:44:00 AM

As of Last Complete Printing

Number of Pages: 8

Number of Words: $\quad 2,147$ (approx.)

Number of Characters: 10,135 (approx.) 


\section{Samenvatting}


In dit proefschrift wordt het onderzoek naar het electrokinetisch transport van (voornamelijk) lange DNA moleculen in nanokanalen beschreven. Deze nanokanalen zijn rechthoekig van vorm en zijn in de hoogte veel kleiner dan de breedte. De manier waarop deze lange DNA moleculen getransporteerd worden door deze nanostructuren is nog steeds niet helemaal begrepen. In dit proefschrift worden dan ook onverwachte resultaten beschreven.

De ontwikkeling van nanostructuren op chip opende de deur voor fundamenteel DNA onderzoek en voor nieuwe DNA scheidingstechnieken welke in de toekomst het gebruik van gelen zouden kunnen vervangen. Door het gebruik van geavanceerde nanotechnologieën kunnen gelen worden nagemaakt (bijvoorbeeld een array van nanopilaartjes of een nanofilter). Het voordeel van het gebruik van deze technieken is dat de structuren op maat gemaakt kunnen worden in geordende geometrieën. Dit in tegenstelling tot een gel, waarvan de poriën niet geordend zijn en waarvan de grootte beperkt wordt door de crosslinking en de mechanische eigenschappen van de gel.

In hoofdstuk 2 wordt een introductie gegeven van de belangrijkste theoriën welke ontwikkeld zijn voor "free-solution" en gel electroforese van DNA moleculen. Voorbeelden hiervan zijn het "extended Ogston model", "biased reptation" en "entropic trapping". Vanuit dit perspectief worden suggesties voor toekomstige concepten gegeven voor het gebruik van nanostructuren voor de snelle scheiding van DNA moleculen. Tevens wordt een deel van de besproken theoriën gebruikt om de resultaten uit dit proefschrift te verklaren.

In hoofdstuk 3 presenteren we het onderzoek naar het electrokinetisch transport gedrag van $\lambda$-DNA moleculen in $20 \mathrm{~nm}$ hoge nanokanalen gemaakt van siliciumoxide. De toegepaste electrische DC velden variëerden in sterkte van $4-200 \mathrm{kV} / \mathrm{m}$. We zien dat de mobiliteit van de moleculen van zowel het electrische DC veld als de lengte afhankelijk is. Tevens wordt een verschil in transport gedrag waargenomen tussen de lage en hoge electrische DC velden. In de hoge electrische DC velden kan de beweging van de DNA moleculen beschreven worden als een "Stop \& Go" beweging. De moleculen kunnen niet in een vloeiende beweging door de nanokanalen getransporteerd worden, maar stoppen af en toe. Dit in tegenstelling tot de electrische DC velden van $30 \mathrm{kV} / \mathrm{m}$ of lager waar de DNA moleculen getransporteerd worden in één vloeiende beweging. Tevens zien we een toename in mobiliteit to electrische DC velden van $30 \mathrm{kV} / \mathrm{m}$, wat verklaard zou kunnen worden met behulp van het "biased reptation" model. Zolang het molecuul nog niet volledig gericht is in de richting van het electrische DC veld, kan het in snelheid (en dus mobiliteit) toenemen met toenemende electrisch DC veldsterkte. De aanwezigheid van voorkeurs routes van de $\lambda$-DNA moleculen in de nanokanalen ondersteunen dit nog eens extra. Een vergelijking van de mobiliteiten van $\lambda$-DNA and Litmus DNA moleculen liet een dusdanig verschil zien wat veelbelovend is voor het gebruik van deze nanokanalen om DNA moleculen electroforetisch te scheiden.

In hoofdstuk 4 wordt het verdere onderzoek naar het onstaan van de onderbroken beweging van de $\lambda$-DNA moleculen bij hoge electrische DC velden beschreven. Als eerste werd er 
gekeken naar de mogelijke aanwezigheid van dielectroforetische hindernissen in de $20 \mathrm{~nm}$ hoge nanokanalen. Dit werd gedaan door electrische AC velden $(10-200 \mathrm{kV} / \mathrm{m})$ met frequenties van $100 \mathrm{~Hz}$ of $1 \mathrm{kHz}$ toe te voegen aan electrische DC velden van $8-200$ $\mathrm{kV} / \mathrm{m}$ en het electrokinetisch transport gedrag van $\lambda$-DNA te onderzoeken. In alle onderzochte electrische DC velden lager dan $20 \mathrm{kV} / \mathrm{m}$ en toegevoegde electrische AC velden $(1 \mathrm{kHz})$ werd een significante toename in mobiliteit gevonden wat niet verwacht werd indien er dielectroforetische hindernissen aanwezig zouden zijn. De toename in mobiliteit kan ook hier mogelijk verklaard worden met een toename in de oriëntatie van het molecuul in de richting van het electrisch DC veld en is dus in overeenstemming met het "biased reptation model". Dit is dan vergelijkbaar met resultaten van onderzoeken verkregen met gel electroforese. De gemeten mobiliteiten in electrische DC velden hoger dan $20 \mathrm{kV} / \mathrm{m}(100 \mathrm{~Hz}$ en $1 \mathrm{kHz})$ geven op een paar uitzonderingen na (welke verder onderzocht moeten worden) een complex beeld en geen bewijs voor de aanwezigheid van dielectroforetische hindernissen.

In hoofdstuk 5 beschrijf ik de metingen aan de mobiliteit van $\lambda$-DNA moleculen in nanokanalen van 60 en $120 \mathrm{~nm}$ hoog en worden de resultaten vergeleken met die van de 20 $\mathrm{nm}$ hoge nanokanalen. Interessant is dat de mobiliteit tussen $15-40 \mathrm{kV} / \mathrm{m}$ afneemt met de hoogte van het nanokanaal $(20 \mathrm{~nm}>60 \mathrm{~nm}>120 \mathrm{~nm})$ en dat dit terug gevonden wordt in eerste computer simulaties. Ook in deze nanokanalen is gekeken naar de aanwezigheid van de onderbroken bewegingen van de $\lambda$-DNA moleculen bij de hoge electrische DC velden. Dit is alleen in de $60 \mathrm{~nm}$ hoge nanokanalen terug gevonden. Dus de aanwezigheid van een mechanish (en/of ander) soort hindernis bij hoge electrische DC velden (>40 kV/m) neemt toe met afnemende slit hoogte. De afwezigheid van voorkeur routes van de $\lambda$-DNA moleculen zoals gevonden in de $20 \mathrm{~nm}$ hoge nanokanalen, samen met de grofweg constante mobiliteit bij alle toegepaste electrische DC velden, geeft an dat de $\lambda$-DNA moleculen zich hier niet in het "biased reptation" regime bevinden.

In het laatste hoofdstuk beschrijf ik de productie van een nieuw ontworpen nanokanaal. Aan één kant van dit nanokanaal bevindt zich een lijnen patroon welke samengesteld is uit $\mathrm{SiO}_{2}$ en $\mathrm{Al}_{2} \mathrm{O}_{3}$. Onder de juiste omstandigheden zal dit patroon een lading hebben. Dit lijnen patroon is onder een bepaalde hoek in de lengte van het nanokanaal gemaakt. Er wordt verwacht dat de scheiding van biomoleculen mogelijk is als het nanokanaal klein genoeg is (in hoogte) en dus de Coulomb interactie tussen patronen oppervlak en biomolecuul sterk genoeg is. De dimensies van patronen zijn gekozen naar aanleiding van de grenzen in de fotolithografie en gemaakt in Borofloat wafers. Om een glad oppervlak te krijgen werden de wafers chemisch-mechanisch gepolijst. In een andere Borofloat wafer werden alle microkanalen en het nanokanaal gemaakt. Na anodische bonding werden de wafers gezaagd. In een eerste experiment kon het nanokanaal gevuld worden met $0.1 \mathrm{mM}$ $\mathrm{NaAc}$ buffer $(\mathrm{pH}=5)$.

Ter conclusie, we hebben een relatief eenvoudige nanokanalen chip gebruikt om de electrokinetische transport eigenschappen van $\lambda$-DNA moleculen te bestuderen. Het experimentele werk gepresenteerd in dit proefschrift draagt bij an het beter begrijpen van 
de regimes en theoriën als kort beschreven in hoofdstuk 2. De verschillen in mobiliteit die we vonden tussen $\lambda$-DNA en Litmus DNA moleculen zijn veelbelovend voor de ontwikkeling van nieuwe scheidings apparatuur. Los van dit alles hebben we ook een nieuwe nanokanalen chip geproduceerd welke aan één kant van het nanokanaal een ladings patronen oppervlak bevat. We hopen hiermee in de toekomst spannende metingen te doen!

Georgette B. Salieb-Beugelaar

Oktober 2009 
Filename: Samenvatting_For_WPS_Final_Reserve

Directory: $\quad$ C: $\quad$ Documents and Settings\Georgette.UTWENTE-

A174A94\Desktop\Ready for printing $\backslash$ Reserve

Template:

C:IDocuments and Settings\Georgette.UTWENTE-

A174A94\Application DatalMicrosoft $\backslash$ Templates $\backslash$ Normal.dot

Title:

Summary

Subject:

Author:

Georgette

Keywords:

Comments:

Creation Date: $\quad$ 9/29/2009 9:08:00 PM

Change Number: 2

Last Saved On: $\quad$ 9/29/2009 9:08:00 PM

Last Saved By: Georgette

Total Editing Time: 1 Minute

Last Printed On: $\quad$ 9/29/2009 9:08:00 PM

As of Last Complete Printing

Number of Pages: 4

Number of Words: $\quad 1,068$ (approx.)

Number of Characters: $\quad$ 6,089 (approx.) 


\section{Curriculum Vitae}




\section{Qualifications:}

1989-1995. B.Sc. Biomedical Chemistry. HTO IJselland (Deventer - The Netherlands). Thesis: Mutation Detection in Patients with Cerebrotendinous Xanthomatosis.

2000-2003. M.Sc. in Chemistry. University of Utrecht (Utrecht-The Netherlands). Thesis: Structural Elements of the Bacterial Potassium Channel KcsaI; a bioinformatical and experimental study.

Subthesis: The Enzymatic Deacylation and Reacylation of Bacterial Membrane Lipids.

2005-2009, PhD student, University of Twente (Enschede - The Netherlands)

PhD thesis: "Electrokinetic transport of DNA in nanoslits"

\section{Employment history:}

7/1994-7/1995. Academic Hospital St. Radboud (Nijmegen - The Netherlands). Department of Neurology and Children Medicine, Laboratory of DNA diagnostics. Thesis (B.Sc.): Mutation Detection of Patients with Cerebrotendonous Xanthomathosis.

1/1996-11/2005. Academical Medical Centre (Amsterdam - The Netherlands). Department of Clinical Genetics, Laboratory of DNA diagnostics \& research. 1996-2003: 50\% diagnostic and 50\% research technician.

2004-2005: 10\% diagnostic, 90\% research in the epigenetics of the Beckwith Wiedemann syndrome.

8/2001-7/2002. University of Utrecht, Faculty of Chemistry (Utrecht - The Netherlands). Department of Biochemistry of Membranes.

Thesis (M.Sc.): Structural Elements of the Bacterial Potassium Channel KcsA - a bioinformatical and experimental study.

11/2005 -10/2009. University of Twente, Faculty of Electrical Engineering, Mathematics and Computer Science /Mesa ${ }^{+}$Institute for Nanotechnology (Enschede - The Netherlands).

PhD Student at the department of Biosensors (BIOS Lab-on-a-Chip group) / Mesa ${ }^{+}$ Institute for Nanotechnology.

8/2009-10/2009, University of Twente, Faculty of Electrical Engineering, Mathematics and Computer Science (Enschede - The Netherlands) / FRIAS - Freiburg Institute for Advanced Studies, School of Soft Matter Research, Albert-Ludwigs-Universität Freiburg (Germany)

HPLC on chip project. 


\section{Curriculum Vitae}

11/2009-......... Korean Institute of Science and Technology Europe, Forschungsgesellschaft mbH - Saarbrücken (Germany)

\section{Courses:}

2004. Introduction to Bioinformatics (Science Education for PhD Students, Academic Medical Centre, Amsterdam - The Netherlands).

Subjects of the course were: databases, basecalling and assembly, statistics, sequence alignment, gene finding, image analysis, DNA microarray analysis, SAGE, scoring matrices, phylogenetics and proteomics.

2006. MESA Cleanroom courses (University of Twente, Enschede - The Netherlands). Introduction course, Chemical and safety course, MTE workshop (standard wafer cleaning, oxidation and diffusion, annealing, photolithography, mask stripping).

2006. FSRM Training in Microsystems (Parc Cientific Barcelona, Barcelona - Spain). Labs-on-Chips Technologies: Basics and Applications.

Subjects of course were: basic microfluidic principles, fabrication/biotech background, classification of LOC systems, call-based systems, microfluidic systems for sample preparation/proteins/DNA, nanofluidics and surface based systems/commercialization.

2007. FSRM Training AFM (Online course).

Microcantilever Applications

Introduction to possible applications of microcantilever technology as sensors and actuators in high growth markets (Biomedical, Security and Environmental) and other industries.

2007. Technical Writing (University of Twente, Enschede - The Netherlands).

Course in technical writing.

2008, How to speak in front of an audience (Champery - Swiss, 9-12/01/08)

2008, Label Free Biosensing (University of Twente, Enschede - The Netherlands)

Introduction course in plasmon resonance techniques. 
Filename: $\quad$ Curriculum Vitae_For_WPS_Final_Reserve

Directory: $\quad$ C:Locuments and SettingslGeorgette.UTWENTE-

A174A94\Desktop\Ready for printing $\backslash$ Reserve

Template:

C:Documents and SettingslGeorgette.UTWENTE-

A174A94\Application DatalMicrosoft|Templates $\backslash$ Normal.dot

Title:

Curriculum Vitae

Subject:

Author:

Keywords:

Comments:

Creation Date: $\quad$ 9/29/2009 11:12:00 AM

Change Number: 2

Last Saved On: $\quad$ 9/29/2009 11:12:00 AM

Last Saved By: Georgette

Total Editing Time: 1 Minute

Last Printed On: $\quad$ 9/29/2009 11:12:00 AM

As of Last Complete Printing

Number of Pages: 4

Number of Words: $\quad 650$ (approx.)

Number of Characters: 3,071 (approx.) 


\section{List of publications}




\section{Publications:}

Salieb-Beugelaar G.B. , Czerwinska J., van Nieuwkasteele J.W., Wijnperlé D., van den Berg A., Eijkel J.C.T. Dynamical conformation and mobility of $\lambda$-DNA in confined nanoslit environment. In preparation

Castillo-Fernandez O, Salieb-Beugelaar GB, van Nieuwkasteele J.W., Bomer J.G., Arundell M., Samitier J., van den Berg A., Eijkel J.C.T. AC/DC field dependent DNA mobility in $20 \mathrm{~nm}$ high nanoslits. In preparation

Salieb-Beugelaar GB, Castillo-Fernandez O, van Nieuwkasteele J.W., Bomer J.G., Arundell M., Samitier J., van den Berg A., Eijkel J.C.T. DNA transport in $20 \mathrm{~nm}$ nanoslits by AC and DC electrical fields. The proceedings of microTAS 2009 Conference, 1-5 November 2009, Jeju, Korea. Micro Total Analysis Systems. Chemical and Biological Microsystems Society.

Salieb-Beugelaar GB, Dorfman KD, van den Berg A, Eijkel JCT (2009). Electrophoretic Separation of DNA in Gels and Nanostructures. Lab on a Chip, DOI:10.1039/B905448K.

Salieb-Beugelaar GB, Teapal J, van Nieuwkasteele JW, Wijnperlee D., Tegenfeldt JO, Lisdat F., van den Berg A., Eijkel JCT (2008). Field Dependent DNA Mobility in $20 \mathrm{~nm}$ High Nanoslits. Nano Letters 8, 7: 1785-1790.

Awarded: Best publication 2008 Nano2Life.

Salieb-Beugelaar GB, Teapal J., van Nieuwkasteele JW, Wijnperle D., Tegenfeldt J.O., Eijkel J.C.T. and van den Berg A. (2007). DNA movement in sub-20 nm nanoslits. In: Eleventh International Conference on Miniaturized Systems for Chemistry and Life Sciences - The proceedings of microTAS 2007 Conference, 7-11 october 2007, Paris. pp. 1201-1203. Micro Total Analysis Systems 2. Chemical and Biological Microsystems Society. ISSN 1556-5890 ISBN 978-0-9798064-0-7

Bliek J, Terhal P, van den Bogaard MJ, Maas S, Hamel B, Salieb-Beugelaar GB, Simon M, Letteboer T, van der Smagt, Kroes H, Mannens MM (2006). Hypomethylation of the H19 gene causes not only Silver-Russell Syndrome (SRS) but also isolated asymmetry or an SRS-like phenotype. The American Journal of Human Genetics 78: 604-614.

Breugem CC, Alders M, Salieb-Beugelaar GB, Mannens MMAM (2002). A locus for hereditary capillary malformations mapped on chromosome 5q. Human Genetics 110: 343347.

Aalfs CM, Salieb-Beugelaar GB, Wanders RJ, Mannens MM, Wijburg FA (2000). A case of methemoglobinemia type II due to NADH-cytochrome b5 reductase deficiency: determination and molecular basis. Human Mutation 16: 18-22. 
List of publications

\section{Contributed to DNA work of:}

de Lange J, van Maarle MC, van den Akker, HP, Redeker EJW (2007). A new mutation in the SH3BP2 gene showing reduced penetrance in a family affected with cherubism. Oral Surgery, Oral Medicine, Oral Pathology, Oral Radiology, and Endodontology, 103, 3: 378381.

Bezzina CR, Rook MB, Groenewegen WA, Herftst LJ, van der Wal AC, Lam J, Jongsma HJ, Wilde AA, Mannens MM (2003). Compound heterozygosity for mutations (W156X and $\mathrm{R} 225 \mathrm{~W}$ ) in SCN5A associated with severe cardiac disturbances and degenerative changes in the conduction system. The American Journal of Human Genetics 73: 13851401 .

Alders M, Ryan A, Hodges M, Bliek J, Feinberg AP, Privitera O, Westerveld A, Little PFR, Mannens MMAM (2000). Disruption of a Novel Imprinted Zinc-Finger Gene, ZNF215, in Beckwith-Wiedemann Syndrome. Am. J. Hum. Gen. 66, 5: 1473-1484.

\section{Other contributions (abstracts, posters and invitations):}

NanoNed 2006 (Utrecht, The Netherlands), poster presentation. Title: Influence of Ionic Strength on DNA Transport Through 5 and 12 nm High Channels.

Nanotech 2006 (Montreux, Switzerland), poster presentation. Title: Influence of Ionic Strength on DNA Transport Through 5 and 12 nm High Channels.

NanoMicroNed 2006 (Eindhoven, The Netherlands), poster presentation. Title: Influence of Ionic Strength on DNA Transport Through 5 and $12 \mathrm{~nm}$ High Channels.

NanoNed 2007 (Boekelo, The Netherlands), oral presentation. Title: Molecular Separation in Nanofluidic Devices.

NanoBio 2007 (Münster, Germany), invited for oral presentation. Title: Mobililty of DNA in Sub $20 \mathrm{~nm}$ Nanoslits.

Mesaday 2007 (Enschede, The Netherlands), oral presentation. Title: Mobililty of DNA in Sub 20 nm Nanoslits.

MicroTas 2007 (Paris, France), oral presentation. Title: Mobililty of DNA in Sub $20 \mathrm{~nm}$ Nanoslits.

Nano2Life 2007 (Lund, Sweden), invited for an oral presentation. Title: DNA mobility through sub $20 \mathrm{~nm}$ nanoslits. 
NanoNed 2007 (Nijmegen, The Netherlands), poster presentation. Title: Field dependent DNA mobility in $20 \mathrm{~nm}$ high nanoslits.

Nano-MicroNed 2007 (Nijmegen, The Netherlands), oral presentation. Title: Field dependent DNA mobility in $20 \mathrm{~nm}$ high nanoslits.

Annual Meeting Nano2Life 2008 (Champery, Switzerland), poster presentation. Title: Field dependent DNA mobility in $20 \mathrm{~nm}$ high nanoslits.

Awarded: third prize best young scientist poster Nano2Life.

Annual Meeting Nano2Life 2008 (Champery, Switzerland), poster presentation. Title: Nanopatterning of a nanoslit device.

University of Twente - University of Münster meeting 2008, poster presentation. Title: Field dependent DNA mobility in $20 \mathrm{~nm}$ high nanoslits.

NanoNed 2008 (Boekelo, The Netherands), oral presentation. Titles: Field dependent DNA mobility in $20 \mathrm{~nm}$ high nanoslits \& The nanopatterning of a nanoslit device.

Nano2Life 2008 (Heraklion, Greece), oral presentation. Title: DNA, nanoslits and electrical fields.

Nano2Life 2008 (Heraklion, Greece), poster presentation. Title: DNA, nanoslits and electrical fields.

Nano-MicroNed 2008 (Ede-Wageningen, The Netherlands), poster presentation. Title: $\mathrm{AC} / \mathrm{DC}$ electrical fields and the mobility of DNA in nanoslits.

Nanotech 2008 (Montreux, Switzerland), oral presentation. Title: AC/DC electrical fields and the mobility of DNA in nanoslits.

Najaars symposium KNCV / WGS 2008 (Amsterdam, The Netherlands), poster presentation. Title: Field dependent DNA mobility in $20 \mathrm{~nm}$ high nanoslits.

NanoNed 2008 (Utrecht, The Netherlands), poster presentation. Title: AC/DC field dependent DNA mobility in $20 \mathrm{~nm}$ high nanoslits

MicroTas 2009 (Jeju, Korea), poster presentation. Title: DNA transport in $20 \mathrm{~nm}$ nanoslits by AC and DC electrical fields.

NanoTech 2009 (Montreux, Switzerland), poster presentation. Title: DNA in nanoslits; trapping and biased reptation.

Micro/NanoNed 2009 (Delft, The Netherlands), oral presentation. Title: DNA in nanoslits; trapping and biased reptation. 
Filename: $\quad$ New_List of publications_For_WPS_Final

Directory: $\quad$ C:LDocuments and Settings/Georgette.UTWENTE-

A174A94lDesktop

Template:

C:IDocuments and Settings $\backslash$ Georgette.UTWENTE-

A174A94\Application DatalMicrosoft|Templates $\backslash$ Normal.dot

Title:

Curriculum Vitae

Subject:

Author:

Georgette

Keywords:

Comments:

Creation Date: $\quad$ 10/8/2009 11:05:00 AM

Change Number: 2

Last Saved On: $\quad$ 10/8/2009 11:05:00 AM

Last Saved By: Georgette

Total Editing Time: 0 Minutes

Last Printed On: $\quad$ 10/8/2009 11:05:00 AM

As of Last Complete Printing

Number of Pages: 4

Number of Words: 952 (approx.)

Number of Characters: $\quad 5,427$ (approx.) 


\section{Acknowledgements/ Dankwoord}


Het laatste deel van mijn proefschrift is nu gekomen. Het schrijven van het dankwoord. Ik heb geen idee waar ik moet beginnen en wie ik als eerste moet bedanken. Daarom begin ik maar gewoon met schrijven... De jaren zijn voorbij gevlogen. Het verhaal in Enschede begon eigenlijk bij Istvan, wie ik dankbaar ben voor de fijne gesprekken sindsdien. Ik denk nog wel eens terug hoe anders alles had kunnen zijn. Maar aangezien ik altijd zoiets heb van "je krijgt toch wel wat voor je weg gelegd is..." ben ik zeer tevreden met zoals alles gegaan is. En zo kwam ik bij Jan en Albert terecht. Vooral het eerste jaar vond ik helemaal geweldig. Ik heb heel veel plezier met jullie gehad!! Dat heeft me ook buitengewoon gemotiveerd om dit allemaal te doen. Het is echt een wereld van verschil tussen het AMC en Twente University, maar ik heb nooit 1 seconde spijt gehad dat ik van richting ben veranderd. Eén van de dingen die me bij zal blijven zijn onze werkoverleggen Jan \& Albert! Wat heb ik vaak gelachen om jullie tegenstellingen. Dan zat ik aan tafel te wachten, terwijl jullie heel heftig aan het discussiëren waren. Bedankt voor de fijne tijd en vooral ook steun die jullie me gegeven hebben. Ik heb waanzinnig veel van jullie geleerd!! Vervolgens Hermine, ik vind het heerlijk om je kamer binnen te rennen en gelijk mijn hart bij je uit te storten. Maar dat zul je inmiddels wel weten. Ik vind het ook zalig om samen met jou Albert voor de gek te houden. Ook jij bent inmiddels veel meer dan een gewone collega voor me geworden. Hopelijk groeit onze vriendschap nog heeeel lang door!!!! Ik heb ontzettend genoten van onze lunchen samen met lieve Wies. Wies, jij bedankt voor de interesse in mijn werk en prive en de gezelligheid die je altijd meebrengt. Ik heb bewondering voor je! Bovenaan dit lijstje staat ook Johan. Toen ik de knoop doorhakte om met het imprintwerk van onze structuren te stoppen, was jij degene die me nog motiveerde om nog 1 ding te proberen. Uiteindelijk heb je het toch voor elkaar gekregen om een patronen chip the produceren. Petje af! Waar zouden we zijn zonder technici! Bedankt voor de fijne tijd samen, je droge humor en je kundigheid in Clean Room! Ah ja, en nu mijn paddo-maatje: @d. Bedankt Ed, voor alle wandelingen, heerlijke gesprekken, skeeler avonturen en alle andere gekke momenten (teken, gevaarlijke babyvogeltjes etc). Waar mijn levenspad ook heen loopt, ik zal je blijven klieren! De BIOS groep is ook geweldig. Vooral onze reis naar Denemarken zal ik nooit vergeten. Floor, Eric F. (mijn overgetelijke kamergenoot! Wanneer gaan we lunchen?) Erik K., Sebastiaan, Roald, Lee, Ana, Jurjen (salmiakjesss) en alle anderen bedankt voor de waanzinnige tijd samen daar! Vooral de kampvuren tot diep in de nacht blijven me bij. De samenstelling van de BIOS groep is inmiddels wel veranderd, maar het blijft een heerlijke groep. Hoewel mijn toekomst nog niet helemaal vast ligt, is het bijna zeker dat ik jullie ga verlaten. Ik moet verder, ik ben en blijf een zwerver. Dus kamergenootjes..... Songyue en Evelien, ik zal jullie missen! Sturen jullie een update van de Chinese lessen af en toe? Dan wil ik Wouter en Lingling bedanken. Wouter, ik heb veel van je geleerd en vond het fijn om met jou de microscoop te delen! Je weet een hoop en als ik ergens niet uit kwam, stond je altijd klaar om me te helpen! Bedankt! Ergens zal ik dit donkere rommelhok (laserlab) wel missen. Lingling, it is very nice to work with you, and I am so happy that we will continue this at least for a few months! Vervolgens, Jan van Nieuwkasteele, ik zal de Mac gesprekken missen! Het spijt me dat ik je prachtige gekleurde wafers niet meer in een mosaic heb verwerkt voor de cover. Ik zat "gevangen" in de bergen met mijn scheurijzertje.... Maar, ik ga het kunstwerk alsnog maken. Je kunt dus tzt een foto ervan verwachten. En Daniël, naast de gezellige gesprekken stond je ook altijd voor me klaar. Ik ben heel erg blij dat jullie nu een kindje 
hebben! Geniet ervan, ze zijn zo groot. Verder, Iris, Ganesh, Arpita, Mingliang, Muhammad, Maarten, Paul, Loes, Mathieu, Eddy, Hans, Edwin, Ad, Wouter O., Egbert, Severine, (en nu buiten BIOS) Svetlana, AFM-Martin, Cabrio-Thijs, Microscoop-Henk, Cleanroom-Ton en Huib, Nanoimprint-Boris (take care, I will not be that far from Aachen!), Electronenmicroscoop-Mark, Mesa-Miriam, Bianca, Jacob, Wojtek, Wim en alle andere mensen die ik vergeten ben op te noemen: bedankt voor de onvergetelijke tijd!

Het thuishonk AMC kon ik ergens niet loslaten. Dit had ik nooit gedacht. Natuurlijk één van de hoofdoorzaken is Kaatje. In voor en tegenspoed, ben je voor mij alweer 13 een belangrijk persoon in mijn leven. Bedankt girlie, vooral voor het aanhoren van alle belevenissen en ontwikkelingen in vooral het eerste jaar. Enne, leuk die tukker (Koenie) van je! Wees er zuinig op! Dan is er natuurlijk Jet. Geweldig zoals je proefschrift geworden is! Marcel, jij ontzettend bedankt voor je gastvrijheid. Weet je nog wat ik ooit tegen je zei? Ik wil promoveren. Nou, voilà! Bij deze $\odot$ ! Maar ook mijn andere collegae als Sebastiaan, Zahir, Dennis, Maureen, Loraine, Ruud, Mariel, Bert, Marielle, Aho, Tamara, Leander, Alex en alle anderen die vergeten ben, bedankt voor het vormen van een soort thuisfront. Zo nu en dan kwam ik binnen rennen om DNAtjes te scoren of rommel te lenen (gelbakken), en ik moet toegeven, dat voelde heel erg fijn! Mochten jullie de gelbak niet meer terug willen, neem ik hem mee naar mijn volgende plekkie hehe... Maar, het AMC verhaal is nog niet ten einde. Er is nog een heel belangrijk iemand voor me. Omdat ik altijd weer opzoek ga naar nieuwe uitdagingen ging ik met enige regelmaat met Guus kletsen. Guus, jouw lab is natuurlijk een buitengewoon interessante speelplaats voor mensen zoals ik. Bedankt voor de heerlijke gesprekken over wetenschap, àlle andere diepgaande conversaties maar vooral ook voor de steun in mijn laatste jaar. EN: Ik zei je toch ooit: nieuwe ideeën worden op de gekste momenten geboren? Dit betekent dus ook dat je voorlopig nog niet van me af bent. Ik loop nog steeds met exosomen in mijn hoofd. Zeg dat maar tegen Rienk! Trouwens, Rienk, jij óók bedankt voor je interessante vertellen! Niet alleen over exosomen en microparticles, maar ook over vogels. Ik ben gek van allerlei soorten vogeltjes.

Voor mijn buitenlandse vrienden en collegae, schakel ik nu even over naar het engels. Last four years I met and worked with many people. Some of them became real friends. I really need to thank a few people in particular. First of all Jack, you have really no idea how much you helped me through the difficult times. For example the time that we were studying together while keeping in touch via Skype was a great experience! I also enjoyed very much our time together when we visited musea (Pola is still remembering the China Exhibition!) or explored foreign countries (in the snow (-))! Amazing that we never got lost! Of course I need to thank Torsten and Moshe for the marvelous time we had during our Nano2Life (N2L) meetings. It was very nice to go to a meeting knowing you both would be there. You remember Torsten our last N2L meeting without Moshe? Something was really missing..... Also the meeting when Moshes' friend Koby joined was so exceptional. Thank you Koby, for making the movie with all the nice moments in Champery! Of course I hope we will make more memories together, like the day in Amsterdam we had in February this year. Even tussendoor...Tsafira, bedankt voor het vertalen van een tekst naar het Hebreeuws voor mijn vrienden. Het meegestuurd met het proefschrift. Continuing with N2L, the next person is Eric Moore. Yes, you are also on my 
list. Do you remember from a long time ago the "cause and effect", discussion? It was in Münster during a conference dinner. But also the years after, I enjoyed the fun of the "mobility sessions". Thank you so much for the nice time we had. Than there are three very sweet N2L ladies which are also special to me. I would like to thank them for being there during meetings and for the nice correspondense always in between: Hildegard, Sabine and Jenny. The last meeting really impressed me, it was so incredibly sad to say goodbye. Of course there were many other nice N2L people as Ingolf, Partick, Patrice, Dieter, Lars and others who are not mentioned here.

Andreas, of course you are in my list as well! The spinning car-fun gave me really huge adrenalin shots. To be honest, I do dream now of becoming a Formula-1 driver (in the mountains). Of course I am thankful for all other special moments and conversations and most important: just being there for me. To be continued in Saarbrücken!

During the last 4 years I had several fruitful collaborations. The first one was with Jonas Tegenfeldt who gave Juliane Teapal an introduction in the "DNA in nanoslit" world. She transferred this knowledge successfully to Twente. I would like to thank both Jonas (and co-workers on the lab like Jason!!) as Juliane for the nice time we had. I hope to visit you, Christelle and the kids one day Jonas! Of course when I go there I will find you as well Dr. Persson!!! We should at least drink a nice wine and continue the "Hasta la macaroni"-fun. I really enjoyed our crazy conversations in the middle of the writing stress! Tack så mycket!! The collaboration with Oscar was also great! It was very interesting to see how you transformed the setup to do the AC measurements. I had fun in Barcelona those days in May earlier this year. It was very good to see the Spanish way of working, which is completely different to my way. Muchas gracias! The last collaboration was with Justyna. We haven't seen each other that much, however it was impressive. When you were in Twente, using the SARA computer in Amsterdam, we met from time to time. Showing me 3D movies of moving DNA molecules in nanoslits. After this, we started to mail and sms and in a way a friendship is growing. Hopefully to be continued! See you soon? Somewhere in between Poland and the Netherlands?

Next to this there were also students. Xavi, it was fun to have a PhD student as a student. I hope you had a great time in Twente and that you learned a lot from the (nano-)imprinting in our cleanroom. Another very nice student was Caroline. She managed to write a plug-in for ImageJ which I could use for the data analysis of the DNA movies. I still would love to spend more time in using this, especially while now the quality of the movies is very improved. Hopefully we will meet one day in the south of France. May be next summer. Than I would love to visit Santander (the north of Spain), my friends there and next to this, to remember my sweet "abuelita Isabel". Isabel, you are still there, very very deep in my heart, as I always told you...... Thank you so much for being there, ever since I was a little girl.

Tja, ik ben nu inmiddels aangekomen bij het allerlaatste gedeelte, mijn familie. Ik begin bij mijn lieve ouders. Paps, bedankt voor alle motivatie en de heerlijke gesprekken over het onderzoek. Je was een echte steun voor me. Mams (hoewel het laatste jaar wel heel erg chaotisch was) ik genoot altijd van onze dagjes weg. Dat moeten we nu maar weer eens gauw gaan doen! Lekker bijkletsen, winkelen, antiek zilver zoeken... Mijn broer Roy, in vele opzichten ben je net zo gek als ik. Dat is altijd een fijn gevoel geweest, dan ben ik tenminste niet de enige! Ik zie er naar uit om samen met Jen \& Kinayro en mijn gezin een 


\section{Acknowledgements / Dankwoord}

weekend weg te gaan. Eindelijk tijd om fossielen te zoeken! Naast dit verheug ik me ook om nog eens op vakantie te gaan met Sarwat, Lucy, Samira, Marco, Ramona, Dimyana, Jamila! Alleen nu moeten we maar eens naar de sneeuw gaan! Daar zullen we ook vast wel een hoop plezier hebben. Ik heb nog wel wat ideetjes! Bedankt dat jullie er altijd voor me waren. Of course I have also family in Egypt. First of all, I would like to thank Baba for always supporting me in my work. Especially last months. I really hope to see you soon. Next to this, I really missed all the other family members. I do have so many precious moments in my mind ever since 1996. Unfortunately, last years we did not see each other that much anymore. Hopefully we will meet soon again.

In het bijzonder en bovenaan iedereen wil ik Maher en Pola mijn fute-kind bedanken. Maher, je bent in alle opzichten een topvent! Ik ben je vanuit diep in mijn hart dankbaar voor de mogelijkheden die je me gegeven hebt. Ik weet dat dit soms heel erg moeilijk is geweest en daarom bewonder ik je voor je doorzettingsvermogen! Ze zeggen altijd: $\mathrm{Na}$ regen komt zonneschijn! Dus..... En Pola, weet je nog, dat fute-kind in het meer van Locarno? Prachtig toch, zo'n dwingend kuikentje? Jij bent mijn fute-kind. Dit heb ik voor je vertaald naar Grebe (fuut) son (zoon), Grebeson Art (zie bladzijde 2 onderaan). Jouw getekende DNA moleculen op de cover van dit proefschrift, geplakt bovenop een andere kostbare herinnering.....

En nu....tijd voor iets anders.....Black Devil....waar ben je?

Georgette 
Filename: Acknowledgements_WPS_Final

Directory: $\quad$ C:Documents and SettingsiGeorgette.UTWENTEA174A94lDesktoplDinsdag

Template:

C:Documents and SettingsiGeorgette.UTWENTE-

A174A94\Application DatalMicrosoft|Templates $\backslash$ Normal.dot

Title: Indeling proefschift:

Subject:

Author:

Keywords:

Comments:

Creation Date: $\quad$ 9/30/2009 6:36:00 AM

Change Number: 5

Last Saved On: $\quad$ 9/30/2009 7:46:00 AM

Last Saved By: Georgette

Total Editing Time: 7 Minutes

Last Printed On: $\quad$ 9/30/2009 7:46:00 AM

As of Last Complete Printing

Number of Pages: 5

Number of Words: $\quad 1,955$ (approx.)

Number of Characters: 11,149 (approx.) 


\section{Stellingen}

1. Interdisciplinaire kennis is een verrijking van de geest.

(Hoofdstuk 3-6)

2. Single molecule studies blijven onverwachte resultaten geven. (Hoofdstuk 3-5)

3. Als je zaken achteraf bekijkt, lijkt een puzzel altijd makkelijker dan aan het begin.

(Hoofdstuk 3)

4. Bij electrische DC velden tussen $10-40 \mathrm{kV} / \mathrm{m}$ is de mobiliteit van een $\lambda$-DNA molecuul hoger naarmate het nanokanaal kleiner is $(20 \mathrm{~nm}$ $>60 \mathrm{~nm}>120 \mathrm{~nm}$ ). Hier is de frictie met de wand van het nanokanaal niet bepalend voor de mobiliteit van het DNA molecuul.

(Hoofdstuk 5)

5. De mens staat in grootte tussen het heelal, met zijn onvoorstelbare afstanden en reusachtige krachten, en de wereld van de kleinste deeltjes. Het is bijzonder dat juist de mens door zijn verstandelijk vermogen in staat is deze bereikbare wereld van het kleinste voor zich dienstbaar te maken.

6. Binnen een tiental jaren kan de eigenaar echt met zijn hond communiceren met behulp van de computer.

7. De electrische auto zal een aanzienlijk beter rijcomfort bieden.

8. Geluk is meer levenswijsheid dan mazzel.

9. Gentechnologie is een olifant in een porseleinkast.

10. De $11^{\mathrm{e}}$ stelling bij een proefschrift is de moeilijkste. 